\title{
REVIEW ARTICLE OPEN Structural biology of SARS-CoV-2: open the door for novel therapies
}

\author{
Weizhu Yan ${ }^{1}$, Yanhui Zheng ${ }^{1}$, Xiaotao Zeng ${ }^{1},{\text { Bin } \mathrm{He}^{2,3 凶} \text { and Wei Cheng }}^{1 凶}$
}

Severe Acute Respiratory Syndrome Coronavirus-2 (SARS-CoV-2) is the causative agent of the pandemic disease COVID-19, which is so far without efficacious treatment. The discovery of therapy reagents for treating COVID-19 are urgently needed, and the structures of the potential drug-target proteins in the viral life cycle are particularly important. SARS-CoV-2, a member of the Orthocoronavirinae subfamily containing the largest RNA genome, encodes 29 proteins including nonstructural, structural and accessory proteins which are involved in viral adsorption, entry and uncoating, nucleic acid replication and transcription, assembly and release, etc. These proteins individually act as a partner of the replication machinery or involved in forming the complexes with host cellular factors to participate in the essential physiological activities. This review summarizes the representative structures and typically potential therapy agents that target SARS-CoV-2 or some critical proteins for viral pathogenesis, providing insights into the mechanisms underlying viral infection, prevention of infection, and treatment. Indeed, these studies open the door for COVID therapies, leading to ways to prevent and treat COVID-19, especially, treatment of the disease caused by the viral variants are imperative.

Signal Transduction and Targeted Therapy (2022)7:26 ; https://doi.org/10.1038/s41392-022-00884-5

\section{INTRODUCTION}

SARS-CoV-2, a novel human coronavirus, broke out in December 2019 and has infected more than 230 million people and caused 4.87 million deaths, according to the latest data from World Health Organization (WHO; https://www.who.int/emergencies/diseases/ novel-coronavirus-2019). Coronaviruses (CoVs) have the largest genomes of the positive-stranded RNA viruses at $26-32 \mathrm{~kb}$, and are divided into four genera: $\alpha-, \beta-, \delta-$, and $\gamma_{-}$CoVs. ${ }^{1,2}$ SARS-CoV- 2 has been identified and classified as lineage $B$ of the genus $\beta$-coronavirus, ${ }^{3}$ which also includes severe acute respiratory syndrome coronavirus (SARS-CoV) and Middle East respiratory syndrome coronavirus (MERS-CoV). SARS-CoV-2 shares 79.6 and $96 \%$ sequence identity with SARS-CoV and the bat coronavirus RaTG13, 4 respectively. Its genome contains fourteen open reading frames (ORFs), which can be divided into two parts. ORF1a and ORF1ab (Fig. 1a), located in the first two-thirds of the viral genome from the $5^{\prime}$-end, are directly translated into two polyproteins (pp1a and pp1ab) by cellular ribosomes. ${ }^{6}$ Subsequently, the two polyproteins are processed by two viral proteases, papain-like protease (PLpro) and main-protease (Mpro), to produce sixteen nonstructural proteins (Nsps), Nsp1-Nsp16. ${ }^{7}$ Collectively, these constitute the replicationtranslation complex (RTC). ${ }^{8}$ RNA-dependent RNA Polymerase $(\mathrm{RdRp})$ is required for the expression of the remaining one-third of the genome. Notably, replication of the viral genome is also mediated by $\operatorname{RdRp}^{9}$ Subgenomic RNAs utilize the transcription and translation systems of the host to synthesize four structural proteins: spike (S), membrane (M), envelope (E), and nucleocapsid
(N), as well as several accessory proteins (ORF3a, ORF3b, ORF6, ORF7a, ORF7b, ORF8, ORF9b, ORF9c, and ORF10). ${ }^{10-12}$ Finally, RNA and structural proteins are assembled into the mature viral progeny, which are released by exocytosis to further infect the host (Fig. 1b).

The severity of the ongoing COVID-19 pandemic has raised an urgent need to develop antiviral drugs, vaccines, and antibodies. Prophylactic vaccines, which stimulate the host to produce humoral and cell-mediated immune responses, are the primary measure currently used for the prevention of SARS-CoV-2 infection. The type of vaccines available includes the following: (1) inactive or live attenuated whole virus vaccine (US20060039926 ${ }^{13}$ and CoronaVac [Sinovac Biotech in China]); (2) nucleic acid vaccines, including DNA and mRNA vaccines, such as ino-4800 and mRNA-1273; ${ }^{14}$ (3) recombinant protein vaccines, including recombinant $S$ protein vaccines, recombinant $S$ protein subunit vaccines, ${ }^{15}$ and virus-like particle vaccines; (4) viral vector vaccines, including replication-incompetent vector vaccines, replication-competent vector vaccines, and inactivated virus vectors such as adenoviral vector vaccine; ${ }^{16}$ and (5) other types of vaccines, such as Bacille Calmette-Guerin (BCG) Vaccines. ${ }^{17}$ Moreover, various potential drugs have been proposed for the treatment of COVID-19. These can be divided into the following groups: (1) chemical medicines, such as nucleoside analogs (chloroquine, hydroxychloroquine, remdesivir, tenofovir, and sofosbuvir) $i^{18,19}$ (2) Traditional Chinese medicines, such as Lianhua Qingwen; ${ }^{20}$ and (3) biological agents, including antibodies, vaccines, peptides, oligonucleotides (aptamer, antisense

\footnotetext{
'Division of Respiratory and Critical Care Medicine, Respiratory Infection and Intervention Laboratory of Frontiers Science Center for Disease-Related Molecular Network, State

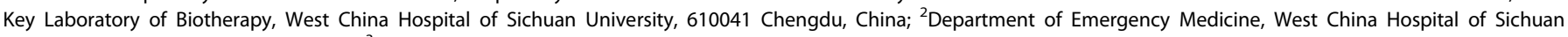
University, 610041 Chengdu, China and ${ }^{3}$ The First People's Hospital of Longquanyi District Chengdu, 610100 Chengdu, China

Correspondence: Bin He (hb40400387@163.com) or Wei Cheng (chengweilab@163.com)

These authors contributed equally: Weizhu Yan, Yanhui Zheng, Xiaotao Zeng
}

Received: 16 November 2021 Revised: 5 January 2022 Accepted: 10 January 2022

Published online: 27 January 2022 
a

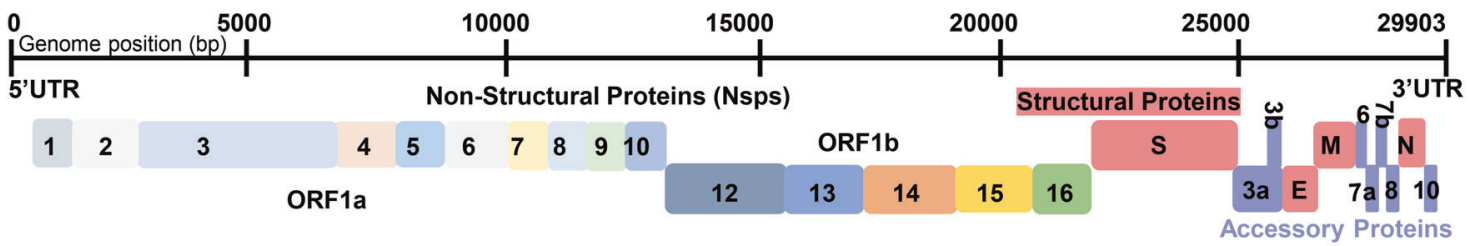

b

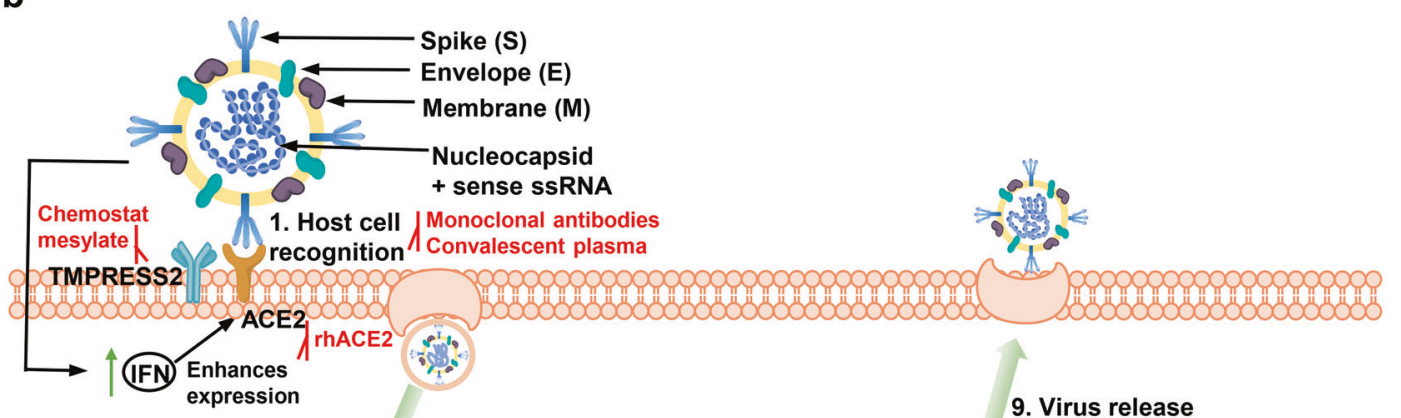

2. S2 subunit proteolysis, membrane fusion and viral

RNA release Arbidol Chloroquine

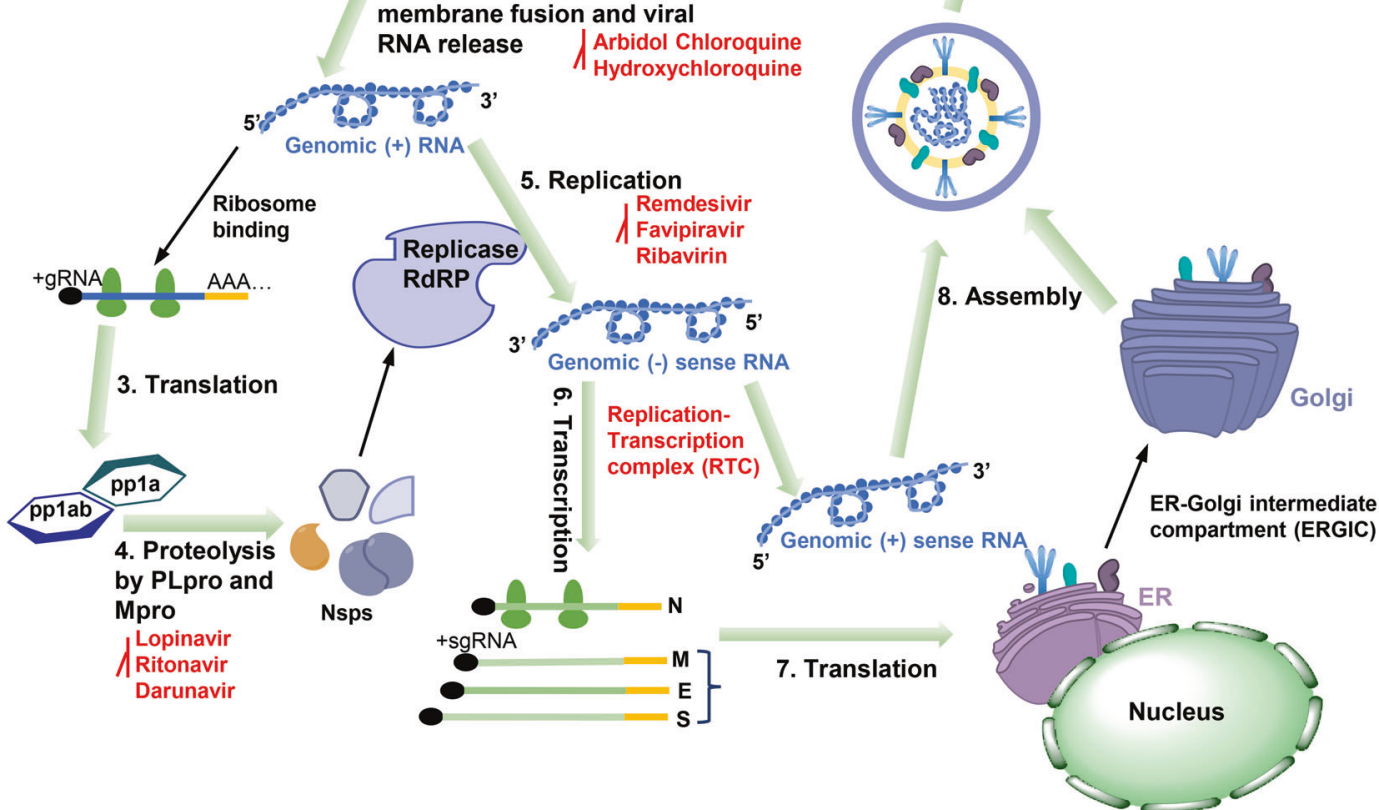

Fig. 1 The whole-genome composition and replication cycle of SARS-CoV-2 and potential targets. a The viral genome encodes 16 nonstructural proteins (Nsps) required for replication/transcription and structural proteins required for the assembly of new virions. $\mathbf{b}$ the SARS-CoV-2 mainly infects lymphatic epithelial cells and type II pneumocytes with the initiation of human body's innate response by producing interferons (IFNs). However, IFN activates expression of ACE2 protein which acts as receptor for virus attachment to host cells. Interaction between S protein and ACE2 leads to proteolytic cleavage at the S1-S2 boundary and S2' site mediated by transmembrane protease serine 2 (TMPRSS2), further inducing the viral and host cell plasma membrane fusion. The single-stranded RNA in the viral genome is translated by host machinery to produce viral polypeptides (pp1a and pp1ab), which undergo proteolytic cleavage by PLpro and Mpro proteins to synthesize Nsps. These Nsps encode replication transcription complex (RTC), which continuously replicates and produces a series of subgenomic messenger RNAs that encode the accessory and structural proteins. The viral genomic RNA and proteins are assembled to form the virus particles in the ER-Golgi intermediate compartment (ERGIC). The vesicle-containing virus then fuses with plasma membrane of the host, releasing the viral particles out of the cell The antiviral molecules with target sites are highlighted in red

oligonucleotides, small interfering RNAs [siRNAs], RNA interference [RNAi]), interferons, ${ }^{21}$ corticosteroids, ${ }^{22}$ plasma, ${ }^{23}$ and mesenchymal stem cells. ${ }^{24}$

Some efficient vaccines and drugs for emergency use have already been approved. However, the emergence of multiple mutants caused by various competitive processes originating from molecule aspect (such as reading frame shifts, replication errors, etc., of virus itself), organism aspect (such as gene re-edit and recombination induced by host adaptive immune response), and population aspect (such as natural selection) ${ }^{25}$ making it critical to identify alternative targets and develop more broad-spectrum antiviral drugs. ${ }^{26}$ Structural biology can be used to study the pathogenic mechanism of viruses, but also provides theoretical information for drug development and optimization. For this purpose, we summarize the representative structures of SARSCoV-2 and discuss the development of drugs, antibodies, vaccines, and other therapeutic agents for targeting these proteins or the virus. 


\section{STRUCTURAL PROTEINS OF THE VIRUS}

$S$ protein

The transmembrane $S$ protein, protruding from the viral surface, ${ }^{27}$ recognizes the host receptor angiotensin-converting enzyme 2 (ACE2), to mediate coronavirus entry into host cells. ${ }^{28} \mathrm{~S}$ protein remains the main target of many anti-coronavirus drugs, including neutralizing monoclonal antibodies (mAbs), vaccines, and other inhibitors. Further study of its structure will contribute to the development of more effective or broad-spectrum antiviral drugs.

SARS-CoV-2 $\mathrm{S}$ protein is cleaved by host proteases into two subunits, a receptor-binding fragment (S1) and a fusion fragment (S2), during biogenesis or virus assembly. The S1 subunit consists of an N-terminal domain (NTD, residues 14-306), a receptorbinding domain (RBD, residues 331-528) including receptorbinding motif (RBM, residues 436-506), and the C-terminal domains (CTDs) composed of CTD1 (residues 528-591) and CTD2 (residues 592-686). The S2 subunit is divided into the fusion peptide (FP, residues 816-836), fusion-peptide proximal region (FPPR, residues 837-857), heptad repeat 1 (HR1, residues 911-985), central helix ( $\mathrm{CH}$, residues 986-1036), connector domain (CD, residues 1037-1068), heptad repeat 2 (HR2, residues 1164-1211), transmembrane segment (TM, residues 1212-1234), and cytoplasmic tail (CT, residues $1235-1273)^{29}$ (Fig. 2a).

The S1 subunit of the $S$ trimer wraps around the threefold axis, covering the S2 subunit underneath. The NTDs are located at the periphery of the $S$ trimer and make contact with the RBD from the adjacent protomer. ${ }^{29}$ The RBD is located at the apex of the S1 subunit, which is a key region for coronavirus invasion of host cells. Before and after S protein binds to ACE2, the RBD undergoes a hinge-like conformational change that transiently switches between open (or up) and closed (or down) states. ${ }^{30}$ The open state is required for ACE2 engagement, because the RBM is embedded inside the protein during the closed state. ${ }^{28,30}$ In the S-ACE2 complex, four pairs of disulfide bonds (C336-C361, C379-C432, C391-C525, and C480-C488) are involved in stabilizing the RBD structure, and the RBM forms a concave outer surface to accommodate the $\mathrm{N}$-terminal helix of ACE2. There are 13 hydrogen bonds, two salt bridges (between K417 of the RBD and the D30 of ACE2), and several hydrophobic interactions (between F486 of the RBD and L79, M82, and Y83 of ACE2) contributing to ACE2 engagement ${ }^{28,30}$ (Fig. 2b, c). Notably, the salt bridge interactions between $\mathrm{K} 417$ (outside the RBM region) of SARSCoV-2 S protein and D30 of ACE2 is absent in SARS-CoV. ${ }^{30}$ The CTDs, which is mainly consisted of $\beta$-structure, also play an essential role in the structural rearrangement of $S$ protein membrane fusion. ${ }^{29}$ The CTD1 and CTD2 wrap around S2 and are adjacent to RBD and NTD, respectively. And the CTD1 is located between the two NTDs and interacts with NTD from another protomer. A special structural element "630 loop", located near the S1/S2 boundary and structurally inserted the gap between the NTD and CTD1 of the same protomer, appears in the CTD2 and stabilizes the closed conformation of S protein. ${ }^{29}$

The cleavage of the S protein into S1 and S2 occurs before the fusion of the virus and the host cell membrane, which is necessary for the viral entry into the host cell. And the S2 is further cleaved by TMPRSS2 to FP and S2' and undergo significant conformational changes during the membrane fusion ${ }^{31,32}$ (Fig. 2b). In the prefusion state, the S2 subunit adopts a conformation packed around a three-strand coiled-coil formed by central $\mathrm{CH}$. A nine helix-bundle is assembled by the central coiled-coil and helix structure of the HR1 together with another helix structure formed by residues $758-784,{ }^{33}$ which likely stabilizing the overall structure of $\mathrm{S}$ trimer. The FPPR and FP are connected directly via a longer loop. And the FPPR tucks underneath CTD1 from another protomer and interacts with CTD2 and HR1 of the same protomer. However, the HR2, TM, and CT regions are disordered in most of the $\mathrm{S}$ protein structures that have been determined, except for the low-resolution density that can be observed in cryo-ET reconstruction. In the postfusion state, HR1 undergoes a significant refolding transition, and a long central three-strand coiled-coil of $\sim 180 \AA$ As assembled by a continuous a-helix formed by HR1 and $\mathrm{CH}^{29}$ Besides, HR2 partly becomes ordered and packs against the groove of HR1 coiled-coil with the formation of sixhelix-bundle $(6 \mathrm{HB})$ structure, which contribute to the fusion of virus and cell membrane. ${ }^{34,35}$

Antibodies, vaccines, and inhibitors targeting the $S$ protein A range of antiviral agents targeting the $S$ protein has been developed, such as small molecular inhibitors, antibodies, and vaccines. Generally, inhibitors targeting $S$ glycoproteins block virus-membrane fusion by competitively inhibiting the interaction between RBD and ACE2. Such inhibitors include ivermectin ${ }^{36,37}$ and arbidol (umifenovir). ${ }^{38-40}$ Some also target non-RBD regions, such as lipopeptide EK1 (and its derivative, EK1C4), ${ }^{41}$ a pancoronavirus fusion inhibitor that targets HR1 of the S2 domain and inhibits membrane fusion.

Monoclonal antibodies elicited from memory B cells of vaccinated or SARS-CoV-2-infected individuals are also effective in prophylaxis or treatment of COVID-19. A number of potential antibodies against SARS-CoV-2 have been identified, some of which target the NTD or S2 domain, and a high proportion of antibodies target RBD. According to the distinct epitope landscape of RBD-directed antibodies, these mAbs can be classified into seven communities (RBD-1 to RBD-7), and each community can be further divided into finer clusters and bins. ${ }^{42}$ Nine antibody types including NTD-, RBD-, and S2-directed antibodies and the mechanism of each are described below.

NTD mAbs (class I, such as 4A8, ${ }^{43} \mathrm{FCO}^{4},{ }^{44} \mathrm{CM} 25,{ }^{45}$ and $\mathrm{S} 2 \mathrm{M} 28^{46}$ ) primarily targeted a "supersite"(consisted by residues 14-20, $140-158$, and $245-264$ ) of the NTD ${ }^{46}$ (Fig. 2d). Their neutralizing activity does not rely on the sterically inhibition of ACE2 binding, 46 whereas likely block S-mediated virus-cell fusion by preventing interaction with its auxiliary receptor, proteolytic activation, and membrane fusion. ${ }^{46}$ Antibodies in this group are conformationally sensitive and also affected by mutations other than epitopes, ${ }^{42}$ such as several frequent NTD-located deletions $(\Delta 69-70, \Delta 144$, $\Delta 157-158$, and $\Delta 211$ and $\Delta 242-244$ ) in circulating VOCs (alpha and Omicron variant) (Fig. 2f).

Three RBM-directed antibodies (RBD-1/RBD-2/RBD-3 mAbs) recognize $\mathrm{RBD}$ in a manner nearly identical to that of ACE2 (Fig. 2g), meaning that the opened RBD conformation is required in the antibodies binding process. The differentiation lies in their respective epitope landscape, where RBD-1 mAbs (class II; such as CoVIC-259, EMD-24335) largely overlap with the RBM; RBD-2 mAbs (class III; such as CoVIC-252, CoVIC-010, CoVIC-140, and CoVIC-002) shift from the center of the ACE2 binding site toward the "Peak" of the RBM (consisted by residues F486, S477, T478, and E484); and RBD-3 mAbs (class IV; such as CoVIC-080, and EMD-24346) bind down from the center of ACE2 binding site toward the RBM "Mesa" (consisted by residues N501). These three types of antibodies block RBD-ACE2 interactions by both steric hindrance and direct competition for interface residues. Two classes of RBD mAbs (RBD-4 and RBD-5) can bind to the outer face of the open or closed RBD without steric hindrance (Fig. 2h), in which RBD-4 mAbs (class V; such as CoVIC-094, and EMD-24350) bind toward the outer edge of the RBM and most of them can block ACE2, whereas the epitope of RBD-5 nAbs (class Vl; such as CoVIC-134, and EMD-24384) are away from the RBM and have weakly blocking to ACE2 binding. ${ }^{42}$ The remaining two groups of RBD mAbs (RBD-6 and RBD-7) recognize the inner face of the RBD, and two open RBDs are required in its targeting process (Fig. 2i). The main difference between RBD-6 (class VIl; such as CoVIC-250, and CoVIC-028) and RBD-7 (class VIII; such as CoVIC-063, CR3022, ${ }^{47}$ and CoVIC-021) is their competition variance with RBD-2 (especially RBD-2a) antibodies, and the latter is superior to the former. ${ }^{42}$ All RBD-6 and partial RBD-7 (RBD-7a) antibodies are ACE2 blocking, 
a

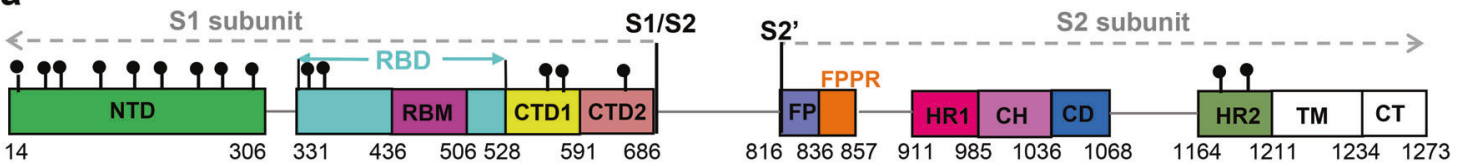

b

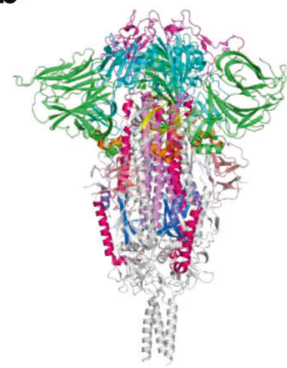

S-prefusion (PDB ID: 6XR8)

d

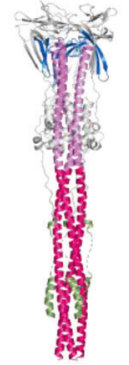

S-postfusion (PDB ID: 6XRA)
C

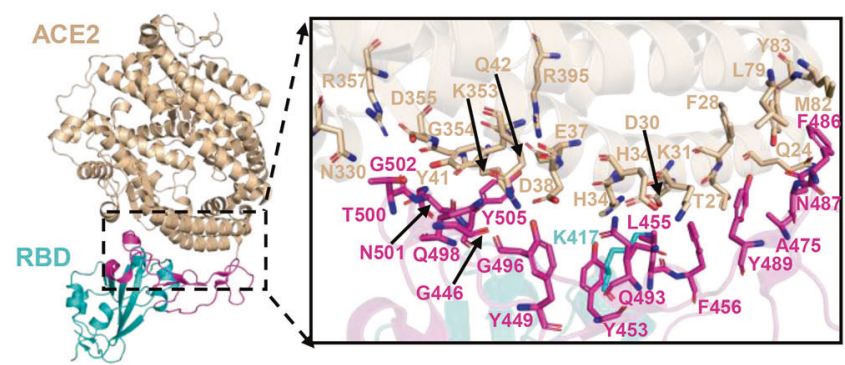

S-RBD + ACE2

(PDB ID: 6MOJ)

e

$\mathbf{f}$

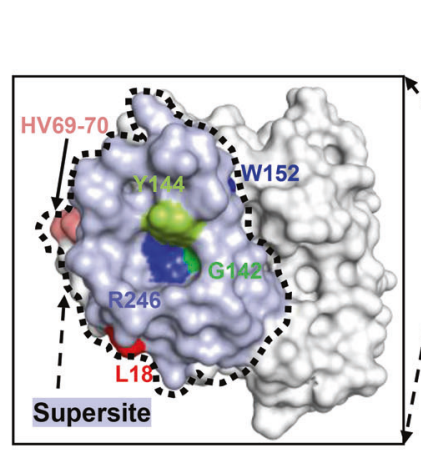

g

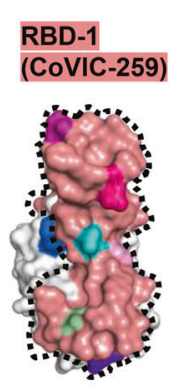

RB-Top

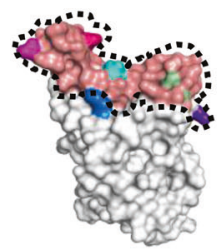

RBD-Inner

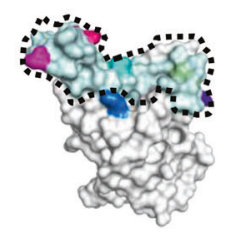

RBD-Inner

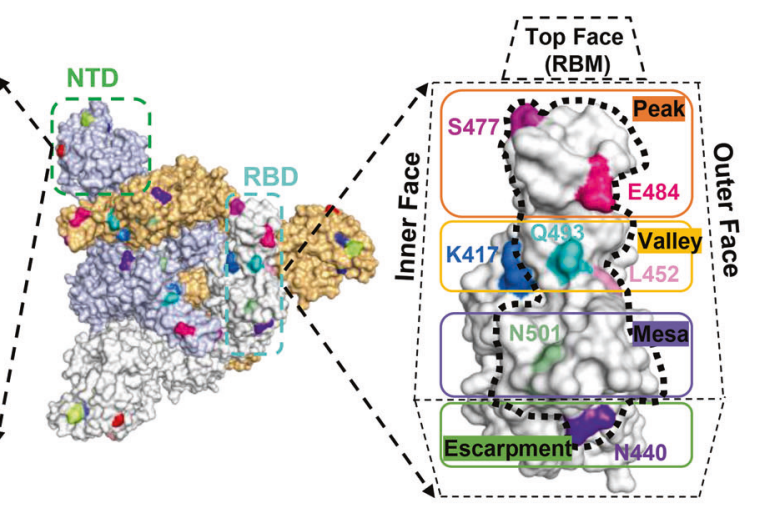

h

RBD-3
(CoVIC-080)

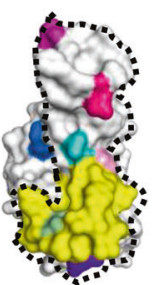

RBD-Top

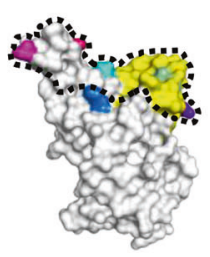

RBD-Inner

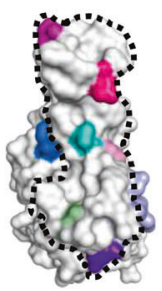

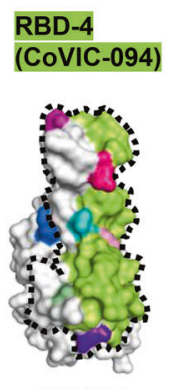

RBD-Top

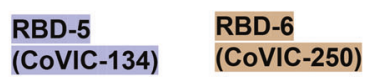

RBD-Top

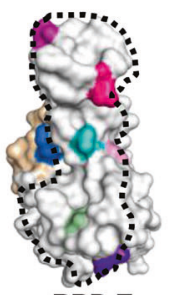

RBD-Top
RBD-7 (CoVIC-063)

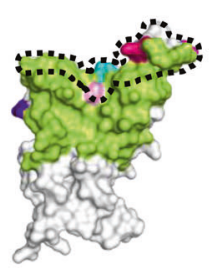

RBD-Outer

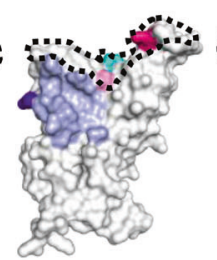

RBD-Outer

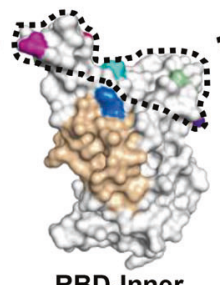

RBD-Inner

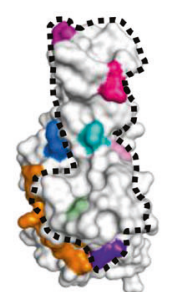

RBD-Top and the rest of RBD-7 (including RBD-7b [such as CR3022] and RBD-7c [such as CoVIC-21]) are not ACE2 blocking. ${ }^{42}$

Only a few S2 mAbs (class IX) including have been reported to date, such as CC40.8, ${ }^{48}$ and $1 \mathrm{~A} 9,{ }^{49}$ both of which belong to the cross-reactive antibody. Unlike 1A9, CC40.8 can interact with
S2 subunit and neutralize SARS-CoV-2, while 1 A9 only interacts with S2 subunit. In addition to the monoclonal antibodies mentioned above, polyclonal antibodies (pAbs) and antibody cocktails have also been proposed to combat the constantly emerging mutant strains. ${ }^{28,50}$ 
Fig. 2 Structures of SARS-CoV-2 Spike protein and antibody recognition. a The full-length SARS-CoV-2 S protein. NTD N terminal domain, RBD receptor-binding domain, RBM receptor-binding motif, CTD1 C-terminal domain 1, CTD2 C-terminal domain 2, FP fusion peptide, FPPR fusionpeptide proximal region, HR1 heptad repeat 1, CH central helix region, CD connector domain, HR2 heptad repeat 2, TM transmembrane segment, CT cytoplasmic tail. b Structures of S trimer in prefusion and postfusion states. Each domain is marked with a color corresponding to a. c Structure of the RBD (cyan) in complex with ACE2 (pink). Residues involved in interactions between the RBD and ACE2 are shown as sticks. d Footprints for NTD-targeted antibodies, with the NTD "supersite" outlined with a dashed line. The residue positions of important mutations and deletions are indicated in the NTD. Table 1 lists mutations represented in each variant. e Location of important emerging mutations on the RBD. The RBM can be topologically divided into three subsections: the "peak" that includes residues S477 and E484; the "valley" that includes K417, Q493, and L452; and the "mesa" includes N501. f Mutations and deleted residues affecting antibodies activity involved in significant mutants. g-i footprint of a representative antibody from the Coronavirus Immunotherapeutic Consortium (CoVIC) mapped onto an RBD monomer. The ACE2 binding site is outlined with a dotted line. The website of CoVIC is at https://covic.lji.org/

There are currently 137 COVID-19 vaccines in clinical development, of which recombinant protein subunit vaccines account for 35\% (https://www.who.int/publications/m/item/draft-landscapeof-covid-19-candidate-vaccines). In the process of vaccine development, a series of obstacles have been encountered. For example, full-length recombinant $S$ glycoprotein is difficult to express and has poor stability; recombinant RBD vaccines recognize fewer neutralizing epitopes compared with full-length $S$ protein vaccines and are prone to antigenic drift; ${ }^{16}$ and the extensive glycosylation of $\mathrm{S}$ glycoprotein makes it easy for the coronavirus to escape host immune attack. ${ }^{51,52}$ Employing the methods of structural determination, some groups have found that the more stable closed prefusion $S$ glycoprotein can be obtained by introducing single point mutations and disulfide bridges. ${ }^{53,54}$ Furthermore, several key residues associated with glycosylation have been identified, including N165, N234, and T323 of the S protein. ${ }^{55-57}$ All of this structural information provides an important theoretical basis for further vaccine optimization.

In addition to the $S$ protein, other proteins involved in the process of virus-membrane fusion can also be targeted. ACE2 is a host receptor of $\mathrm{S}$ protein and is highly expressed in many tissues (including nasal mucosal, bronchus, lung, heart, kidney, and intestinal tissue $^{58}$ and regulated by the amino-acid transporter $B^{0}$ AT1. ${ }^{59}$ Some inhibitors (such as DX600, ${ }^{60}$ MLN4760, ${ }^{61}$ and $\mathrm{NAAE}^{62}$ ) targeting ACE2 effectively inhibit entry of the virus. Host proteases, such as cathepsins, ${ }^{63}$ furin, ${ }^{28}$ TMPRSS2 $^{64}$ and trypsin are another set of potential targets. The $S$ protein is cleaved by host proteases at the $\mathrm{S} 1 / \mathrm{S} 2$ and S2' sites, activating the S protein and allowing it to mediate membrane fusion. ${ }^{65,66}$ Inhibitors of furin protease include decanoyl-RVKR-chloromethylketone $(C M K)^{67}$ and a-1 antitrypsin Portland (a1-PDX). ${ }^{68}$ There are other known host receptors of $S$ protein, such as glucose-regulated protein 78 (GRP78) ${ }^{69}$ and CD147, ${ }^{70}$ which could also be targeted.

Effect of S protein mutations on SARS-CoV-2 immune evasion To date, there are five globally recognized SARS-CoV-2 variants of concern (VOCs; Alpha, Beta, Gamma, Delta, and Omicron) and several variants of interest (VOls; Epsilon, Zeta, Eta, Lota, Theta, Kappa, Lambda, and $\mathrm{Mu}$ ) are emerged under the pressure of natural selection of the human immune system. Among these variants, the most diverse regions in virus genomic are distributed in NTD and RBD of S protein (Table 1). These mutations have direct implications on virus virulence (infectivity or reinfection, transmissibility, disease severity, neutralization resistance to antibodies elicited by infection or vaccination), and thus potentially resulted in more obstacles to the precaution, diagnose, and therapy of COVID-19. The common characteristics of these variants and factors associated with virus virulence variance are discussed below.

Alpha variant has increased transmissibility, and disease severity than pre-existing variants. This variant has seven missense mutations and three deleted residues in the $S$ protein (Table 1). Among these mutations, N501Y mutation is shared by several VOCs (Alpha, Beta, Gamma, and Omicron) and VOls (Theta and
$\mathrm{Mu})$, and this mutation increase the binding affinity of S protein and ACE2 receptor by adding an additional hydrogen bond between them, and consequently raising the efficiency of viral infection and transmission. ${ }^{71}$ D614G mutation increases the ability of RBD to shift to the up position, and is more conducive to the transmission and infection of the virus. ${ }^{72} \mathrm{P} 681 \mathrm{H}$ mutation is adjacent to the furin cleavage site and could potentially have an effect on S1/S2 cleavage and therefore on cell entry and infectivity. ${ }^{73}$ Three deleted residues in NTD $(69-70$, Y144) are recurrent mutation sites observed in VOCs and VOls, and likely responsible for the resistance of these variants to neutralization by NTD-specific antibodies. ${ }^{74}$ Fortunately, the Alpha variant remains sensitive to neutralization by currently potential antibodies, but its level is moderately reduced. ${ }^{75}$

The second dominant VOCs is Beta variant, and $\sim 77 \%$ of mutations in its genomic located in the $S$ protein include seven missense mutations and three deleted residues (Table 1). ${ }^{75}$ Multiple studies have shown that Beta variant displayed neutralization resistance to most NTD-, and RBM-specific mAbs, and this resistance is mainly ascribed to three mutations within RBD (K417N, E484K, and N501Y). ${ }^{76}$ N501Y probably does not impair neutralization on its own but rather in combination with $\mathrm{K} 417 \mathrm{~N}$ and $\mathrm{E} 484 \mathrm{~K}$, both mutations play contributions to the enhanced binding affinity of S protein with ACE2 and increased immune evasion capability of variants. ${ }^{75}$ Furthermore, the combination of RBD and NTD mutations in the S protein would affect the neutralization more significantly.

The Gamma variant is the third VOC. This variant contains eleven S protein mutations, including five mutations within NTD (Table 1), three mutations in RBD that resemble to RBD mutations in the Beta variant (K417N, E484K, and N501Y), two mutations near the furin cleavage site (D614G and H655Y), and one mutation in S2 (T1027I). Gamma variant has significant resistance to the neutralizing antibodies from convalescent and vaccinee sera, but the degree of resistance is not as severe as against Beta and only slightly weakened compared to that of Alpha. ${ }^{77}$ The reason for the differences in neutralization resistance of Beta and Gamma presumably reflects the difference in the mutations accumulated outside the RBD. ${ }^{77}$

The presently dominated VOCs in the global is still Delta variant, which may be over $60 \%$ more transmissible than the Alpha variant. $^{77}$ This variant is characterized by numbers of spike mutations, including five NTD mutations (Table 1), three RBD mutations (L452R, T478K, and E484K), and two mutations near the furin cleavage site (D614G and P681R), and one S2 mutation (D950N). L452R mutation has been proved to be associated with increased infectivity, higher transmission, and immune evasion of SARS-CoV-2 variants. ${ }^{78}$ T478K mutation was previously selected in vitro and shown to exhibit reduced neutralization by monoclonal antibodies and human convalescent sera. ${ }^{79}$ The significantly increased immune escape capacity to neutralize antibodies of this variant may be related to the RBD mutations and their combination with NTD mutations.

The recently emerged Omicron variant, with higher transmissibility, infection/reinfection rate, and enhanced ability of immune 


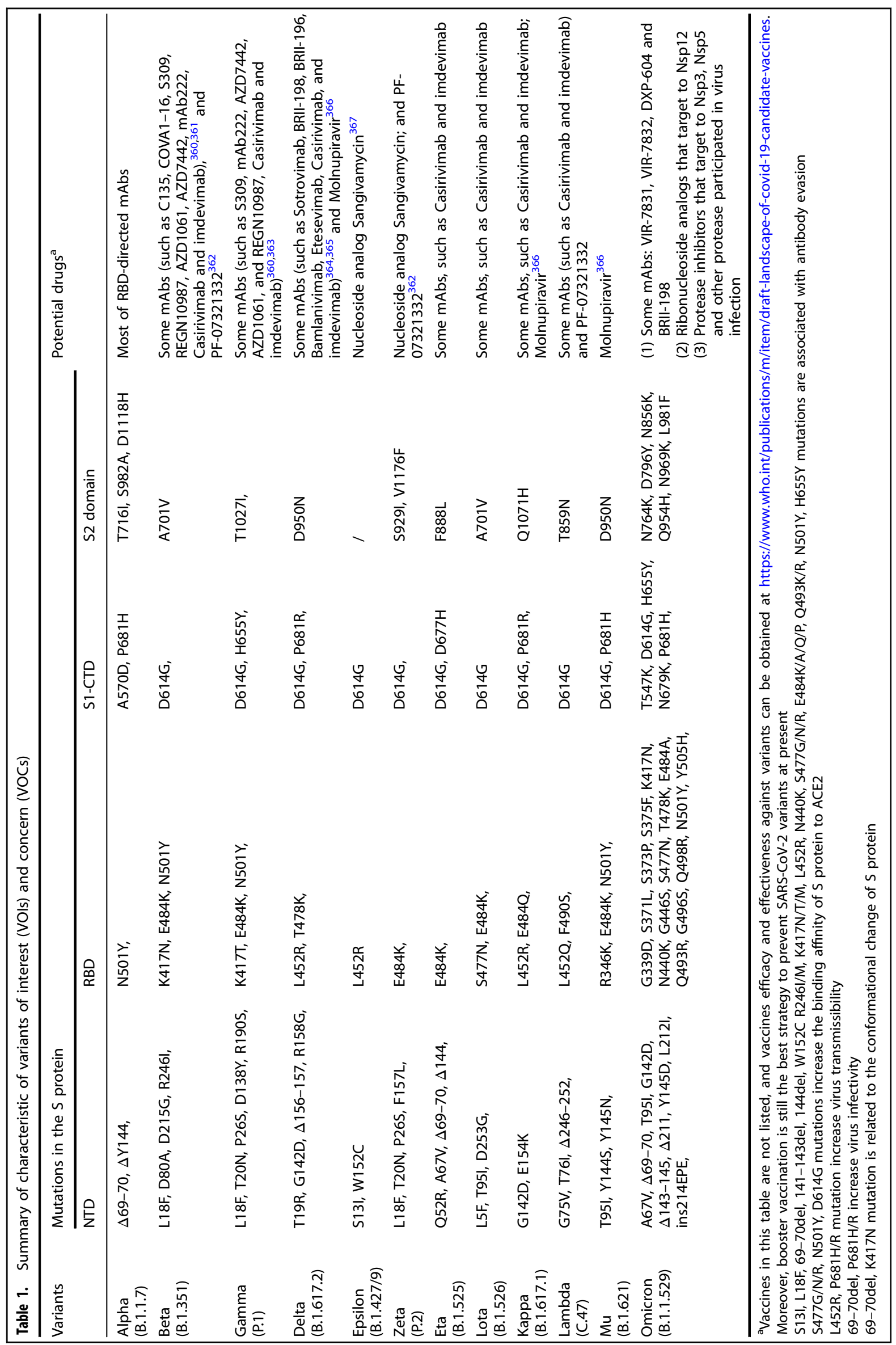


Table 2. Overview of targets and potential drugs of SARS-CoV-2

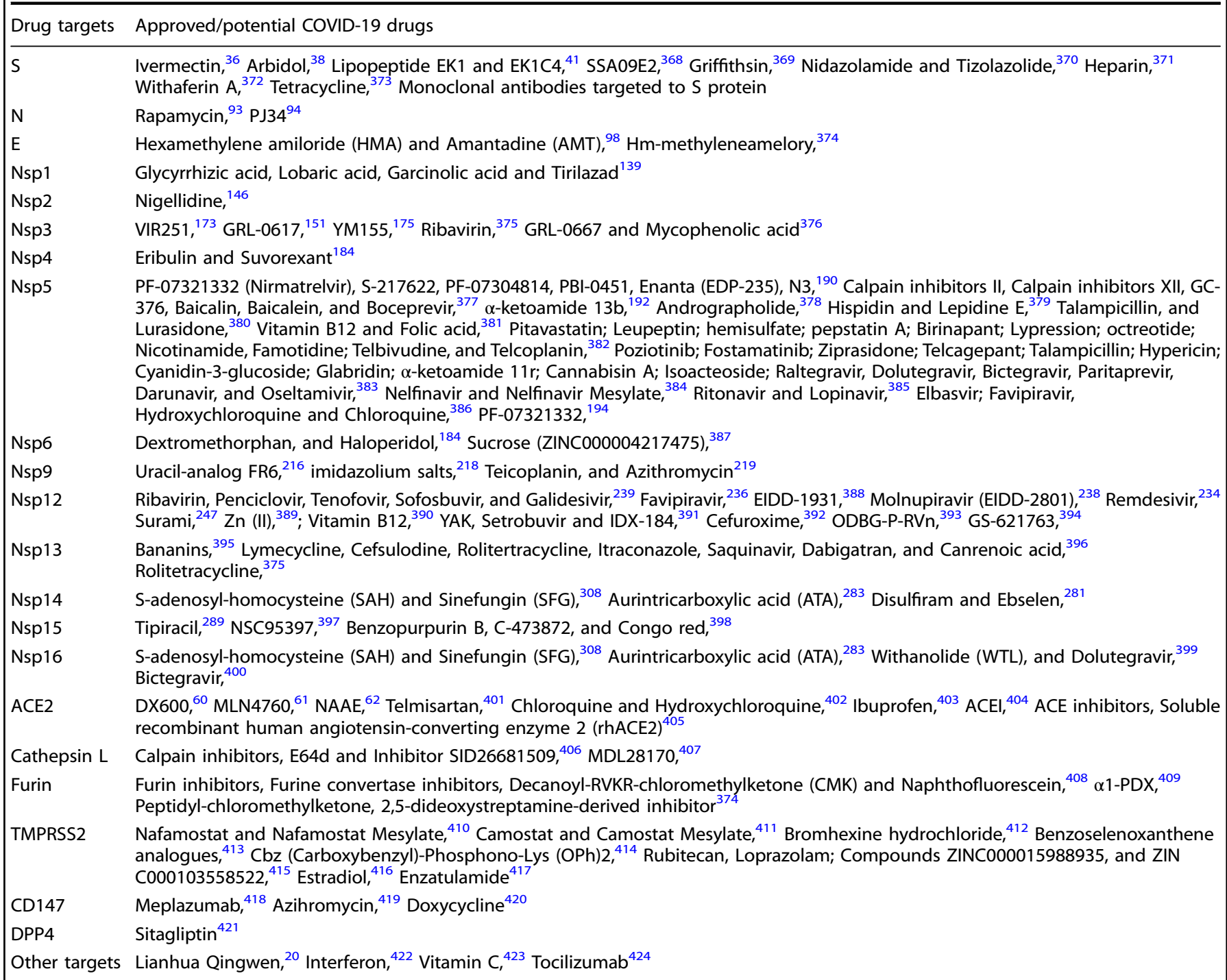

escape, are attracting more and more attention. ${ }^{80}$ This variant has more than 50 mutations, and most of the mutations (at least 32) were located within S protein, with half of these mutations are located in the RBD (Table 1) and 10 of them are concentrated in the RBM. ${ }^{81,82}$ Although some mutations (such as $\triangle 69-70$, T95I, G142D, $\Delta 143-145, \mathrm{~K} 417 \mathrm{~N}, \mathrm{~T} 478 \mathrm{~K}, \mathrm{~N} 501 \mathrm{Y}, \mathrm{H} 655 \mathrm{Y}, \mathrm{N} 679 \mathrm{~K}$, and $\mathrm{P} 681 \mathrm{H})$ that overlapped with those in other VoCs are have been investigated to participate in viral transmissibility, binding affinity, and immune escape; while the role of the most of the remaining mutations is still not known. Therefore, it is urgent to study the effect of these mutations on virus virulence, antigenicity, and epidemiology, as well as neutralization activity to antibodies. In addition to the mutations in S protein, only one mutation in each of these genes (Nsp14 and Nsp5) are found, so inhibitors that are targeted these proteins may be useful for Omicron variant. ${ }^{83}$ Some approved or potential representative drugs targeting SARS-CoV-2 proteins were listed in Table 2.

Some essential mutations mentioned above (L452R, E484K/Q, N501Y, D614G, and P681H/R) also presented with some significant VOI variants, such as Epsilon, Eta, Lota, Kappa, Zeta, and Theta. Their respective mutation information in $S$ protein is listed in Table 1.
$\mathrm{N}$ protein

The multifunctional nucleocapsid $(\mathrm{N})$ protein not only participates in viral replication and assembly, but also interferes with the interferon pathway of the host. ${ }^{84,85}$ Compared with the more researched drug targets (S protein, 3CLpro, and PLpro), the $\mathrm{N}$ protein has several advantages: it has higher sequence conservation, is less prone to mutation, and induces a stronger protective immune response in the host. Therefore, $\mathrm{N}$ protein is a great potential target for diagnosis.

The SARS-CoV-2 N protein shares $90 \%$ homology with the SARSCoV N protein. ${ }^{86}$ SARS-CoV-2 $\mathrm{N}$ comprises 419 amino acids, which are divided into the $\mathrm{N}$-terminal domain (residues 49-174), the C-terminal domain (CTD, residues 247-364), and a flexible linker (residues 175-246) (Fig. 3a). The linker is rich in serine/arginine (SR, residues 176-206) and leucine/glutamine (LQ, residues 210-246), and SR regions play an essential role in primary phosphorylation. ${ }^{87}$ The structure of the full-length $\mathrm{N}$ protein has not yet been resolved, but specific structural features are known. The N-NTD folds into a right-hand shape, in which six antiparallel $\beta$-sheets $(\beta 4-\beta 2-\beta 3-\beta 1-\beta 5-\beta 6)$ form the palm and a protruding positively charged $\beta$-hairpin located between $\beta 2$ and $\beta 3$ forms the basic finger ${ }^{88}$ (Fig. 3b). A typical characteristic of the $N$ protein is that it 
a

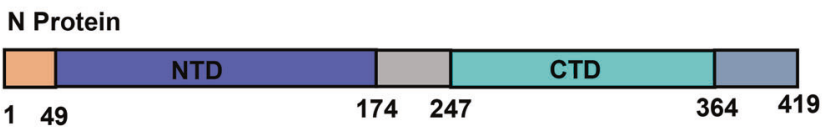

b

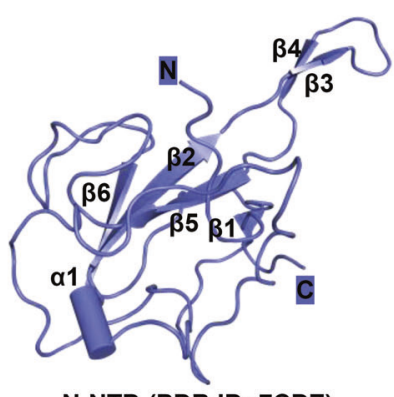

N-NTD (PDB ID: 7CDZ)
C

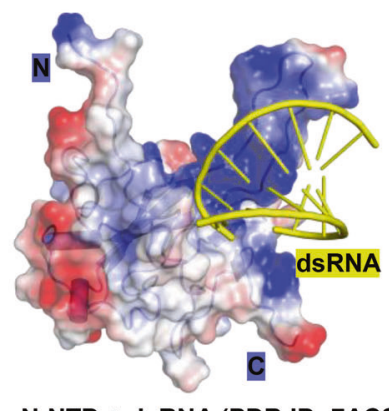

N-NTD + dsRNA (PDB ID: 7ACS)

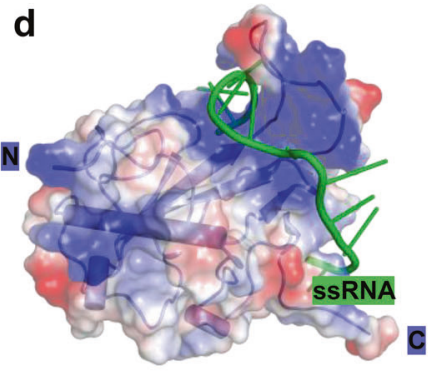

N-NTD + ssRNA (PDB ID: 7ACT) e

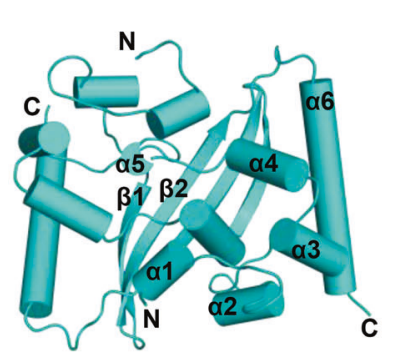

N-CTD (PDB ID: 6WZO)

g
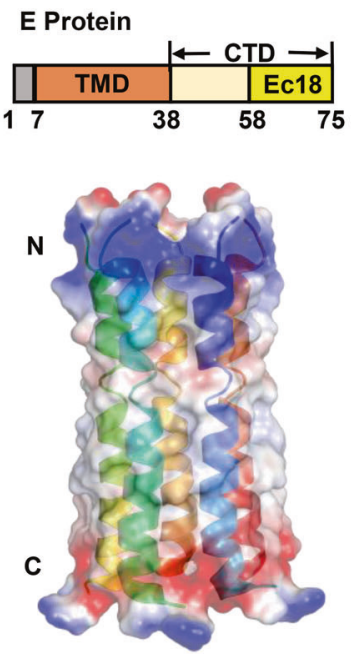

E-TMD

(PDB ID: 7K3G) f

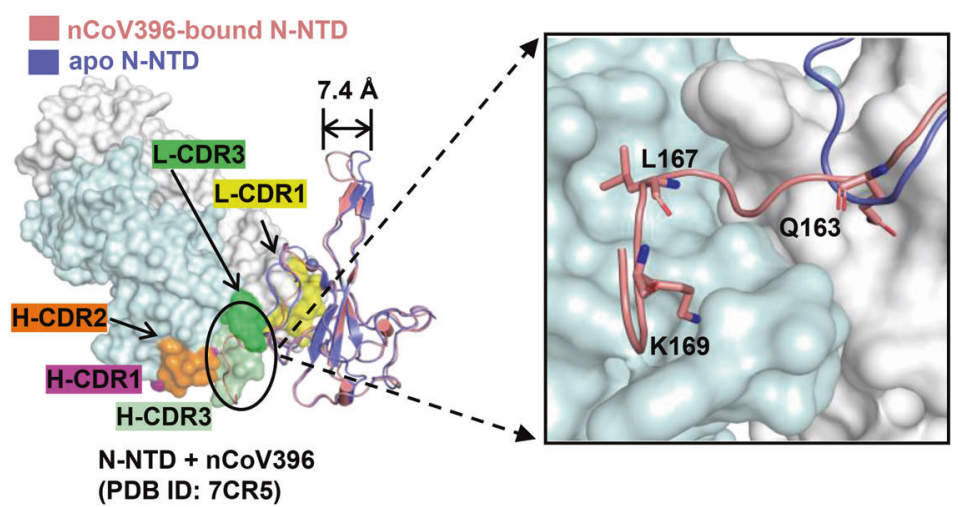

i h

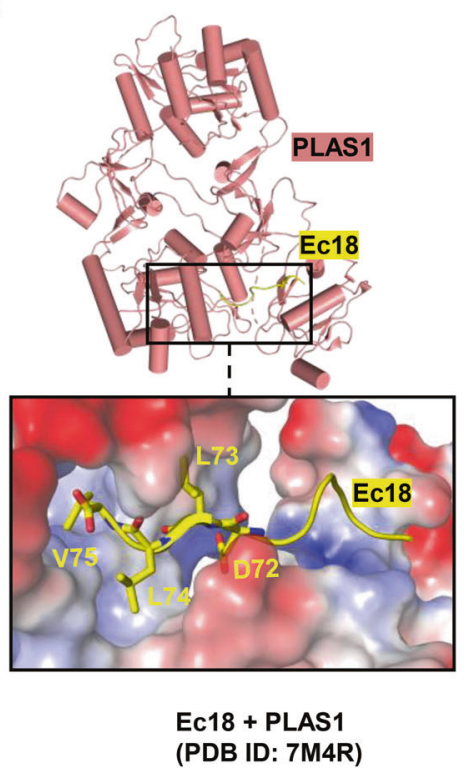

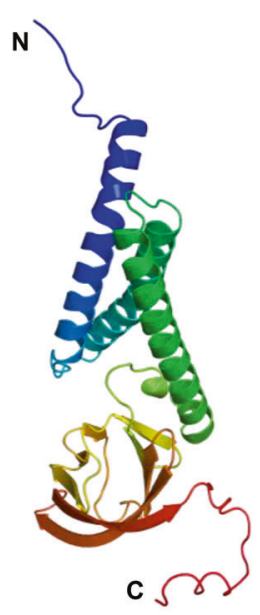

M protein (AlphaFold2)

Fig. 3 Structures of SARS-CoV-2 Nucleocapsid, Envelope, and Membrane proteins. a Schematic representation of N protein organization. NTD $\mathrm{N}$-terminal domain, CTD C-terminal domain. b Overall structures of N-NTD. c, d Overall structures of N-NTD in complex with ssRNA and dsRNA, respectively. The N-NTD is illustrated with electrostatic surface. e Overall structure of N-CTD. $f$ Conformational changes between apo and nCoV396-bound N-NTD. The monoclonal antibody nCoV396 is illustrated with surface. The key residues of interaction between nCoV396 and N-NTD are shown on the right as sticks. g Overall structure of SARS-CoV-2 E protein shown in surface and cartoon. TMD transmembrane domain, CTD C-terminal domain, Ec18 residues 58-75 of E-CTD. $\mathbf{h}$ Structure of the C-terminus of E protein (Ec18) in complex with human cell junction protein PLAS1. The Ec18 is shown in yellow. i Predicted structure of M protein using alphafold 2 
binds to viral RNA and forms a ribonucleoprotein complex (RNP). ${ }^{89}$ In the N-RNA oligonucleotide complex (Fig. 3c, d), the negatively charged RNA binds to the positively charged canyon in the $\mathrm{N}$ protein. RNA interacts with several basic residues (R92, R107, and R149) and causes a significant conformational change in the basic finger. ${ }^{85,90}$ The CTD domain of the $\mathrm{N}$ protein is assembled into a cuboid composed of homologous dimers, and each protomer consists of six $a$-helices and two $\beta$-strands (Fig. 3e). The a-helices and $\beta$-strands of one protomer are embedded in the cavity of the other protomer, forming four-stranded antiparallel $\beta$-sheets. Pairs of hydrogen bonds and hydrophobic interactions between the two adjacent $\beta$-sheets cooperatively stabilize the structure of the dimer.

The $\mathrm{N}$ protein is one of the most abundant structural proteins in virus-infected cells, ${ }^{91}$ and can be used as an immunodominant antigen to induce a protective immune response. Monoclonal antibody nCoV396 isolated from the blood of convalescent COVID-19 patients effectively interacts with the NTD of the N protein $(1.02 \mathrm{nM}) .^{92}$ Its heavy chain and light chain contain multiple CDRs. such as, L-CDR1 (residues 23-32), L-CDR2 (residues 51-54), L-CDR3 (residues 94 to 100), H-CDR1 (residues 26-33), $\mathrm{H}-\mathrm{CDR} 2$ (residues 51-57), and H-CDR3 (residues 99-108). These CDRs form hydrogen bonds and hydrophobic interactions with three residues of the N-NTD (Q163, L167, and K169) to stabilize the protein complexes. Notably, these three residues are relatively conserved in the highly pathogenic $\beta$-coronavirus $\mathrm{N}$ protein. nCoV396 causes a large allosteric regulation of the N-NTD, such as outward movement of the basic finger by $7.4 \AA$ and increased unfolding of the carbon end tail (residues 159-172) ${ }^{92}$ (Fig. 3f). These interactions synergistically benefit the antibody in neutralizing the antigenicity of the $\mathrm{N}$ protein. $\mathrm{nCoV} 396$ also has a high affinity with the $\mathrm{N}$ protein of SARS-CoV and MERS-CoV $(7.4 \mathrm{nM}){ }^{92}$ In the future, this antibody could be modified in a site-directed manner based on specific structural information to allow the production of more efficient and broader spectrum monoclonal antibodies. In addition to antibodies, some small molecules (such as rapamycin ${ }^{93}$ and $\mathrm{PJ} 34^{94}$ ) that interfere with the RNA binding of N-NTD and dimerization of N-CTD have also been recommended as inhibitors of the $\mathrm{N}$ protein.

\section{E protein}

The envelope (E) protein forms the cation-selective channel in the endoplasmic reticulum-Golgi intermediate compartment (ERGIC). ${ }^{95}$ The E protein not only assists in viral assembly, budding, and virulence ${ }^{96}$ but also induces the host NACHT, LRR and PYD Domain-Containing protein 3 (NLRP3) inflammasome. ${ }^{97}$ As a critical multifunctional structural protein, the $\mathrm{E}$ protein has attracted increasing attention from researchers.

SARS-CoV-2 encodes a transmembrane $E$ protein composed of 75 amino acids, including the $\mathrm{N}$-terminal ectodomain (residues 1 to 7), transmembrane domain (TMD) (residues 8-38), and C-terminal domain (residues 39-75), with the 18 amino acids at the C-terminus referred to as Ec18. The structure of TMD is a pentameric ion channel (PDB ID 7K3G) formed by five a-helices ${ }^{98}$ (Fig. $3 \mathrm{~g}$ ). The stability of the adjacent $\mathrm{a}$-helix and helical bundle is mediated by $\pi-\pi$ stacking (Phe23 and Phe26), Van der Waals packing (among the Val29-Leu31-lle33 triad), and extensive hydrophobic interactions (among the abundant hydrophobic residues in the pore of the channel). ${ }^{98}$ The guanidinium groups of two ion channel drugs, hexamethylene amiloride (HMA) and amantadine (AMT), interact with the polar amino acids at the entrance of the ion channel and occupy the amino-terminal lumen of the channel, blocking ion channel activity of the $\mathrm{E}$ protein. ${ }^{98,99}$ The postsynaptic density-95 (PSD-95), discs-large, zona occludens 1 (ZO-1) (PDZ)-binding motif (PBM: ${ }^{72}$ DLLV $^{75}$ ) at the C-terminus of the $E$ protein recognizes the PDZ domain of the human cell junction protein PALS1 and subsequently breaks the apical cell polarity complex formed by PALS1, Crumbs, and Pals1-associated tight junction protein (PATJ). ${ }^{100-102}$ This causes looseness and leakage of the lung epithelial junctions and facilitates viral spread and proliferation. ${ }^{103,104}$ In the PLAS1/Ec18 complex (Fig. 3h) (PDB ID 7M4R), two PALS1 proteins interact with one Ec18, and the DLLV motif of Ec18 occupies the hydrophobic pocket formed by the PDZ domain and SH3 domain of PLAS1. ${ }^{103}$ This pocket is also the binding site of the ERLI motif at the C-terminus of Crumbs (CRB-CT) in PLAS1 (PDB ID 4WSI). ${ }^{100,105}$ Therefore, similar peptide inhibitors derived from Ec18 can be designed to inhibit the interaction between the E protein and PLAS1. ${ }^{103}$ In addition to the two inhibitors mentioned above, several other drugs target the $\mathrm{E}$ protein, such as Bacillus Calmette-Guerin (BCG) vaccination; ${ }^{106}$ this is an alternative strategy for the treatment of COVID-19 that elicits specific host immunity targeting the SARS-CoV-2 E protein. ${ }^{107,108} \mathrm{~A}$ possible reason for this response is that a fragment of the $E$ protein (residues 17-29) has a high sequence identity with tuberculin-like proteins. ${ }^{107}$

Predicted structure of $M$ protein

Membrane glycoprotein (M), the most abundant protein in coronaviruses, $^{109}$ is the main component of the viral envelope and maintains the virion's size and shape. ${ }^{110}$ Moreover, M protein is involved in the processing, modification, and trafficking of multiple viral proteins, ${ }^{111}$ as well as the assembly and release of virus particles. ${ }^{112} \mathrm{M}$ protein also interferes with the host immune response through interferon antagonism. ${ }^{113}$ Therefore, it is a promising target for the treatment of COVID-19.

The SARS-CoV-2 M protein shares $90.5 \%$ sequence identity with SARS-CoV M protein. ${ }^{114}$ However, $M$ protein has been proven difficult to be expressed and purified, hindering progress in resolving the crystal structure and leaving only a predicted structure available. ${ }^{114}$ The SARS-CoV-2 M protein consists of 222 amino acids, including a short $\mathrm{N}$-terminus (residues 1-19), a triple-transmembrane domain (TM, residues 20-100), and a longer C-terminal cytoplasmic domain (residues 101-222). The predicted structure of the $\mathrm{M}$ protein is similar to the prokaryotic SemiSWEET sugar transport protein, ${ }^{115}$ with both containing three transmembrane helix bundles (Fig. 3i). The $M$ protein contains several conserved motifs, which are not only responsible for the homodimerization and translocation of the $M$ protein, but also participate in the interaction between the $M$ protein and other viral proteins. ${ }^{113,116}$ For example, "aromatic-XX-aromatic" regions (such as WLLW in TM2 [residues 50-70]) are closely related to the dimerization of the $\mathrm{M}$ protein, ${ }^{117}$ and the di-leucine motif located at the $C$-terminal tail of the $M$ protein interacts with the $\mathrm{N}$ protein. ${ }^{118}$ Research into these conserved motifs is expected to contribute to the development of drugs targeting this protein.

The SARS-CoV $M$ protein has strong immunogenicity and stimulates the host humoral response to produce neutralizing antibodies. ${ }^{119}$ Multiple cytotoxic T-lymphocyte $(C T L)$ related epitopes of $M$ protein have been identified by human leukocyte antigen (HLA) molecule (HLA-A ${ }^{*} 0201$ ), such as Mn2 (residues $88-96$ ) and Md3 (residues 60-69), located in the TM region. ${ }^{120}$ They all stimulate the host to produce a specific $\mathrm{CD}^{+} \mathrm{T}$ cell immune response. $^{120}$ In the HLA-A ${ }^{*} 0201-M n 2$ (PDB ID 316G) or HLA-A*0201-MD3 (PDB ID 316K) complex, several anchor residues (such as leucine, valine, methionine, and serine) in $\mathrm{Mn} 2 / \mathrm{Md} 3$ assist in tightly binding peptides to the HLA pocket. ${ }^{120}$ In the future, the same approach used to identify the CTL-related epitopes of the SARS-CoV $M$ protein can also be applied to screen the epitopes of the SARS-CoV-2 M protein, allowing for the design of peptide inhibitors or vaccines against this protein. Recently, some groups have used molecular dynamics (MD) simulations and other strategies to identify several potential drugs (such as remdesivir) with high affinity with the $M$ protein, ${ }^{121}$ but further studies are needed to confirm these interactions. 


\section{NONSTRUCTURAL PROTEINS OF SRAS-COV-2}

Currently, the drugs and vaccines used to treat or prevent COVID19 are predominantly targeted to the $S$ protein, but the rapid mutation in this region can easily lead to drug resistance. Therefore, it is necessary to develop drugs that target other proteins. SARS-CoV-2 encodes sixteen nonstructural proteins (Nsp1-Nsp16) that form the replication and transcription complex (RTC). The transmembrane proteins Nsp3, Nsp4, and Nsp6 hijack and rearrange the host endoplasmic reticulum membrane, then induce the formation of double-membrane vesicles (DMVs). ${ }^{122,123}$ As an organelle-like structure, the DMV is not only conducive to viral replication but also assists in the evasion of the host's innate immune response. Among these nonstructural proteins, the structure of the three transmembrane proteins is the most complicated, all consisting of multiple transmembrane domains and luminal domains. ${ }^{124}$ Nsp11 is a short peptide composed of 7 amino acids, ${ }^{125}$ and the structures of Nsp4 and Nsp6 remain unresolved; we, therefore, focus here on the other thirteen Nsps, and the predicted structures of Nsp4 and Nsp6 are also referred to.

\section{Nsp1 protein}

Nsp1 is an essential virulence factor of coronaviruses and is closely associated with the viral infection cycle and host translation regulation. ${ }^{126}$ It specifically binds to the host $40 \mathrm{~S}$ ribosomes and promotes endonucleolytic cleavage of host mRNA, thus hijacking the translation of multiple host genes. These genes include type-I interferon, ${ }^{127-129}$ allowing the virus to evade the host's innate immune defense. ${ }^{130,131}$ Therefore, recombinant viruses with mutated Nsp1 can be used to design live attenuated vaccines. $^{132,133}$

SARS-CoV-2 Nsp1 consists of 180 amino acids and shares high structural homology but low sequence identity with other Nsp1 proteins of $\beta$-coronaviruses (with the exception of SARS-CoV). In the $N$-terminal domain (NTD, residues 10-127) (PDB ID 7K7P), seven antiparallel $\beta$-strands assemble a closed $\beta$-barrel. An $\alpha$-helix is located at the opening of the barrel as a cap, and two $3_{10}$ helices are fixed on one side of the barrel. ${ }^{134}$ The C-terminal domain (CTD, residues 148-180) specifically interacts with the $40 \mathrm{~S}$ ribosomal subunit. ${ }^{135}$ In the Nsp1-CTD/40 S ribosome complex structure (Fig. 4a), Nsp1-CTD is embedded into the mRNA entry channel in the $40 \mathrm{~S}$ ribosome subunit. ${ }^{135-137}$ The stability of the complex depends on the electrostatic and hydrophobic interactions between Nsp1-CTD and $40 \mathrm{~S}$ ribosome protein subunits (uS3, uS5, and eS30), as well as the $18 \mathrm{~S}$ rRNA. The roles of Nsp1-NTD in the modulation of translation have been discussed; it not only stabilizes the binding of Nsp1-CTD to $40 \mathrm{~S}$ ribosome ${ }^{138}$ but also specifically interacts with stem-loop 1 (SL1) of the SARS-CoV-2 mRNA $5^{\prime} \mathrm{UTR}^{136,139}$ meaning that Nsp1 only inhibits translation of host genes. Some inhibitors (such as glycyrrhizic acid, lobaric acid, garcinolic acid, and tirilazad) that destroy the interaction between Nsp1 and SL1 are expected to be effective in COVID-19 treatment. $^{139}$

Nsp2 protein

Nsp2 is an endosome-associated protein with unknown specific functions. It interacts with multiple host proteins (such as Prohibitin 1[PHB1], PHB2, and actin-nucleation-promoting WASH protein) $^{140-142}$ and participates in biological processes such as viral replication, host immune regulation, mitochondrial biogenesis, and endosomal transport. ${ }^{143,144} \mathrm{Nsp} 2$ is therefore a promising novel target for the treatment of COVID-19.

SARS-CoV-2 Nsp2 comprises 638 amino acids, and its full-length structure has been obtained using cryo-electron microscopy in conjunction with AlphaFold2 structural prediction ${ }^{141}$ (Fig. 4b). The $\mathrm{N}$-terminus of Nsp2 (residues 1 to 276) consists of ten a-helices, fourteen $\beta$-strands, and three classic zinc-finger $(\mathrm{ZnF})$ structures: $\mathrm{C} 2 \mathrm{H} 2 \mathrm{ZnF}, \mathrm{C} 4 \mathrm{ZnF}$, and $\mathrm{C} 2 \mathrm{HC} \mathrm{ZnF}^{145}$ The residual structure of Nsp2 (residues 277-635) is relatively simple. Its carbon terminus consists of only $14 \beta$-strands, some of which form highly disordered loops, and one $3_{10}$ helix. The middle region contains three $\beta$-strands and nine $a$-helices. The Nsp2 protein binds nucleic acids nonspecifically, ${ }^{141,145}$ with the binding site being either $\mathrm{ZnFs}^{141}$ or the positively charged region on the surface of Nsp2; ${ }^{145}$ the specific identity of the binding site is controversial.

There are currently no verified inhibitors of Nsp2. Some potential Nsp2 inhibitors are derived from molecular docking. For example, nigellidine, ${ }^{146}$ an indazole-alkaloid, may bind to the entry pocket of Nsp2. It forms a hydrogen bond with Cys240 of Nsp2 (3.26 $\AA$ ), and thus occupies Nsp2 entry channel formed by several residues (Leu169, Val126, Trp243, Ala127, Cys132, Thr256, Gly257, Tyr242, and Val157). ${ }^{146}$ Notably, the increased transmissibility and pathogenicity of some SARS-CoV-2 variants are closely related to Nsp2 mutations, such as T85I of Nsp2 in the B.1.526/ B.1.427/B.1.429 variant. ${ }^{147-149}$ The presumed explanation is that mutations interfere with the interaction between Nsp2 and host proteins, ${ }^{141}$ ultimately affecting virulence. The immunogenicity of Nsp2 can be applied to the development of inactive or live attenuated virus vaccine.

\section{Nsp3 protein}

Nsp3 (also called papain-like protease, PLpro) is the largest membrane-associated cysteine protease produced by coronaviruses; it recognizes the tetrapeptide LXGG-motif and hydrolyzes viral polyprotein $\mathrm{pp} 1 \mathrm{a}$ to generate three nonstructural proteins (Nsp1, Nsp2, and Nsp3). ${ }^{150}$ A versatile protein, PLpro not only hydrolyzes ubiquitin and ubiquitin-like interferon-stimulated gene 15 protein (ISG15) but is also involved in post-translational modification of host proteins (de-ubiquitination and de-ISGylation). This role allows it to interfere with the host immune response, especially the interferon and NF-KB pathways. ${ }^{151-156}$ In summary, Nsp3 plays a critical role in viral reproduction and suppressing the host immune response, and is, therefore, an attractive drug target for the treatment of COVID-19.

SARS-CoV-2 Nsp3 consists of 10 domains, including the ubiquitin-like domain 1 (Ubl1, residues 1-108), hypervariable region (HVR, residues 109-206), macrodomain I (Mac1 or X, residues 207-386), "SARS-unique domain" (SUD; composed of three subdomains: macrodomain II [Mac2, residues 387-548], macrodomain III [Mac3, residues 549-676], and domain preceding Ubl2 and PL2pro [DPUP, residues 677-745]), ubiquitin-like domain 2 (Ubl2, residues 746-805), papain-like protease (PLpro, residues 806-1058), nucleic acid-binding domain (NBD, residues 1059-1200), marker domain (MD, residues 1201-1340), transmembrane regions (TM, residues 1341-1567), and the $\mathrm{Y}$ domain (residues 1568-1945) ${ }^{157-159}$ (Fig. 4c). The Ubl1, Mac1, and PLpro domains are discussed below in detail.

The core region of the Ubl1 domain (residues 18-109) folds into a canonical ubiquitin-like shape ( $\left.\beta 1-\alpha 1-\beta 2-\alpha 2-3_{10}-\beta 3-\beta 4\right)$ (PDB ID 7KAG), which resembles the structure of human ubiquitin (Ub) and two ubiquitin-like domains in human and mouse interferonstimulated gene 15 (hISG15 and mISG15). ${ }^{157}$ Ubiquitin-like modules are often involved in protein-protein interactions to regulate various biological processes. ${ }^{91,160}$ Distinct from Ubl2, the function of which is uncertain, Ubl1 specifically binds ssRNA with AUA patterns and interacts with the $\mathrm{N}$ protein. ${ }^{161}$ The latter interaction is essential for viral replication and pathogenicity. ${ }^{91}$ The interface regions of the Ubl1-N complex involve acidic residues of the Ubl1 helix $a 2$ and the SR-rich region of the $N$ protein. $^{91,162,163}$

In the processes of viral infection, the Mac1 domain counteracts host-mediated antiviral adenosine diphosphate-ribosylation signaling via its ADP-ribosyl hydrolase activity. ${ }^{164,165}$ Accordingly, catalytic null mutations of the Mac1 domain render viruses nonpathogenic, ${ }^{164-166}$ and the Mac1 domain is a promising drug target for disrupting the viral life cycle. The Mac1 domain adopts a conserved three-layered $\alpha / \beta / \alpha$ sandwich fold, in which there is a 
a

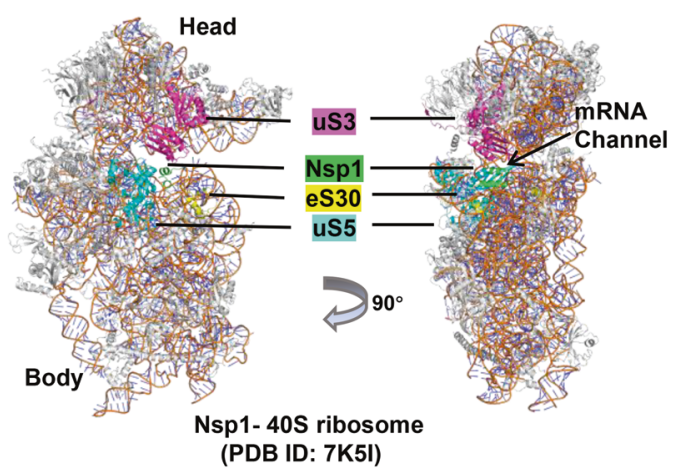

b

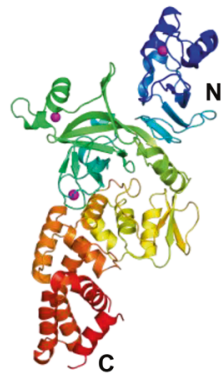

Nsp2

(PDB ID: 7MSX)

C

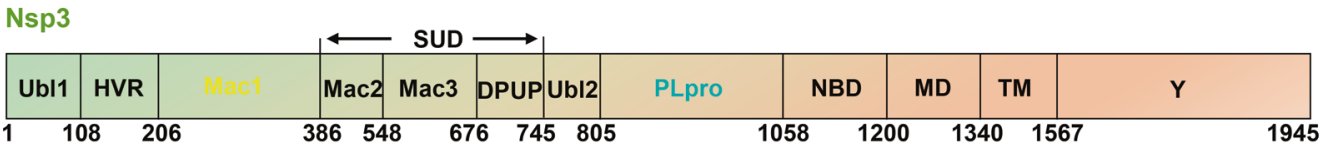

d

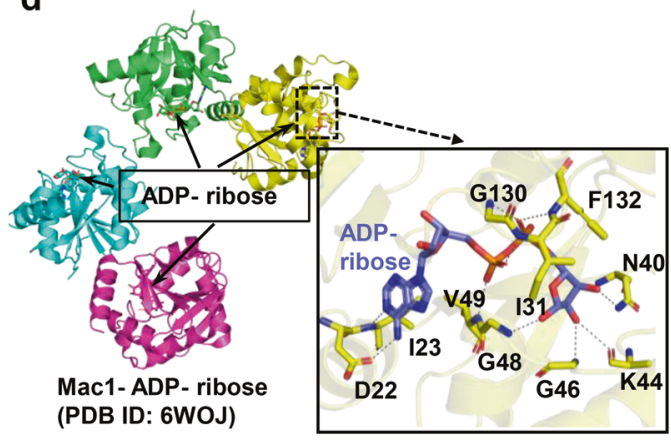

e

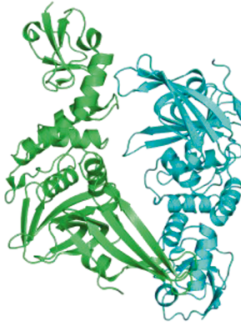

PLpro

(PDB ID: 7D7K) f

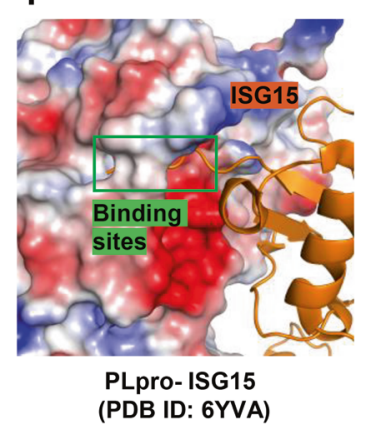

h

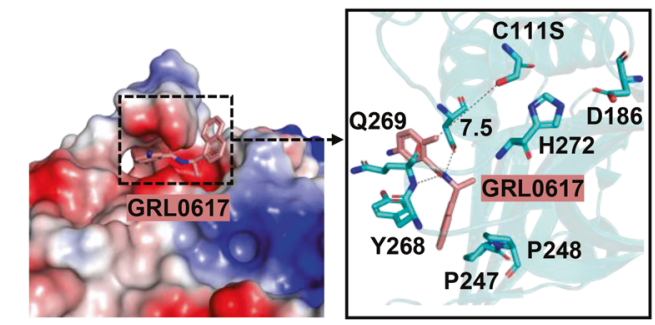

PLpro-GRL0617

(PDB ID: 7CJM)
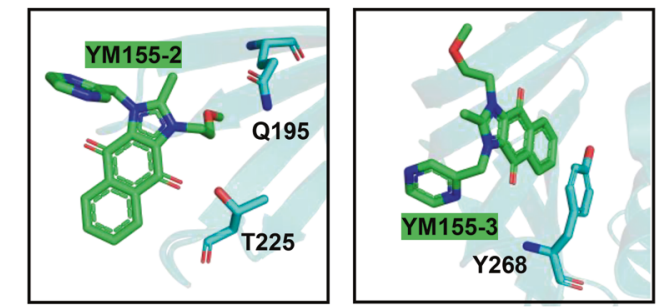

PLpro- ISG15

(PDB ID: 6YVA)

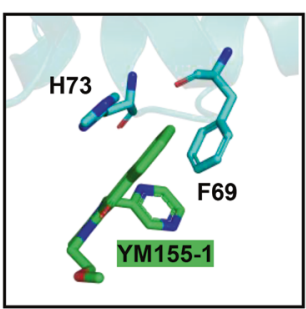

PLpro-YM155

(PDB ID: 7D7L)

g

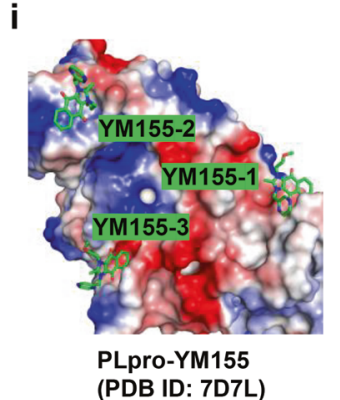

Fig. 4 Structures of Nsp1 /Nsp2 /Nsp3 and their inhibitors. a Structure of Nsp1 in complex with $40 \mathrm{~S}$ ribosome. Nsp1 binds at the mRNA channel in the cleft between the head and body of the $40 \mathrm{~S}$ ribosome. b Overall structure of Nsp2 protein. c Schematic representation of Nsp3 protein's domain. d Structures of Mac1 domain in complex with ADP ribose. e, f Structure of apo PLpro and its complex with interferonstimulated gene 15 protein (ISG15), respectively. g-i Structures of PLpro in complex with different inhibitors (VIR251, GRL-0617, and YM155). The PLpro is illustrated with electrostatic surface 
central seven-stranded $\beta$-sheet ( $\beta 1-\beta 2-\beta 7-\beta 6-\beta 3-\beta 5-\beta 4)$, and six $\alpha-$ helices are located on the outside. ${ }^{157,167}$ The Mac1 domain contains four substrate-binding pockets, namely the adeninebinding, distal ribose-binding, diphosphate-binding, and proximal ribose-binding sites. In the Mac1 domain-substrate (ADP-ribose [ADPr]) complex (Fig. 4d), ADPr is located in a cleft at the top of the central $\beta$-sheet. ${ }^{167,168}$ The adenine moiety is in a mostly hydrophobic environment, and its $\mathrm{N} 6$ and N1 atoms form hydrogen bonds with Asp22 and lle23, respectively. The proximal ribose ring is stabilized in the pocket by several hydrophobic interactions with Phe132/lle13, and hydrogen bonds with Gly46/ Gly48/Asn40. The $\alpha-/ \beta$-phosphate group is located in a narrow channel formed by loops $\beta 3-\alpha 2$ and $\beta 6-\alpha 5$, and accepts hydrogen bonds from Val49 and Gly130/Phe132, respectively. ${ }^{167,169}$ The distal ribose ring only participates in water-mediated hydrogen bonds with Leu126, Ala154, and Asp157. Asp22 and Asn40 appear to fix the two ends of ADPr. PDD00017273 is the only wellcharacterized inhibitor of macrodomain-type (ADP-ribosyl) hydrolase. ${ }^{16,170}$ Some small fragments that bind to the Mac1 domain have been identified using a combination of computational and structural analysis. The screened inhibitors occupy different sites in the Mac1 domain; these include the four substrate-binding sites mentioned above and the oxyanion subsite (adjacent to the adenine subsite and formed by Phe156/Asp157). ${ }^{164,171,172}$

PLpro has proteolytic, deubiquitinating (DUB), and delSGylating activity. ${ }^{173}$ The overall structure resembles a cellular DUB protein from herpesvirus (Fig. 4e), which folds into a right-handed shape composed of five domains: a palm domain, a thumb domain, a finger domain, the $\mathrm{N}$-terminal ubiquitin-like domain (UBL), and the C-terminal ubiquitin-specific protease domain (USP). ${ }^{174}$ SARS-CoV2 PLpro contains a narrow substrate-binding channel located at the interface of the thumb and palm domains ${ }^{175}$ (Fig. 4f). Trp106 and Asn 109 are proposed to form the oxyanion hole of SARS-CoV2 PLpro, which contributes to the stabilization of the oxyanion transition state of peptide hydrolysis. ${ }^{157,173,176,177}$ The protease activity of PLpro is in the monomer form, and its activity is regulated by the catalytic triad (Cys111-His272-Asp286), ${ }^{178}$ the zinc-binding structure located in the finger domain, ${ }^{179}$ and a unique gate (Leu-X-Gly-Gly) near the active center. ${ }^{180}$ Substrate access to the active site of PLpro is regulated by the flexible blocking loops 2 (BL2). ${ }^{157,175}$

Several representative inhibitors of PLpro are described here to illustrate their inhibitory mechanisms. (1) Inhibitors that target the active site, such as VIR251, a covalent peptide-mimetic inhibitor with a vinylmethylester (VME) warhead. ${ }^{173}$ This effectively inhibits SARS-CoV and SARS-CoV-2 PLpro activity. ${ }^{173}$ In the SARS-CoV-2 PLpro/VIR251 complex (Fig. 4g), P1-P4 amino acids of VIR251 insert into the S1-S4 pocket of PLpro adjacent to the active site. The GlyVME (P1) warhead forms a thioether bond with catalytic Cys 111 there, and a number of polar and hydrogen bonds (engaged by the P1-P3 positions) and hydrophobic interactions (engaged by the P4 position) mediate the stability of the complex. ${ }^{173}$ (2) Inhibitors that target the USP domain, such as GRL-0617, a naphthalene-based non-covalent inhibitor. ${ }^{151,180}$ In

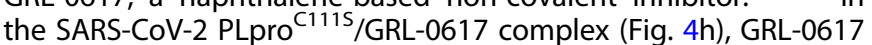
resides in a cleft distant from the catalytic triad with a minimum distance of $7.5 \AA$ to $\mathrm{S} 111$, and the side chains of Y268/Q269 in the BL2 loop undergo a large shift to better accommodate the compound. ${ }^{174}$ It has been confirmed that the interaction between GRL-0617 and PLpro blocks the C-terminus binding of ISG15 to PLpro. ${ }^{151,174}$ (3) Inhibitors that target multiple sites. These include YM155, an imidazolium-based inhibitor of the antiapoptotic protein survivin. ${ }^{175,181}$ In the SARS-CoV-2 PLpro ${ }^{\text {C111S/YM155 }}$ complex (Fig. 4i), YM155 binds to three different sites on each PLpro molecule, including the substrate-binding pocket, the ISG15 binding site, and the zinc-finger motif. ${ }^{175}$ The interaction between YM155 and PLpro is stabilized by interaction networks including hydrophobic interaction, $\pi$-stacking interaction and hydrogen bonding. Ultimately, YM155 affects the activity of PLpro protease and blocks C-terminus binding of ISG15 to PLpro.

Predicted structure of Nsp4

SARS-CoV-2 Nsp4 has four transmembrane domains (TMD1 4) and a large luminal domain in the endoplasmic reticulum (ER) between TMD1 and TMD2 and a smaller luminal domain in the ER lumen between TMD3 and TMD4. ${ }^{182} \mathrm{Nsp} 4$ can cause visible changes to ER structure. ${ }^{183}$ So far, only the crystal structure of C-terminal domain of Nsp4 (Ct-Nsp4) is available. Eribulin and Suvorexant were as promising drug candidates to target $\mathrm{Ct}-\mathrm{Nsp} 4$ by screening the 1600 FDA-approved drugs using molecular docking. ${ }^{184}$ In this manuscript, the structure of full Nsp4 was predicted by the latest prediction tool Alphafold 2. As shown in Fig. $5 \mathrm{a}$, the structure of full Nsp4 consists of nine a-helix and several $\beta$-sheets.

\section{Nsp5 protein}

Nsp5 is also known as the main-protease (Mpro) or 3C-like protease (3CLpro). It hydrolyzes viral polyprotein $1 \mathrm{ab}$, recognizing more than eleven cleavage sites to produce Nsp4-Nsp16, ${ }^{185}$ thus playing an essential role in viral replication and the maturation of nonstructural proteins. The absence of a human homolog of $3 C$ pro makes this protein one of the most attractive drug targets. ${ }^{186}$ Moreover, 3CLpro is highly conserved among coronaviruses; ${ }^{187}$ summarizing the structural similarities between viruses will be conducive to the development of broad-spectrum antiviral reagents.

The structure of $3 C$ Lpro is a homodimer, which is required for its enzymatic activity. Each protomer contains an N-finger and chymotrypsin-like domain I, domain II, and domain III $^{187}$ (Fig. 5b). Domain I and domain II form an antiparallel $\beta$-barrel, and domain III assembles into a globular shape consisting of five a-helices. Domain III and the N-finger (residues 1-7) are involved in the dimerization of Mpro. 3CLpro has two substrate-binding sites, an oxyanion hole (composed of Gly143, Ser144, and Cys145), and a substrate-binding pocket. The latter consists of several subsites, including the deeply buried subsites s1 (F140, L141, $\mathrm{N} 142, \mathrm{H} 163$, and E166) and s2 (M49, Y54, H164, D187, and R188); a hydrophobic subsite known as s4 (including M165, L167, Q189, T190, and Q192); and several extended solvent-accessible subsites, s3 (E166), s5 (including T190, A191, and Q192), and s1' (H41, G143, $\mathrm{S} 144$, and C145). The catalytic dyad (Cys145-His41) is located in the $s 1^{\prime}$ subsite, ${ }^{188}$ in which the cysteine acts as a nucleophile and the histidine as a base and proton acceptor. ${ }^{187}$ Based on the Schechter-Berger nomenclature, the residue sites of protease substrates or inhibitors that bind to the subsites of 3CLpro are defined as $\mathrm{Pn}, \mathrm{P} 1, \mathrm{P}^{\prime}{ }^{\prime} . . . \mathrm{Pn}^{\prime}$. The residues at the $\mathrm{Pn}$ positions may vary between different coronaviruses. For example, the P1 position is often Gln, whereas the P4 position may be Tyr, Thr, Ser, Ala, or Pro. ${ }^{185,189}$

3CLpro inhibitors can be divided into two groups: peptidemimetic inhibitors and non-peptide small molecular inhibitors. ${ }^{189}$ The former is similar to the natural peptide substrate of 3CLpro, and it is also the most studied at present. A range of specific chemical warheads, such as Michael acceptors, aldehydes, and epoxy ketones have been introduced at the P1/P2/P3 position by specific modifications of residues to strengthen the inhibitory effect on protease activity, antiviral ability, plasma half-life, and solubility. ${ }^{189}$ Generally, these inhibitors have covalent bond interactions with cysteine in the catalytic dyad and form a covalent adduct, which results in covalent inhibition of 3CLpro. Several representative inhibitors and their mechanisms of action are discussed below.

(1) N3 is a broad-spectrum antiviral peptidyl inhibitor with a Michael acceptor warhead. ${ }^{190}$ In the 3 CLpro/N3 complex (Fig. 5c), $\mathrm{N} 3$ is embedded in the substrate-binding pocket of 3CLpro in the form of an antiparallel sheet. ${ }^{186}$ It not only forms covalent bonds 
a

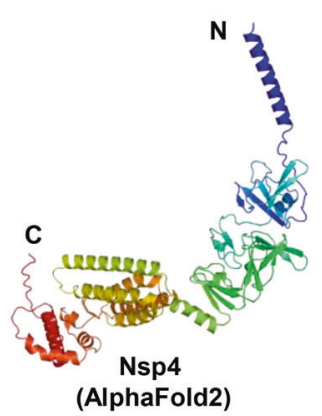

d

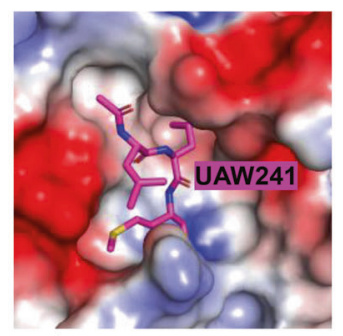

3CLpro-UAW241 inhibitor (PDB ID: 6XA4) b

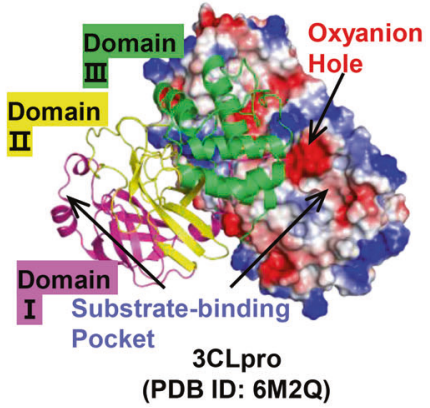

(PDB ID: 6M2Q)
C
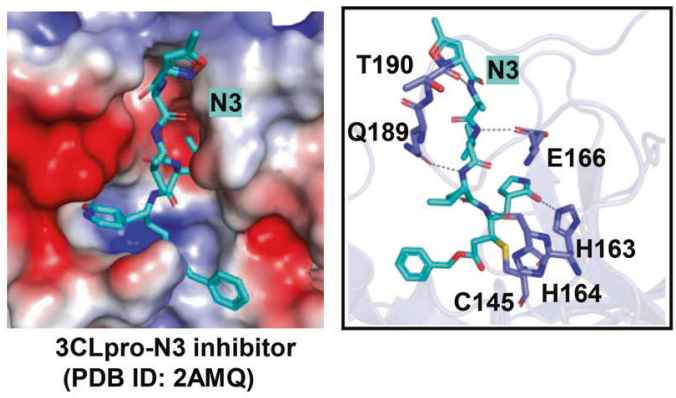

(PDB ID: 2AMQ)

e
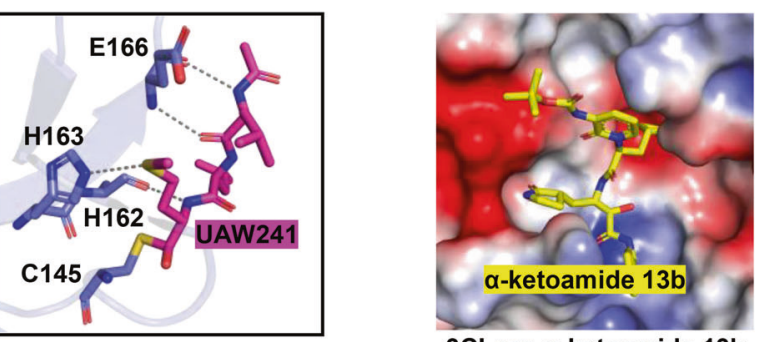

3CLpro-a-ketoamide 13b Inhibitor (PDB ID: 6Y2F)

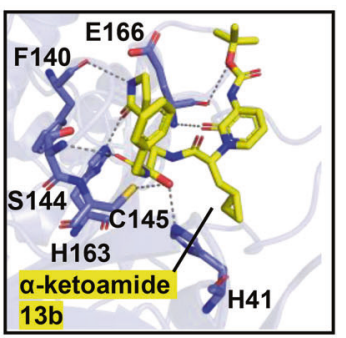

$13 \mathrm{~b}$ f

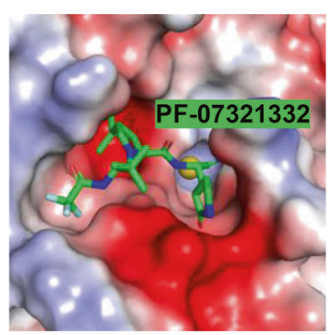

3CLpro-PF-07321332 Inhibitor

(PDB ID: 7VH8)

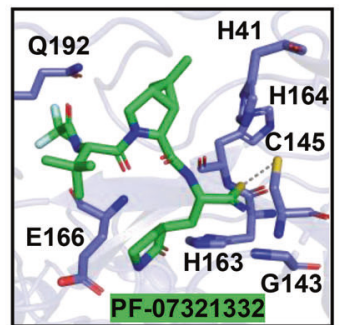

\section{tor}

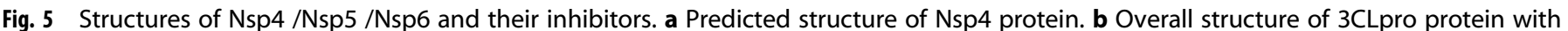

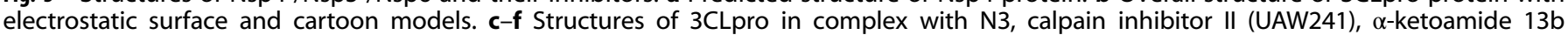
inhibitors, and PF-07321332, respectively. g Predicted structure of Nsp6 protein

with $C^{\beta}$ and $S^{\gamma}$ of $C 145$, but also forms various non-covalent bonds with residues in the substrate-binding pocket (including $\mathrm{H} 163$, H164, E166, Q189, and T190). ${ }^{186,189}$ (2) Calpain inhibitor II is a peptidyl inhibitor with an aldehyde warhead. ${ }^{189}$ In the 3CLpro/ Calpain inhibitor II (UAW241) complex (Fig. 5d), the P1-Met of Calpain inhibitor II forms a weak hydrogen bond with His163, occupying the s1 subsite. ${ }^{189,191}$ And other interactions occur between UAW241 and H162, E166, and C145. (3) a-ketoamide 13b is a broad-spectrum peptide-mimetic inhibitor of 3CLpro. ${ }^{192}$ In the 3CLpro/a-ketoamide complex (Fig. 5e), the a-keto warhead forms two covalent bonds with the Cys145-His41 catalytic dyad. ${ }^{187}$ This is distinct from other peptidyl inhibitors, which only have one covalent bond interaction with the catalytic center. ${ }^{187,189}$ (4) Oral antiviral PAXLOVID (Pfizer) is a mix of the antiviral PF-07321332 combined with a low concentration of Ritonavir which is an antiretroviral typically used against HIV, and have been shown to significantly reduce COVID-19 deaths in clinical trials. ${ }^{193}$ In the 3CLpro/PF-07321332 complex (Fig. 5f), the P1' nitrile warhead of PF-07321332 forms a reversible covalent thioimidate adduct with
Cys145 in the Cys145-His41 catalytic dyad, and therefore occupies the oxyanion hole of 3 CLpro. ${ }^{194}$ Certainly, other groups of PF07321332, including lactam ring, DMCP, and trifluoroacetyl group also participate in the stability of the complex. Small molecule inhibitors of 3CLpro are generally identified with a highthroughput screening of small molecule libraries, and their inhibitory mechanisms are slightly different from peptidyl inhibitors. An example is the benzotriazole-based inhibitor ribavirin, ${ }^{191,195}$ which is a covalent inhibitor.

Predicted structure of Nsp6

SARS-CoV-2 Nsp6 is a transmembrane protein and possesses eight transmembrane domains. Nsp6 expression inhibited the formation of a hybrid pre-autophagosomal structure (HyPAS). ${ }^{196}$ Moreover, Nsp6 can strongly blocked MAVS (mitochondrial antiviral signaling protein)-induced interferon $\beta$ production and binds TANK binding kinase1 (TBK1) to suppress interferon regulatory factor 3 (IRF3). ${ }^{197,198}$ Nsp6 also interacts with the sigma-1 receptor, which is considered an effective candidate host protein for host-based 
14

repurposing approaches to treat COVID-19 patients. $^{199}$ So far, there is no available structural information for Nsp6. The predicted overall three-dimensional structure of NSP6 consists of 14 ahelices, a C-terminal, two antiparallel $\beta$-strands, and 16 turns (Fig. $5 \mathrm{~g}$ ). The agonist of sigma receptors dextromethorphan binding leads to overall destabilization of Nsp6. ${ }^{200}$

\section{Nsp7 and Nsp8 protein}

Nsp7, Nsp8, and Nsp12 are the core components of the coronavirus replication machinery, in which Nsp12 acts as the RNA-dependent RNA polymerase (RdRp) and Nsp7/Nsp8 function as cofactors of Nsp12, possess primase activity, ${ }^{201,202}$ and mediate RdRp activity. ${ }^{203} \mathrm{Nsp7}$ and Nsp8 are highly conserved among coronaviruses, ${ }^{204,205}$ and further study of their structure and function will be conducive to the development of broad-spectrum inhibitors.

Nsp7 consists of 83 amino acids with only four a-helices (a1-a4) comprising its structure. ${ }^{204,205}$ In contrast to the relatively stable structure of $a 2 / a 3, a 1 / a 4$ adapts different lengths, positions, and relative orientations in various environments, and this variability plays an essential role in the assembly of the Nsp7-Nsp8 complex. $^{205}$ Nsp8 consists of 198 amino acids, including a long helical N-terminal domain (residues 1 to 77) and a conserved C-terminal domain (residues 78-198) (Fig. 6a). The N-terminal domain of Nsp8 is highly flexible and prone to proteolysis during the process of crystallization. ${ }^{204,206}$ Another feature of this domain is a positive electrostatic surface that may be used to bind viral RNA. $^{207,208}$ The C-terminus of Nsp8 comprises five a-helices and one four-stranded antiparallel $\beta$-sheet, and its topology resembles a golf club. ${ }^{209}$ The SARS-CoV-2 Nsp7-Nsp8 complex forms a hetero-tetramer [PDB ID 7JLT and 7DCD] ${ }^{204,206}$ (Fig. 6b), in contrast to the hexadecameric structure of SARS-CoV Nsp7-Nsp8. ${ }^{210}$ Two types of interfaces exist in the Nsp7-Nsp8 complex that synergistically mediates the stability of dimerization and tetramerization. ${ }^{204}$ Notably, both interfaces also mediate the dynamic assembly of the Nsp7-Nsp8-Nsp12 complex, and a large proportion of the residues located on the two interfaces are highly conserved among coronaviruses. ${ }^{204}$

The structural details of the Nsp7-Nsp8 complex reveal possibilities for the development of allosteric inhibitors that specifically inhibit RdRp activity. ${ }^{204}$ Distinct from nucleotide analogs that directly target RdRp, allosteric inhibitors interfere with the activity of RdRp machinery by disrupting the assembly of Nsp7-Nsp8-Nsp12. For example, the Nsp7 ${ }^{\mathrm{N} 37 \mathrm{~V}}$ mutant, which affects the stability and activity of Nsp7-Nsp8-Nsp12 complex, could be exogenously introduced into the infected cells to inhibit viral proliferation. ${ }^{204}$

\section{Nsp9 protein}

SARS-CoV-2 Nsp9 shares $97 \%$ sequence identity with that of SARSCoV Nsp9, both of which belong to the oligonucleotide/ oligosaccharide binding superfamily (OB-fold) and specifically bind to single-stranded DNA and RNA oligonucleotides. ${ }^{211} \mathrm{Nsp} 9$ is involved in the formation of the replication and transcription complex (RTC) and plays a significant role in viral replication. ${ }^{190,207,212}$ The crystal structure of SARS-CoV-2 Nsp9 is a homodimer, ${ }^{213}$ and the arrangement of protomers is conserved with respect to other coronaviruses. The core comprises a seven $\beta$-strand enclosed $\beta$-barrel, and the $C$-terminus has a flexible $\alpha$ helix (residues 96-109) that contains the conserved protein-protein interaction motif (GXXXG). Two combination modes, the helix interface and the sheet interface, contribute to the stability of the dimer. The former is formed by two GXXXG motifs with strong van der Waals interactions, whereas the latter includes $\beta 5$ and connection loops. ${ }^{214,215}$

The mechanism of RNA binding with Nsp9 is still unclear. A previous study identified the possible RNA-binding sites (Phe40, Val41, and Ile91) by observing the chemical perturbation of Nsp9 titrated with ssRNA using nuclear magnetic resonance (NMR) titration assays. ${ }^{216}$ Subsequently, targeted substrates that bind to these sites (or adjacent to these sites) were screened from a small fragment library; the uracil-analog FR6, which has a weak affinity with Nsp9, was obtained. ${ }^{216}$ In the Nsp9/FR6 complex, a tetrameric $\pi-\pi$ stacking between the pyrimidinedione ring of FR6 and the aromatic ring of Phe40 induces a hexameric form of Nsp9 (a "trimer of dimers"). ${ }^{216}$ Changes in the oligomerization state may alter RNA entry channels and thus affect RNA binding. In addition to the inhibitors of Nsp9-RNA binding described above, other inhibitors have been identified that target the Nsp9 GXXXG motif to disrupt the dimer interface, affecting RNA binding and viral proliferation. $^{213,217}$ Finally, several potential inhibitors (such as imidazolium salts ${ }^{218}$ and teicoplanin ${ }^{219}$ ) have also been identified through molecular docking, but the inhibition mechanisms are unknown.

Nsp10 protein

Nsp10, a protein unique to viruses, ${ }^{125}$ plays a crucial role in viral mRNA capping via methylation, a process that promotes stability and effective translation of viral RNA. ${ }^{220,221}$ Four sequential enzymatic reactions are involved in coronavirus RNA capping. Initially, RNA 5'-triphosphatase in Nsp13 hydrolyzes nascent RNA to produce pp-RNA; an unknown guanylyl-transferase (GTPase, possibly Nsp12) ${ }^{222}$ then hydrolyzes GTP and transfers the product (GMP) to pp-RNA to form Gppp-RNA; in the subsequent reaction, N7-methyltransferse (N7-MTase) methylates Gppp-RNA to create Cap-0 ( $\left.\mathrm{m} \mathrm{GpppA} \mathrm{A}_{1}\right)$; in the final step, the ribose 2'-O of the first nucleotide in Cap-0 is methylated by Nsp16, resulting in the formation of Cap-1 $\left(\mathrm{m}_{7 \mathrm{GpppA}} \mathrm{Am}_{\mathrm{m}}\right)^{223,224}$ Throughout the process, Nsp10 specifically binds to and stimulates the N7-MTase and the 2'-O-methlytransferase (2'-O-MTase) activity of Nsp14 and Nsp16, respectively, 223,224 which provides the molecular connector between proofreading and capping activities. ${ }^{225}$ Therefore, a scheme is proposed to interfere with the activity of Nsp14 and Nsp16 with the peptide derivatives of Nsp10. ${ }^{226,227}$ For example, a peptide (defined as K29) from SARS-CoV Nsp10 (resides 68 to 96) could significantly inhibit the activity of Nsp16. ${ }^{226}$ The same approach could be applied to screen peptide inhibitors against SARS-CoV-2. In this section, we focus on the structure of Nsp10; the structure of the Nsp10-Nsp14 and Nsp10-Nsp16 complexes will be described in the following chapters.

SARS-CoV-2 Nsp10 and SARS-CoV Nsp10 have nearly identical sequences (99\%) and structures, ${ }^{125}$ both of which comprise five ahelices, an antiparallel $\beta$-sheet, and two zinc-finger structures (ZnF1 and ZnF2) (PDB ID 6ZPE). ${ }^{125} \mathrm{ZnF1}$ is coordinated by three cysteines and one histidine, whereas $\mathrm{ZnF2}$ is coordinated by four cysteines. The zinc-finger structure enables Nsp10 to play a significant role in viral RNA synthesis. 228

\section{Nsp12 protein}

Coronaviruses utilize the RTC to complete viral genome replication and mRNA transcription. ${ }^{9} \mathrm{Nsp} 12$, which is highly conserved among coronaviruses, is the core component of RTC. Nsp12 assembles with other nonstructural proteins to form various architectures ${ }^{229}$ such as the central RTC (C-RTC, Nsp12-Nsp7-Nsp8) ${ }^{230}$ (Fig. 6c, d), the elongation RTC (E-RTC, Nsp12-Nsp7-Nsp8-Nsp13) ${ }^{222}$ (Fig. 6e), the Cap $(-1)^{\prime}$-RTC (Nsp12-Nsp7-Nsp8-Nsp13-Nsp9) ${ }^{222}$ (Fig. 6f), the Cap (0)-RTC (Nsp12-Nsp7-Nsp8-Nsp13-Nsp9-Nsp14-Nsp10) ${ }^{231}$ (Fig. 6g) and the Cap (1)-RTC (Nsp12-Nsp7-Nsp8-Nsp9-Nsp16Nsp10). These architectures play a pivotal role in viral proliferation and host immune regulation. Therefore, further understanding of the structure and catalytic mechanism of Nsp12 could accelerate the development of broad-spectrum antiviral agents.

SARS-CoV-2 Nsp12 is composed of 932 amino acids, consisting of the N-terminal nidovirus RdRp-associated nucleotidyl-transferase domain (RiRAN, residues 117-250), ${ }^{232}$ the interface domain (residues 251-365), and the RdRp domain. The latter is subdivided 
a

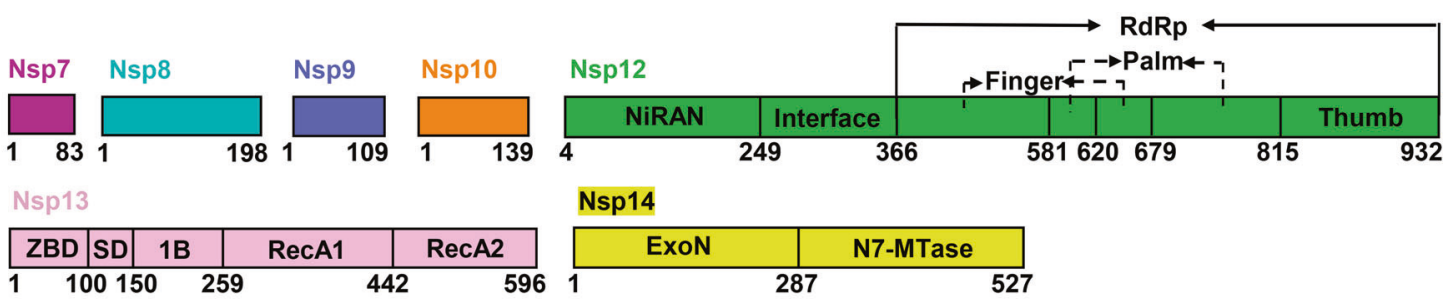

b

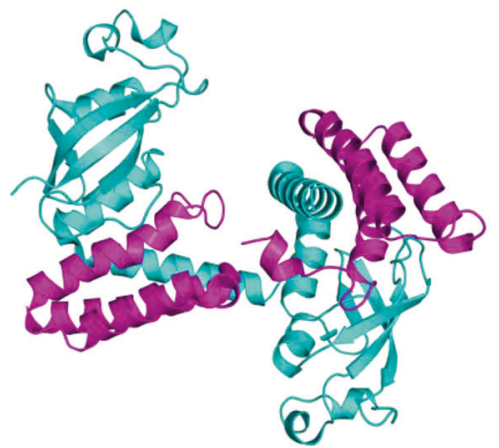

Nsp7-Nsp8 complex (PDB ID: 6XIP)

e

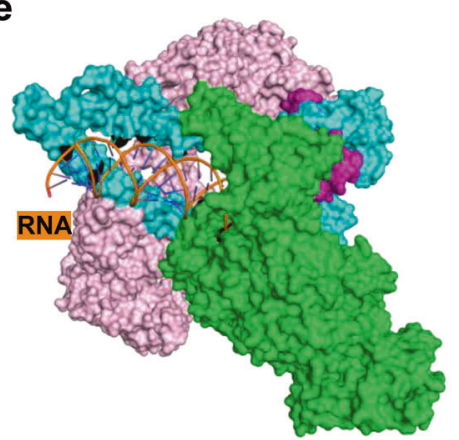

E-RTC complex (PDB ID: 7CXM)
C

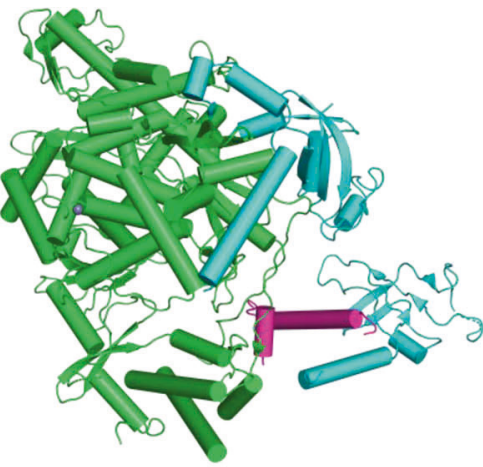

Nsp7-Nsp8-Nsp12 complex (PDB ID: 7BV1) d

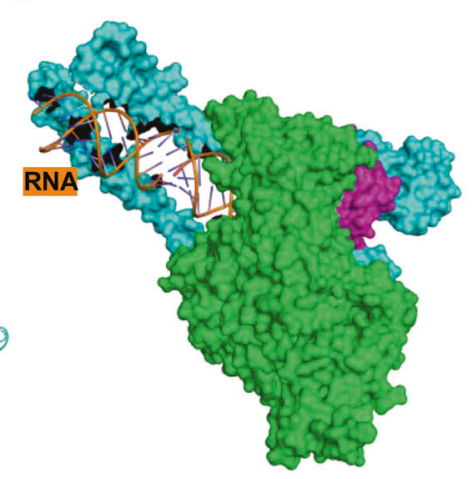

C-RTC complex (PDB ID: 6YYT)
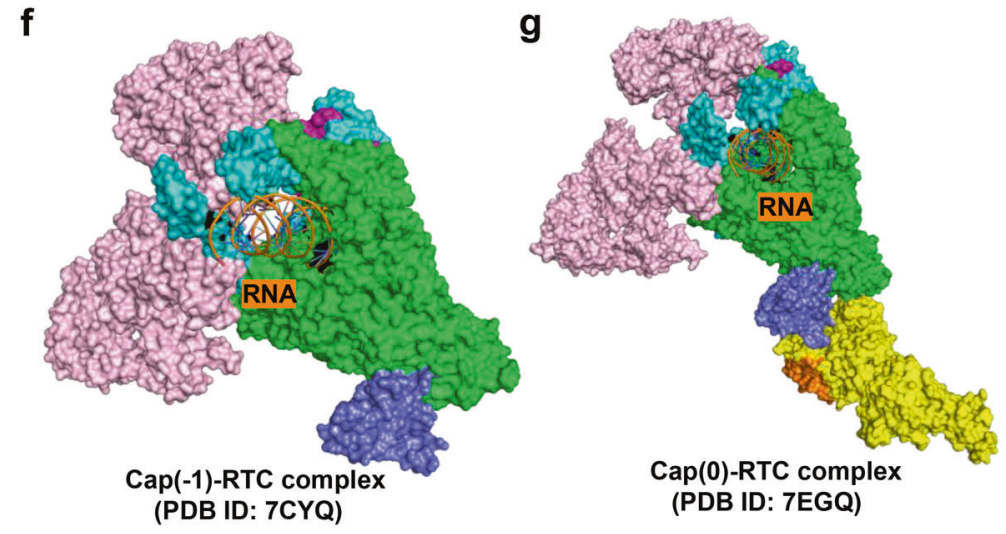

h
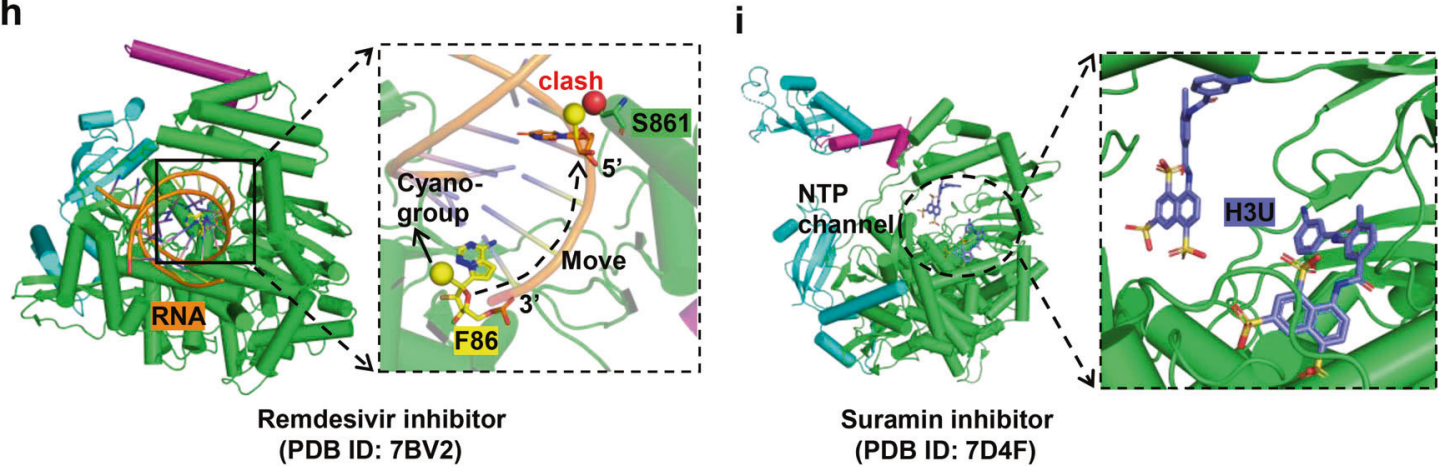

Fig. 6 Structures of SARS-CoV-2 RTC complexes and cognate inhibitors. a Schematic representations of Nsp7, Nsp8, Nsp9, Nsp10, Nsp12, Nsp13, and Nsp14 proteins organizations. b-g Structures of Nsp7-Nsp8, Nsp7-Nsp8-Nsp12, the central RTC (C-RTC, Nsp12-Nsp7- Nsp8), the elongation RTC (E-RTC, Nsp12-Nsp7-Nsp8-Nsp13), cap (-1)-RTC (Nsp12-Nsp7-Nsp8-Nsp13-Nsp9), and cap (0)-RTC (Nsp12-Nsp7-Nsp8- Nsp13Nsp9-Nsp14-Nsp10) complexes, respectively. $\mathbf{h}$, i Structures of C-RTC with bound inhibitors Remdesivir (F86) can bind to the RNA strand. The schematic of the inhibition state is shown on the right $(\mathbf{h})$. Two suramin $(\mathrm{H} 3 \mathrm{U})$ molecules occupy the catalytic cavity (i) 
into the finger subdomain (residues 367-581 and 621-679), the palm subdomain (residues 582-620 and 680-815), and the thumb subdomain (residues 816-932). ${ }^{233}$ The single RdRp domain folds into a right-hand shape that resembles other RNA polymerases, ${ }^{233}$ whereas the heterotrimeric complex formed by Nsp12, Nsp7, and Nsp8 (PDB ID 6YYT and 7BV2) is packed into a stable closed conformation. ${ }^{203,234}$ Two conserved zinc-finger structures in this complex (ZnF1 and ZnF2, coordinated by H295/C301/C306/C310 and $\mathrm{C} 487 / \mathrm{H} 642 / \mathrm{C} 645 / \mathrm{C} 646)$ contribute to the structural integrity of RdRp. ${ }^{234}$ Seven conserved active sites (motifs $A-G$ ) are located throughout the RdRp domain, in the palm (motifs $A, B, C, D, E$ ) and finger (motifs $F$ and $G$ ) subdomains, which direct and stabilize the RNA template-product duplex by interacting with RNA templateand primer-strands. ${ }^{234}$ In addition, there is a positively charged 'sliding poles' structure formed by two copies of Nsp8 N-terminal domain in the complex, which is verified to accommodate the RNA duplex outside the active sites. ${ }^{203}$

Presently, numerous nucleoside analog drugs are readily available, such as ribavirin and favipiravir (guanine analogs), 235,236 sofosbuvir (uridine analog), ${ }^{237}$ and molnupiravir (also named MK4482 or EIDD-2801), ${ }^{238}$ remdesivir, and galidesivir (adenosine analogs). ${ }^{239} \mathrm{Nsp} 12$ is considered a primary target for these. ${ }^{240}$ For example, remdesivir is a broad-spectrum antiviral agent and the first FDA-approved drug for the treatment of COVID-19. ${ }^{241,242}$ In the C-RTC/RNA/remdesivir complex (Fig. 6h), the remdesivirmonophosphate (RMP) is located at the catalytic active site of RdRp and covalently incorporated into the RNA primer strand at the +1 position. ${ }^{234}$ The RMP interacts with Nsp12 in a manner identical to adenosine monophosphate (AMP), but the steric clash between the cyano group of RMP and Ser861 of Nsp12 blocks RNA translocation after incorporation of three bases following RMP. ${ }^{234}$ This ultimately results in delayed chain termination. ${ }^{243,244}$ In addition to the above mechanism, another inhibition forms of remdesivir action was recently proposed, namely RNA templatedependent inhibition of RdRp. ${ }^{245}$ A promising orally available drug to treat COVID-19, molnupiravir is receiving more and more attention. The inhibition mechanism of RdRp by molnupiravir is different from remdesivir. In the process of RNA synthesis, the active form of molnupiravir, $\beta$-D- $\mathrm{N}^{4}$-hydroxycytidine (NHC) triphosphate (MTP), can form stable base pair with either $G$ or $A$ in the RdRp active center. ${ }^{246}$ Notably, these mis-incorporations would not lead to stalls RdRp like remdesivir, and this NHC-containing RNA product can also be used as an RNA template in a new round of RNA synthesis. ${ }^{246}$ So, molnupiravir can adopt a two-step mutagenesis mechanism to cause an "error catastrophe" during viral RNA replication. Certainly, the difference between molnupiravir and remdesivir in inhibiting RNA synthesis by RdRp also indicates that nucleoside analogs have diverse antiviral mechanisms.

There are several other drugs that target RdRp, such as suramin, ${ }^{247}$ a poly-sulfonated trypan blue derivative that effectively inhibits a variety of viruses including SARS-CoV- $2 .{ }^{26}$ In the RdRp-suramin complex (Fig. 6i), two suramin molecules occupy the catalytic cavity, which in turn blocks the RNA template-primer strand from binding to the active site and blocks NTP substrate entry into the catalytic site. ${ }^{248} \mathrm{Nsp} 12$ additionally has GTPase activity to catalyze the formation of GpppA, but Nsp9 specifically inhibits this activity. ${ }^{222}$ In the Nsp12/Nsp7/Nsp8/Nsp13/Nsp9 complex (PDB ID 7CYQ), the N-terminus of Nsp9 inserts into the catalytic center of Nsp12, interacting with the bound GDP. ${ }^{222}$ Therefore, peptides with similar properties to the N-terminus of Nsp9 could be designed to inhibit the activity of Nsp12. Guanine analogs can also be utilized to inhibit the GTPase activity of Nsp12 and interfere with mRNA capping. ${ }^{222}$

Nsp13 protein

Nsp13 has helicase and RNA 5'-triphosphatase activity, with which it participates in unwinding DNA or RNA during RNA replication in an ATP-dependent manner ${ }^{249,250}$ and in the first step of mRNA capping, ${ }^{251,252}$ respectively. Nsp13, therefore, has crucial roles in viral proliferation and can be regarded as a drug target for treating COVID-19. ${ }^{253}$

SARS-CoV-2 Nsp13 consists of 601 amino acids, including the $\mathrm{N}$-terminal zinc-binding domain (ZBD) (residues 1-100), a stalk domain (SD, residues 101-150), an inserted domain 1B (residues 151-259), and two helicase domains: RecA1 (residues 260-442) and RecA2 (residues 443-596). ${ }^{254,255}$ Nsp13 and the C-RTC (Nsp12Nsp7-Nsp8) form a stable complex, E-RTC (L) (Fig. 6e), and the helicase activity of Nsp13 is enhanced by the formation of this complex. ${ }^{207,208,256}$ Two Nsp13 protomers play different roles in the E-RTC complex (PDB ID 7CXM and 7CXN); ${ }^{208}$ Nsp13-1 stabilizes the structure of RTC by interacting with Nsp12 and Nsp8-1, whereas Nsp13-2 (with a larger conformational shift compared to the free state) provides an RNA-binding channel for the unpaired $5^{\prime}$ extension of RNA template. ${ }^{222}$ Residues in the Nsp13-2 RNAbinding channel that are involved in RNA recognition are highly conserved in coronaviruses. These include N361 in the RecA1 domain, S468/T532/D534 in the RecA2 domain, and R178/H230 in the $1 \mathrm{~B}$ domain. ${ }^{222,257}$ The paired portion of template-primer RNA is located in the pocket formed by Nsp8 and Nsp12. In the process of viral RNA replication, Nsp13 anchors the $5^{\prime}$ extension of template RNA and stimulates the RdRp backtracking that is a ubiquitous transcriptional regulatory mechanism. ${ }^{207,257-260}{\mathrm{~A} 3^{\prime}}^{\prime}$ end of primer-strand RNA containing mismatched nucleotides can be guided toward the RdRp NTP entry tunnel as a result of the RdRp backtracking capability, providing access for proofreading machinery (Nsp14/Nsp10). ${ }^{257,261}$ This action can allow nucleotide analog-based resistance. ${ }^{262}$ Therefore, inhibitors that interfere with the activity of $\mathrm{Nsp13}$ and RdRp backtracking should be administered in combination with nucleoside analogs to prevent drug resistance.

\section{Nsp14 protein}

As a dual-functional enzyme, Nsp14 has both $3^{\prime}-$ to- $5^{\prime}$ exoribonuclease (ExON) and N7-MTase activities, which are responsible for nascent RNA proofreading and mRNA capping during viral RNA replication, respectively. ${ }^{224,263,264}$ The significance of Nsp14 in high-fidelity replication of viral RNA and host immune defense escape makes this protein an attractive target for antiviral treatment. ${ }^{265-267}$ Furthermore, Nsp14 is highly conserved among the coronaviruses, ${ }^{268}$ and drugs targeting this protein have the potential to be pan-inhibitors for other viruses.

SARS-CoV-2 Nsp14 consists of 527 amino acids, including the $\mathrm{N}$-terminal ExoN domain (residues 1-287) and the C-terminal N7MTase domain (residues 288-527). The activity of ExoN can be stimulated by the cofactor Nsp10 independent of the N7-MTase domain. 264,269,270 When ExoN forms the ExoN-Nsp10 complex, Nsp10 does not undergo a significant conformational change, ${ }^{270}$ and the complex structure resembles the Asp-Glu-Asp-Asp (DEDD)-type exonuclease. ${ }^{264,270}$ Both possess typical DED/Edh motifs (D90/E92/E191/H268/D273) ${ }^{271}$ and adopt similar topological folds (a central twisted $\beta$-sheet flanked by a-helices on either side). However, distinct from the DEDD-type exonuclease, Nsp14 contains two zinc-binding sites ( $\mathrm{ZnF1}$ and $\mathrm{ZnF} 2)$ that are located on both sides of the $\beta$-sheets and coordinated by C207/C210/ C226/H229 and H257/C261/H264/C279, respectively. These zincfinger structures are associated with the stability and enzymatic activity of ExoN. ${ }^{264}$ A convoluted loop (residues 288-301) joins the N7-MTase domain and the ExoN domain. In contrast to ExoN, N7MTase activity is independent of Nsp14-Nsp10 complex formation, ${ }^{272,273}$ and there are no interactions between the N7-MTase domain and Nsp10. The N7-MTase domain adopts a noncanonical MTase fold with a three-stranded $\beta$-sheet insertion and a peripheral zinc finger ( $\mathrm{ZnF3})$. $\mathrm{ZnF3}$, however, has a limited role in the activity of N7-MTase, and may instead be involved in protein-protein interactions. ${ }^{274}$ 
a

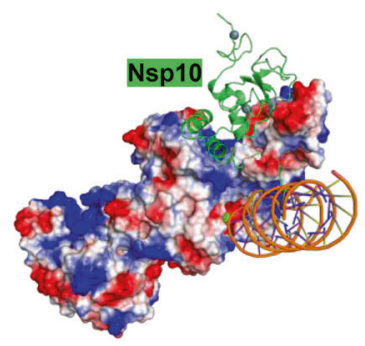

Nsp14-Nsp10-RNA

(PDB ID: 7NOC) b

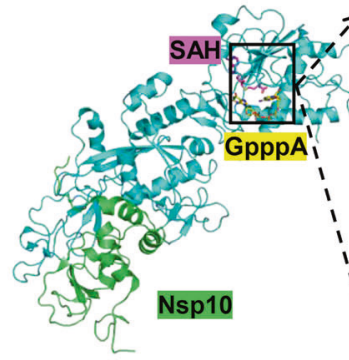

Nsp14-Nsp10-SAH-GpppA (PDB ID: 5C8S)

C

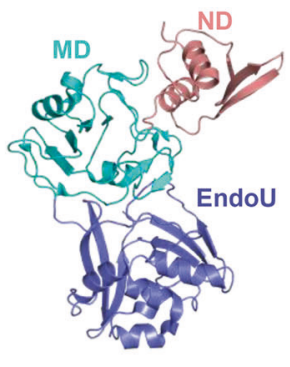

Nsp15

e
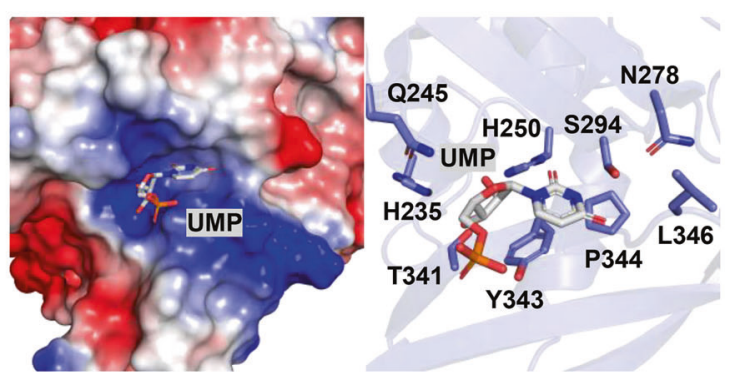

Nsp15-UMP (PDB ID: 6WLC)

g
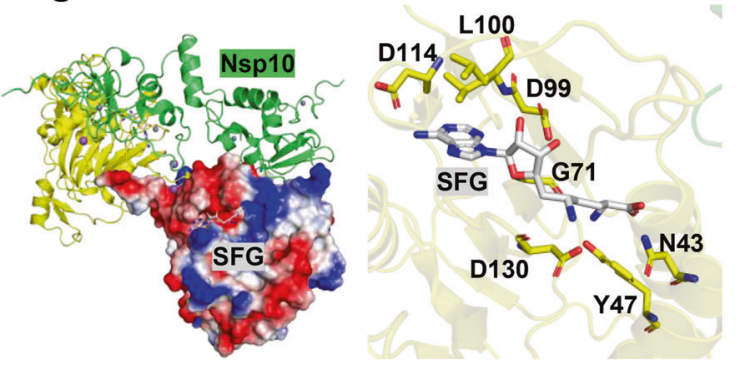

Nsp16-Nsp10-SFG inhibitor (PDB ID: 6WKQ)

Fig. 7 Structures of Nsp14 /Nsp15/Nsp16 and their inhibitors. a Cryo EM structure of the SARS-CoV-2 Nsp10-Nsp-14 RNA complex. Nsp14 is illustrated with electrostatic surface. Nsp10 is illustrated with cartoon in green. b The structure of the Nsp14-Nsp10 in complex with functional ligands S-adenosyl-L-homocysteine (SAH) and GpppA shown in sticks. c The overall structural of Nsp15. d Conformational changes between Nsp15 in pre- and post- cleavage states. e Structures of Nsp15 in complex with uridine-5'-monophosphate (UMP). $\mathbf{f}$ The structure of SARS-CoV2 Nsp16-Nsp10 in complex with RNA cap analogue (m7GpppA) and S-adenosyl methionine (SAM). The m7GpppA and SAM are shown in sticks. Nsp16 is indicated as electrostatic surface. $\mathbf{g}$ The structure of Nsp16-Nsp10 heterodimer in complex with sinefungin (SFG). SFG is shown in sticks. Nsp16 is illustrated with electrostatic surface and cartoon in yellow

Understanding the molecular mechanisms of proofreading and methylation will be beneficial in developing inhibitors of Nsp14. In the ternary complex of Nsp14-Nsp10-RNA (bearing a 3'-end mismatch) (Fig. 7a), only three base pairs (including a mismatched C-U pair at the $3^{\prime}$-end of the RNA) are located in a narrow pocket on the ExoN surface, and most of the RNA helix is in an unbounded or solvent-exposed state. ${ }^{275}$ This complex of RNA substrate and Nsp14 is determined by two key residues (H95 and P142), which restrict the depth of the substrate-binding pocket and may force the separation of primer-strand and templatestrand RNA. ${ }^{275}$ There are also two Mg-binding sites (Mg1 and Mg2) in the ternary complex, in contrast to the one Mg-binding site in the binary structure of Nsp14-Nsp10. ${ }^{264,270}$ One magnesium ion activates a water molecule for nucleophilic attack and the other stabilizes the $\mathrm{O}^{\prime}$ ' leaving group of $-1 \mathrm{C}_{\mathrm{p} .}{ }^{275}$ The methylation reaction mechanism of the N7-MTase can be determined from the structure of the Nsp14-Nsp10-SAH-G binding site of the substrate S-adenosyl methionine (SAM) is adjacent to the GpppA binding site (Fig. 7b). This combination may facilitate methyl transfer from donor to acceptor. ${ }^{264,274}$

As an essential exoribonuclease in coronavirus, Nsp14 removes both mis-incorporated nucleotides and nucleotide analogs from the nascent RNA, making viruses that encode it prone to develop nucleotide analog-based antiviral resistance. ${ }^{276,277}$ The combination of Nsp14 inhibitors and nucleotide analogs (such as remdesivir, ${ }^{278}$ sofosbuvir, ${ }^{279}$ and ribavirin) have the potential to resolve this issue. ${ }^{280} \mathrm{Nsp} 14$ inhibitors take multiple forms, as described below.

(1) $3^{\prime}$-deoxy nucleotide analogs inhibit the activity of ExoN. ${ }^{275}$ (2) $\mathrm{Zn}^{2+}$-ejecting agents, such as disulfiram and ebselen, 
synergistically inhibit Nsp14 activity via their three zinc-binding sites. ${ }^{281,282}$ (3) SAM competitive inhibitors and SAM analogs, such as S-adenosyl-homocysteine (SAH), sinefungin (SFG), and aurintricarboxylic acid (ATA) ${ }^{273,283}$ interfere with N7-Mase activity and subsequently impede $5^{\prime}$-end cap formation. ${ }^{284}$ In addition to these inhibitors, mutations in Nsp14 can lead to virus attenuation and induction of higher interferon response. Live attenuated virus vaccine development is, therefore, an option for this target in addition to the development of antibodies. ${ }^{285-287}$

\section{Nsp15 protein}

Nsp15 (also called EndoU) ${ }^{288}$ is a uridine-specific, $\mathrm{Mn}^{2+}$-dependent endoribonuclease that has functional characteristics and active sites similar to eukaryotic RNase. ${ }^{289,290}$ Coronavirus Nsp15 cleaves the 5'-polyuridine tracts in negative-strand RNA and prevents the activation of host pattern recognition receptor MDA5-mediated immune response. ${ }^{291-293}$ The crucial function and extreme conservation of Nsp15 in coronaviruses make it a promising target for COVID-19 treatment. $^{294}$

SARS-CoV-2 Nsp15 consists of 347 amino acids, including the $\mathrm{N}$-terminal domain (ND, residues 1-64), the middle domain (residues 65-182), and the C-terminal EndoU domain (residues 207-347). ${ }^{295}$ The overall structure of SARS-CoV-2 Nsp15 is a homohexamer with D3 symmetry ${ }^{289,295-297}$ (Fig. 7c). Several conserved residues in Nsp15 (including His235, His250, Lys290, Thr341, Trp333, Tyr343, Ser294, Gly248, Lys345, Val295, and GIn245) are involved in substrate specificity, nuclease activity, and oligomerization of EndoU. ${ }^{295,298}$ His235, His250, and Lys290 constitute the catalytic triad and utilize the general acid-base catalytic mechanism to complete the cleavage reaction. ${ }^{289,295-297}$ The EndoU domain has conformational variability, and the substrate uridine$5^{\prime}$-Monophosphate (UMP) restrains this dynamic. 295 Notably, allosteric regulation exists in the excision reaction of EndoU, ${ }^{297,299}$ and the base binding sites are different between RNA in the preand post-cleavage states ${ }^{297}$ (Fig. 7d, e). In the Nsp15/5'-UMP and Nsp15/AUF A complexes Ser294 forms two hydrogen bonds with $\mathrm{O} 2$ and N3 of the uracil base and Tyr343 forms $\pi-\pi$ stacking interactions with the ribose ring. ${ }^{289,300}$ Consequently, Ser294 and Tyr343 of Nsp15 may be responsible for base discrimination by EndoU. ${ }^{295,300}$ In the Nsp15/AU-3'P complex (PDB 7N06), Trp333 forms $\pi-\pi$ stacking interactions with uracil, but Ser294 no longer forms hydrogen bonds with uracil due to a conformational flip. ${ }^{297}$ The ND domain also plays an indispensable role in the function of Nsp15, participating in protein oligomerization and RNA binding. ${ }^{297,299}$

Some inhibitors targeting Nsp15 are uracil derivatives or modified oligonucleotides containing these derivatives, such as tipiracil or modified RNA with $2^{\prime}$-fluorine instead of $2^{\prime}-\mathrm{OH}$ on the uridine ribose. 289,301 Tipiracil effectively inhibits the activity of EndoU and binds in a manner similar to uridine in the Nsp15/ tipiracil complex. ${ }^{289,302}$ Compounds that disturb the stability of the hexamer conformation, and thus interfere with the activity of EndoU, could be screened as inhibitors of Nsp15. An attenuated live virus vaccine with Nsp15-defective SARS-CoV-2 has also been proposed. $^{292}$

\section{Nsp16 protein}

As mentioned above, Nsp16 is a $\mathrm{Mg}^{2+}$ and Nsp10-dependent 2'O-methyltransferase in coronaviruses that methylates the ribose 2 '-O of the first nucleotide in Cap-0 mRNA ( $\left.m 7 \mathrm{G}_{0 p p p} \mathrm{~A}_{1}-\mathrm{RNA}\right)$ to produce Cap-1 mRNA ( $\left.\mathrm{m} \mathrm{G}_{0 \mathrm{ppp}} \mathrm{A}_{1 \mathrm{~m}}-\mathrm{RNA}\right){ }^{303-306}$ In the Nsp16Nsp10-SAM-m7GopppA ${ }_{1}$ (or $\mathrm{m}^{7} \mathrm{G}_{0} \mathrm{pppA}_{1 \mathrm{~m}}$ ) complex (Fig. 7f), an Nsp16 protomer is anchored on the top of an Nsp10 promoter. ${ }^{307-}$ ${ }^{309}$ The structure of Nsp10 in the heterodimer does not undergo a significant conformational change, aside from the a1-helix, as previously reported. ${ }^{125,307,308}$ Nsp16 employs a Rossmann-like fold consisting of a centrally located twisted $\beta$-sheet of eight $\beta$-strands $(\beta 1-\beta 8-\beta 9-\beta 6-\beta 7-\beta 2-\beta 3-\beta 4-\beta 5)$ flanked by $\alpha$-helices and $\beta$-strands.
Various substrates of Nsp16 bind in different pockets on the surface of the protein. Cap is located in a positively charged pocket with intrinsic plasticity, in which Lys137 and Tyr30 form a partial enclosure to restrict the movement of Cap. ${ }^{308}$ SAM is located in a negatively charged pocket, and a number of interactions (including electrostatic, hydrophobic, and Van der Waals interactions) mediate the stability of SAM; Nsp10 also plays a fixed role in the SAM-binding pocket. ${ }^{272,306}$ The binding pockets of SAM and Cap are separated by a four amino-acid long stretch (residues 131-134). Ligand-binding sites with unclear functions have also been found on the surface of Nsp16. For example, a positively charged pocket close to the Cap binding pocket, possibly used to bind longer RNA, has been identified; ${ }^{307,309}$ on the back of the conservative catalytic center (Lys46-Asp130Lys170-Glu203), ${ }^{310,311}$ there is another ligand-binding pocket that accommodates adenosine and other small molecules with a heterocyclic ring. ${ }^{272,307}$ The interface of Nsp16-Nsp10, composed of multiple hydrophobic interactions and hydrogen bonds, largely overlaps with the interface of Nsp14-Nsp10. ${ }^{308}$ A key difference between the two interfaces lies in the a1-helix of Nsp10. Compared with the apo structure of Nsp10, the Nsp10 a1-helix retains the same conformation in the Nsp14-Nsp10 complex, whereas there is an obvious wiggle $\left(\sim 130^{\circ}\right)$ in the Nsp16 and Nsp10 complex. ${ }^{270}$

The Nsp16/Nsp10 complex in SARS-CoV-2 has high homology with those in other coronaviruses, and most residues that participate in catalysis and Cap/SAM binding are conserved. ${ }^{307,312}$ Therefore, inhibitors that target this protein are promising candidates for development into broad-spectrum antiviral agents. Inhibitor design for Nsp16 can be viewed from multiple perspectives. SAM analogs (such as sinefungin [SFG], a paninhibitor of MTase) can be utilized to occupy the SAM-binding pocket of Nsp16, inhibiting the activity of 2'-O-methyltransferase. $^{308}$ In the Nsp16-Nsp10-SFG complex (Fig. 7g), SFG has the same interactions with residues of Nsp16 as SAM does. ${ }^{308}$ Another approach is related to the protein conformation of Nsp16, which varies significantly before and after Cap is bound. If small molecules that stabilize the protein conformation can be identified, it may effectively inhibit the methylation of viral mRNA. $^{308}$ The specific ligand-binding pocket with unknown function in Nsp16 is also a potential target for inhibitor binding. ${ }^{307}$ Inhibitors that disrupt the interaction between Nsp16 and Nsp10 can also be applied, such as the peptide inhibitor mentioned above. $^{226,227}$ Finally, the construction of a live attenuated virus vaccine with Nsp16-defective SARS-CoV-2 may be possible. ${ }^{286}$

\section{ACCESSORY PROTEINS OF SARS-COV-2}

Accessory protein genes are interspaced between or within the viral structural protein genes, and have some genus or species specificity. SARS-CoV-2 encodes nine accessory proteins, including ORF3a (275 aa), ORF3b (22 aa), ORF6 (61 aa), ORF7a (121 aa), ORF7b (43 aa), ORF8 (121 aa), ORF9b (97 aa), ORF9c, and ORF10 (38 aa). ${ }^{313}$ These accessory proteins play essential roles in virushost interactions, ${ }^{314}$ affecting host innate immunity, autophagy, and apoptosis, as well as viral proliferation and virulence. ${ }^{315-317}$ Therefore, targeting these accessory proteins to interfere with
virus-host interactions is also a novel approach for treating COVID19. The structure of the four accessory proteins (ORF3a, ORF7a, ORF8, and ORF9b) that have been reported so far are described below.

\section{ORF3a protein}

ORF3a is highly conserved between SARS-CoV-2 and SARS-CoV $\left(72.7 \%\right.$ sequence identity), ${ }^{318}$ and is closely related to viral pathogenicity by disrupting the cellular physiology of the host cell. ${ }^{319,320}$ It not only promotes cytokine storms by activating NF$\mathrm{KB}$ signaling and NLRP3 inflammasomes, ${ }^{321}$ but also regulates host 
a

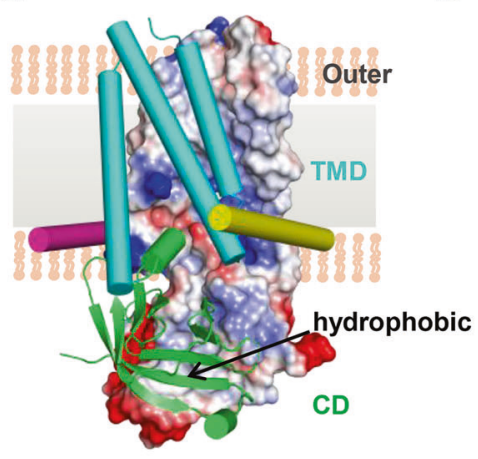

ORF3a

(PDB ID: 7KJR) b

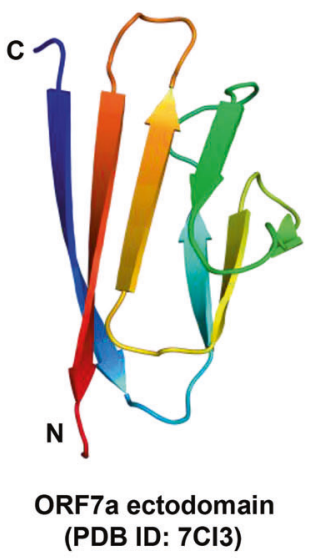

C

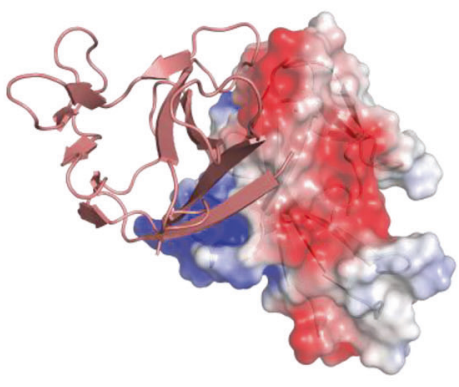

ORF8

(PDB ID: 7JX6) d

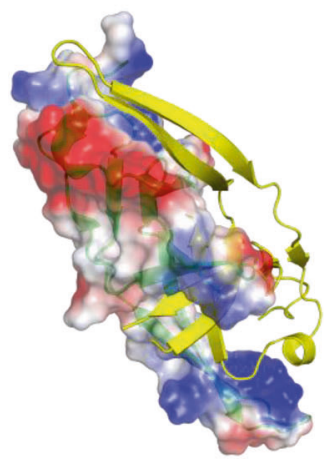

ORF9b

(PDB ID: 6Z4U) e

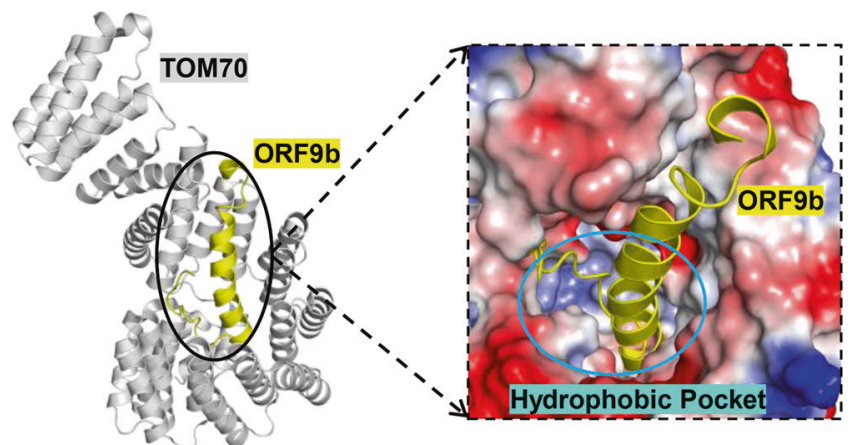

ORF9b-TOM70

(PDB ID: 7KDT)

Fig. 8 Structures of accessory proteins. a-d Overall structure of ORF3a, ORF7a, ORF8, and ORF 9b, respectively. e Structure of ORF9b in complex with human protein TOM70, a subunit of the mitochondrial import receptor. ORF9b is shown as yellow cartoon. TOM70 is illustrated with electrostatic surface on the right. The ORF9b binds to the hydrophobic pocket of TOM70 and occupies its binding site

cell apoptosis and autophagy. ${ }^{322,323}$ Furthermore, ORF3a is another viroporin in addition to the envelope (E) protein, ${ }^{324}$ which participates in the release of viral particles as an ion channel. $^{325}$

ORF3a is the largest accessory protein in SARS-CoV-2. ${ }^{319}$ The crystal structure is an oligomer including a dimer and tetramer (PDB ID 7KJR), and the tetramer conformation is formed by two dimers $^{326}$ (Fig. 8a). A pair of short a-helices divide the dimer structure into the transmembrane domain (TMD) that forms a central channel and the cytosolic domain (CD) located in the cytosol. The TM domain consists of three transmembrane $a-$ helices and the $C D$ domain forms a $\beta$-barrel comprising eight antiparallel $\beta$-strands. The stability of the dimer is dependent on multiple non-covalent interactions ( $\pi-\pi$ stacking and hydrophobic interactions) between two CD domains. In addition, several functional domains are also present in ORF3a, such as a cysteine-rich pocket that facilitates oligomerization and a tyrosine-based sorting motif $(\mathrm{YXX} \varphi)$ that mediates intracellular and extracellular transport. ${ }^{313,326-328}$

ORF3a binds to host heme oxygenase HMOX $1,{ }^{329}$ which plays an essential role in anti-inflammatory effects via the NLRPS pathway. ${ }^{330-332}$ Treatment with compounds that inhibit the interaction between ORF3a and HMOX1 is an effective strategy for the treatment of COVID-19. Additionally, anti-ORF3a antibodies have been found in the plasma of convalescent COVID-19 patients. $^{333,334}$
ORF7a protein

SARS-CoV-2 ORF7a is a type-I transmembrane protein composed of an N-terminal signal peptide (residues 1-15), Ig-like ectodomain (residues 16-96), transmembrane region (residues 97-116), and ER retention motif (KRKTE) (residues 117-121). ${ }^{335}$ The structure of the Ig-like ectodomain is similar to the intracellular adhesion molecule-2 (ICAM-2) of the immunoglobulin superfamily, ${ }^{6,336}$ which is folded into a $\beta$-sandwich by seven $\beta$-strands (Fig. 8b); two pairs of intramolecular disulfide bonds (Cys23-Cys58 and Cys35-Cys67) participate in the stability of the structure. ${ }^{335}$

ORF7a triggers an immune response in host cells. ${ }^{337}$ It not only activates NF-KB signaling and induces the expression of proinflammatory cytokines and chemokines ${ }^{338}$ but also inhibits the induction of type-I interferon by blocking STAT2 phosphorylation. ${ }^{339-341}$ Therefore, ORF7a is an attractive therapeutic target for COVID-19. Notably, the C-terminus of ORF7a plays a nonnegligible role in host immunomodulation, but this region has a higher mutation frequency than the Ig-like ectodomain. This variability would be important to the design of broad-spectrum antiviral agents against this protein.

\section{ORF8 protein}

As the most variable accessory protein in $\beta$-coronaviruses, ORF8 frequently undergoes gene recombination, deletion, and substitution, which is associated with pathogenicity and adaptive evolution. $^{342-344}$ SARS-CoV-2 ORF8 is a multifunctional protein 
that induces host cell apoptosis, ${ }^{345}$ suppresses the host innate immune response by downregulating the class I major histocompatibility complex (MHC-I)-mediated viral antigen presentation, ${ }^{346}$ and functions as an antagonist of type-I interferon (IFN). ${ }^{131,339,347,348}$ Therefore, ORF8 is considered a potential antiviral target.

SARS-CoV-2 ORF8 consists of 121 amino acids, including the $\mathrm{N}$-terminal TM domain (residues 1-17) and the central lg-like domain (residues 18-121). The TM domain is responsible for endoplasmic reticulum (ER) import and secretion, and the lg-like domain can interact with multiple host factors involved in pulmonary inflammation and fibrogenesis. ${ }^{349,350}$ The crystal structure of SARS-CoV-2 ORF8 is a homodimer (PDB ID 7JTL), and each monomer comprises eight antiparallel $\beta$-strands with three pairs of intramolecular disulfide bridges between the strands $^{351}$ (Fig. 8c). Drugs targeting ORF8 can be designed based on the structure to disrupt interactions between ORF8 and multiple host proteins, such as lysyl oxidase (LOX), interleukin 17 receptor (IL17RA), and growth/differentiation factor 15 (GDF15). ${ }^{349}$ Additionally, ORF8 protein elicits robust antibody responses in the host, and these antibodies have become the major serological marker of SARS-CoV-2 infection. ${ }^{329,337,352}$ In the future, these specific antibodies can be screened and identified.

\section{ORF9b}

ORF9b is an accessory protein unique to SARS-CoV and SARS-CoV$2 .{ }^{353}$ Both SARS-CoV ORF9b and SARS-CoV-2 ORF9b proteins are dimerized and assembled into a tent-like shape by two intertwined monomers (PDB ID 2CME and 6Z4U) ${ }^{354}$ (Fig. 8d). The monomer consists entirely of $\beta$-strands. A number of charged residues are distributed on the surface and a hydrophobic central tunnel for lipid binding is located inside the dimer. ${ }^{354}$

SARS-CoV-2 ORF9b specifically binds to the mitochondrial surface receptor protein TOM70, ${ }^{350}$ which cooperates with molecular chaperone Hsp90 to promote the transfer of preproteins to mitochondria and activates the host antiviral immune response. ${ }^{355,356}$ The $\mathrm{N}$-terminal tetratricopeptide repeats (TPRs) of TOM70 are associated with the Hsp90 C-terminal EEVD motif, and the C-terminal TPRs bind to the mitochondrial preprotein. In the ORF9b-TOM70 complex (PDB ID 7KDT and 7DHG) (Fig. 8e), a single ORF9b binds to the hydrophobic pocket at the C-terminal domain (CTD) of Tom70, occupying the preprotein binding site and allosterically inhibiting the interaction between Tom70 and Hsp90. ${ }^{350,353}$ This ultimately blocks host mitophagy and interferon signaling. ${ }^{350,357}$ Therefore, inhibitors that disrupt the interaction between ORF9b and Tom70 could be screened to interfere with viral proliferation. Antibodies targeted to ORF9b have also been observed in the plasma of convalescent COVID-19 patients. ${ }^{358}$

\section{CONCLUSION}

Despite great progress in the development of antiviral drugs based on the SARS-CoV-2 protein structures, there are still some challenges in this field. Firstly, although most protein structures have been determined, the functions of SARS-CoV-2 proteins have not been fully characterized. For example, recent studies surprisingly found that Nsp12 not only employ NDP distinguishing from conventional NTP as a substrate to synthesis RNA, but also likely display both exoribonuclease and proofreading activity, and its proofreading activity increased with the combination of Nsp14ExoN. ${ }^{359}$ Secondly, the molecular basis of dynamic interactions of SARS-CoV-2 with host need to be further investigated. For example, a large number of host proteins that interact with SARS-CoV-2 proteins have been identified, but the information of the complex structures of the viral proteins of SARS-CoV-2 with host proteins is still limited. Lastly, these drugs targeting a single site are particularly vulnerable to the evolution of drug resistance through random viral mutation. The therapeutic effects of structural-based drugs on the numerous emerging SARS-CoV-2 variants need to be further analyzed. Simultaneously it might be a better choice to develop drugs targeting multiple key sites or conserved binding sites of viral protein with host proteins in the life cycle of SARS-CoV-2. Currently, we have been hunted ways to abolish the virus after the variants happened, however, the most important question is how to take precautions before the pandemic variants.

In conclusion, we summary the representative protein structures of SARS-CoV-2 and the structural-based drug design utilizing these proteins and predict the structures of those proteins that lack precise structural information, including $M, N s p 4$ and Nsp6 by using the latest prediction tool, like Alphafold 2. The review is aimed at providing theoretical insight for mitigating the current COVID-19 pandemic and potential future coronavirus outbreaks.

\section{ACKNOWLEDGEMENTS}

This work was funded by a grant to W.C. from the National Natural Science Foundation of China (Grant No. 32071236 and 31870836), the 1.3.5 Project for Disciplines Excellence of West China Hospital, Sichuan University (ZYYC20005), and Key Science and Technology Research Projects in Key Areas of the Corps (Grant No. 2018AB019).

\section{AUTHOR CONTRIBUTIONS}

W.C. conceived the manuscript. W.Y. wrote the initial draft of the manuscript. Y.Z. drew figures. X.Z. participated in the revision of manuscript. All authors have read and approved the article. W.C. had final approval of the final manuscript.

\section{ADDITIONAL INFORMATION}

Competing interests: The authors declare no competing interests.

\section{REFERENCES}

1. Jin, Y. F. et al. Virology, epidemiology, pathogenesis, and control of COVID-19. Viruses 12, 372 (2020).

2. Mittal, A. et al. COVID-19 pandemic: insights into structure, function, and hACE2 receptor recognition by SARS-CoV-2. PLoS Pathog. 16, e1008762 (2020).

3. Chan, J. F. et al. A familial cluster of pneumonia associated with the 2019 novel coronavirus indicating person-to-person transmission: a study of a family cluster. Lancet 395, 514-523 (2020).

4. Lu, R. J. et al. Genomic characterisation and epidemiology of 2019 novel coronavirus: implications for virus origins and receptor binding. Lancet 395, 565-574 (2020).

5. Wrobel, A. et al. SARS-CoV-2 and bat RaTG13 spike glycoprotein structures inform on virus evolution and furin-cleavage effects. Nat. Struct. Mol. Biol. 27, 763-767 (2020).

6. Arya, R. et al. Structural insights into SARS-CoV-2 proteins. J. Mol. Biol. 433, 166725 (2020).

7. Zumla, A., Chan, J., Azhar, E., Hui, D. \& Yuen, K. Coronaviruses-drug discovery and therapeutic options. Nat. Rev. Drug Discov. 15, 327-347 (2016).

8. Helmy, Y. et al. The COVID-19 pandemic: a comprehensive review of taxonomy, genetics, epidemiology, diagnosis, treatment, and control. J. Clin. Med. 9, 1225 (2020).

9. Posthuma, C., Te Velthuis, A. \& Snijder, E. Nidovirus RNA polymerases: complex enzymes handling exceptional RNA genomes. Virus Res. 234, 58-73 (2017).

10. $\mathrm{Wu}, \mathrm{F}$. et al. A new coronavirus associated with human respiratory disease in China. Nature 579, 265-269 (2020).

11. Harrison, A., Lin, T. \& Wang, P. Mechanisms of SARS-CoV-2 transmission and pathogenesis. Trends Immunol. 41, 1100-1115 (2020).

12. Michel, C., Mayer, C., Poch, O. \& Thompson, J. Characterization of accessory genes in coronavirus genomes. Virol. J. 17, 131 (2020).

13. Liu, C. et al. Research and development on therapeutic agents and vaccines for COVID-19 and related human coronavirus diseases. ACS Cent. Sci. 6, 315-331 (2020).

14. Su, S., Du, L. Y. \& Jiang, S. B. Learning from the past: development of safe and effective COVID-19 vaccines. Nat. Rev. Microbiol. 19, 211-219 (2021).

15. Yang, J. Y. et al. A vaccine targeting the RBD of the S protein of SARS-CoV-2 induces protective immunity. Nature 586, 572-577 (2020).

16. Krammer, F. SARS-CoV-2 vaccines in development. Nature 586, 516-527 (2020). 
17. Hilligan, K. L. et al. Intravenous administration of BCG protects mice against lethal SARS-CoV-2 challenge. J. Exp. Med. 219, e20211862 (2022).

18. Dube, T., Ghosh, A., Mishra, J., Kompella, U. B. \& Panda, J. J. Repurposed drugs, molecular vaccines, immune-modulators, and nanotherapeutics to treat and prevent COVID-19 associated with SARS-CoV-2, a deadly nanovector. Adv. Ther. 4, 2000172 (2021)

19. Lu, L., Su, S., Yang, H. T. \& Jiang, S. B. Antivirals with common targets against highly pathogenic viruses. Cell 184, 1604-1620 (2021).

20. Runfeng, L. et al. Lianhuaqingwen exerts anti-viral and anti-inflammatory activity against novel coronavirus (SARS-CoV-2). Pharmacol. Res 156, 104761 (2020).

21. Samuel, C. E. Antiviral actions of interferons. Clin. Microbiol. Rev. 14, 778-809 (2001).

22. Russell, C. D., Millar, J. E. \& Baillie, J. K. Clinical evidence does not support corticosteroid treatment for 2019-nCoV lung injury. Lancet 395, 473-475 (2020).

23. Sharun, K. et al. Antibody-based immunotherapeutics and use of convalescent plasma to counter COVID-19: advances and prospects. Expert. Opin. Biol. Ther. 20, 1033-1046 (2020).

24. Golchin, A., Seyedjafari, E. \& Ardeshirylajimi, A. Mesenchymal stem cell therapy for COVID-19: present or future. Stem Cell Rev. Rep. 16, 427-433 (2020).

25. Wang, R., Chen, J. \& Wei, G. W. Mechanisms of SARS-CoV-2 evolution revealing vaccine-resistant mutations in Europe and America. J. Phys. Chem. Lett. 12 11850-11857 (2021).

26. Eberle, R. et al. The repurposed drugs suramin and quinacrine cooperatively inhibit SARS-CoV-2 3CL ${ }^{\text {pro }}$ in vitro. Viruses 13, 873 (2021).

27. Tortorici, M. \& Veesler, D. Structural insights into coronavirus entry. Adv. Virus Res. 105, 93-116 (2019).

28. Walls, A. et al. Structure, function, and antigenicity of the SARS-CoV-2 spike glycoprotein. Cell 181, 281-292 (2020).

29. Zhang, J., Xiao, T. S., Cai, Y. F. \& Chen, B. Structure of SARS-CoV-2 spike protein. Curr. Opin. Virol. 50, 173-182 (2021).

30. Lan, J. et al. Structure of the SARS-CoV-2 spike receptor-binding domain bound to the ACE2 receptor. Nature 581, 215-220 (2020).

31. Cai, Y. F. et al. Distinct conformational states of SARS-CoV-2 spike protein. Science 369, 1586-1592 (2020).

32. Liu, C. et al. The architecture of inactivated SARS-CoV-2 with postfusion Spikes revealed by Cryo-EM and Cryo-ET. Structure 28, 1218-1224 (2020).

33. Jackson, C. B., Farzan, M., Chen, B. \& Choe, H. Mechanisms of SARS-CoV-2 entry into cells. Nat. Rev. Mol. Cell Biol. 23, 3-20 (2022).

34. Yao, $\mathrm{H}$. et al. Molecular architecture of the SARS-CoV-2 virus. Cell 183, 730-738 (2020).

35. Xia, S. et al. Fusion mechanism of 2019-nCoV and fusion inhibitors targeting HR1 domain in spike protein. Cell Mol. Immunol. 17, 765-767 (2020).

36. Caly, L., Druce, J. D., Catton, M. G., Jans, D. A. \& Wagstaff, K. M. The FDA-approved drug ivermectin inhibits the replication of SARS-CoV-2 in vitro. Antivir. Res 178, 104787 (2020)

37. de Oliveira, O., Rocha, G., Paluch, A. \& Costa, L. Repurposing approved drugs as inhibitors of SARS-CoV-2 S-protein from molecular modeling and virtual screening. J. Biomol. Struct. Dyn. 39, 3924-3933 (2021).

38. Vankadari, N. Arbidol: a potential antiviral drug for the treatment of SARS-CoV-2 by blocking trimerization of the spike glycoprotein. Int. J. Antimicrob. Agents 56, 105998 (2020)

39. Padhi, A., Seal, A., Khan, J., Ahamed, M. \& Tripathi, T. Unraveling the mechanism of arbidol binding and inhibition of SARS-CoV-2: insights from atomistic simulations. Eur. J. Pharmacol. 894, 173836 (2021).

40. Wang, $X$. et al. The anti-influenza virus drug, arbidol is an efficient inhibitor of SARS-CoV-2 in vitro. Cell Discov. 6, 28 (2020).

41. Xia, S. et al. Inhibition of SARS-CoV-2 (previously 2019-nCoV) infection by a highly potent pan-coronavirus fusion inhibitor targeting its spike protein that harbors a high capacity to mediate membrane fusion. Cell Res. 30, 343-355 (2020).

42. Hastie, K. M. et al. Defining variant-resistant epitopes targeted by SARS-CoV-2 antibodies: a global consortium study. Science 374, 472-478 (2021).

43. Chi, X. Y. et al. A neutralizing human antibody binds to the $\mathrm{N}$-terminal domain of the spike protein of SARS-CoV-2. Science 369, 650-655 (2020).

44. Wang, N. et al. Structure-based development of human antibody cocktails against SARS-CoV-2. Cell Res. 31, 101-103 (2021).

45. Voss, W. et al. Prevalent, protective, and convergent lgG recognition of SARSCoV-2 non-RBD spike epitopes. Science 372, 1108-1112 (2021).

46. McCallum, M. et al. N-terminal domain antigenic mapping reveals a site of vulnerability for SARS-CoV-2. Cell 184, 2332-2347 (2021).

47. Yuan, $M$. et al. A highly conserved cryptic epitope in the receptor binding domains of SARS-CoV-2 and SARS-CoV. Science 368, 630-633 (2020),

48. Song, G. et al. Cross-reactive serum and memory B-cell responses to spike protein in SARS-CoV-2 and endemic coronavirus infection. Nat. Commun. 12, 2938 (2021).
49. Zheng, Z. Q. et al. Monoclonal antibodies for the S2 subunit of spike of SARSCoV-1 cross-react with the newly-emerged SARS-CoV-2. Eur. Surveill. 25, 2000291 (2020).

50. Pinto, D. et al. Cross-neutralization of SARS-CoV-2 by a human monoclonal SARS-CoV antibody. Nature 583, 290-295 (2020).

51. Walls, A. et al. Glycan shield and epitope masking of a coronavirus spike protein observed by cryo-electron microscopy. Nat. Struct. Mol. Biol. 23, 899-905 (2016).

52. Watanabe, Y., Allen, J., Wrapp, D., McLellan, J. \& Crispin, M. Site-specific glycan analysis of the SARS-CoV-2 spike. Science 369, 330-333 (2020).

53. McCallum, M., Walls, A., Bowen, J., Corti, D. \& Veesler, D. Structure-guided covalent stabilization of coronavirus spike glycoprotein trimers in the closed conformation. Nat. Struct. Mol. Biol. 27, 942-949 (2020).

54. Juraszek, J. et al. Stabilizing the closed SARS-CoV-2 spike trimer. Nat. Commun. 12, 244 (2021).

55. Shajahan, A., Pepi, L., Rouhani, D., Heiss, C. \& Azadi, P. Glycosylation of SARS CoV-2: structural and functional insights. Anal. Bioanal. Chem. 413, 7179-7193 (2021).

56. Papageorgiou, A. C. \& Mohsin, I. The SARS-CoV-2 Spike glycoprotein as a drug and vaccine target: structural insights into its complexes with ACE2 and antibodies. Cells 9, 2343 (2020).

57. Shajahan, A., Supekar, N. T., Gleinich, A. S. \& Azadi, P. Deducing the N- and O-glycosylation profile of the spike protein of novel coronavirus SARS-CoV-2. Glycobiology 30, 981-988 (2020).

58. Hamming, I. et al. Tissue distribution of ACE2 protein, the functional receptor for SARS coronavirus. a first step in understanding SARS pathogenesis. J. Pathol. 203, 631-637 (2004).

59. Yan, R. H. et al. Structural basis for the recognition of SARS-CoV-2 by full-length human ACE2. Science 367, 1444-1448 (2020).

60. Pedersen, K., Sriramula, S., Chhabra, K., Xia, H. \& Lazartigues, E. Species-specific inhibitor sensitivity of angiotensin-converting enzyme 2 (ACE2) and its implication for ACE2 activity assays. Am. J. Physiol. Regul. Integr. Comp. Physiol. 301, 1293-1299 (2011)

61. Diz, D. et al. Injections of angiotensin-converting enzyme 2 inhibitor MLN4760 into nucleus tractus solitarii reduce baroreceptor reflex sensitivity for heart rate control in rats. Exp. Physiol. 93, 694-700 (2008).

62. Huentelman, M. et al. Structure-based discovery of a novel angiotensinconverting enzyme 2 inhibitor. Hypertension 44, 903-906 (2004).

63. Bollavaram, K. et al. Multiple sites on SARS-CoV-2 spike protein are susceptible to proteolysis by cathepsins B, K, L, S, and V. Protein Sci. 30, 1131-1143 (2021).

64. Hoffmann, M. et al. SARS-CoV-2 cell entry depends on ACE2 and TMPRSS2 and is blocked by a clinically proven protease inhibitor. Cell 181, 271-280 (2020).

65. Belouzard, S., Chu, V. \& Whittaker, G. Activation of the SARS coronavirus spike protein via sequential proteolytic cleavage at two distinct sites. Proc. Natl Acad. Sci. USA 106, 5871-5876 (2009).

66. Millet, J. \& Whittaker, G. Host cell proteases: critical determinants of coronavirus tropism and pathogenesis. Virus Res. 202, 120-134 (2015)

67. Cheng, Y. et al. Furin inhibitors block SARS-CoV-2 Spike protein cleavage to suppress virus production and cytopathic effects. Cell Rep. 33, 108254 (2020).

68. Glinsky, G. Tripartite combination of candidate pandemic mitigation agents: vitamin $d$, quercetin, and estradiol manifest properties of medicinal agents for targeted mitigation of the COVID-19 pandemic defined by genomics-guided tracing of SARS-CoV-2 targets in human cells. Biomedicines 8, 129 (2020).

69. Palmeira, A. et al. Preliminary virtual screening studies to identify GRP78 inhibitors which may interfere with SARS-CoV-2 infection. Pharmaceuticals 13, 132 (2020).

70. Fenizia, C. et al. SARS-CoV-2 entry: at the crossroads of CD147 and ACE2. Cells 10, 1434 (2021)

71. Starr, T. N. et al. Deep mutational scanning of SARS-CoV-2 receptor binding domain reveals constraints on folding and ACE2 binding. Cell 182, 1295-1310 (2020).

72. Korber, B. et al. Tracking changes in SARS-CoV-2 Spike: evidence that D614G increases infectivity of the COVID-19 virus. Cell 182, 812-827 (2020).

73. Hoffmann, M., Kleine-Weber, H. \& Pöhlmann, S. A multibasic cleavage site in the Spike protein of SARS-CoV-2 is essential for infection of human lung cells. Mol. Cell 78, 779-784 (2020).

74. McCarthy, K. R. et al. Recurrent deletions in the SARS-CoV-2 spike glycoprotein drive antibody escape. Science 371, 1139-1142 (2021).

75. Gomez, C. E., Perdiguero, B. \& Esteban, M. Emerging SARS-CoV-2 variants and impact in global vaccination programs against SARS-CoV-2/COVID-19. Vaccines 9, 243 (2021).

76. Wang, Z. J. et al. mRNA vaccine-elicited antibodies to SARS-CoV-2 and circulating variants. Nature 592, 616-622 (2021).

77. Lazarevic, I., Pravica, V., Miljanovic, D. \& Cupic, M. Immune evasion of SARS-CoV2 emerging variants: what have we learnt so far? Viruses 13, 1192 (2021).

78. Deng, X. D. et al. Transmission, infectivity, and neutralization of a spike L452R SARS-CoV-2 variant. Cell 184, 3426-3437 (2021). 
79. Liu, Z. M. et al. Identification of SARS-CoV-2 spike mutations that attenuate monoclonal and serum antibody neutralization. Cell Host Microbe 29, 477-488 (2021).

80. Miller, N. L., Clark, T., Raman, R. \& Sasisekharan, R. Insights on the mutational landscape of the SARS-CoV-2 Omicron variant. bioRxiv https://doi.org/10.1101/ 2021.12.06.471499 (2021).

81. Saxena, S. K. et al. Characterization of the novel SARS-CoV-2 Omicron (B.1.1.529) Variant of Concern and its global perspective. J. Med. Virol. https://doi.org/ 10.1002/jmv.27524 (2021)

82. He, X. M., Hong, W. Q., Pan, X. Y., Lu, G. W. \& Wei, X. W. SARS-CoV-2 Omicron variant: characteristics and prevention. MedComm 16, 838-845 (2020).

83. Gu, H. et al. Probable transmission of SARS-CoV-2 Omicron Variant in quarantine hotel, Hong Kong, China, November 2021. Emerg. Infect. Dis. https://doi.org/ 10.3201/eid2802.212422 (2021).

84. Lu, X., Pan, J. A., Tao, J. \& Guo, D. SARS-CoV nucleocapsid protein antagonizes IFN- $\beta$ response by targeting initial step of IFN- $\beta$ induction pathway, and its C-terminal region is critical for the antagonism. Virus Genes 42, 37-45 (2011).

85. Bai, Z. H., Cao, Y., Liu, W. J. \& Li, J. The SARS-CoV-2 nucleocapsid protein and its role in viral structure, biological functions, and a potential target for drug or vaccine mitigation. Viruses 13, 1115 (2021).

86. Kannan, S., Subbaram, K., Ali, S. \& Kannan, H. Molecular characterization and amino acid homology of nucleocapsid (N) protein in SARS-CoV-1, SARS-CoV-2, MERS-CoV, and Bat coronavirus. J. Pure Appl. Microbiol. 14, 757-763 (2020).

87. Wootton, S. K., Rowland, R. R. \& Yoo, D. Phosphorylation of the porcine reproductive and respiratory syndrome virus nucleocapsid protein. J. Virol. 76, 10569-10576 (2002).

88. Lin, S. Y. et al. Structural basis for the identification of the N-terminal domain of coronavirus nucleocapsid protein as an antiviral target. J. Med. Chem. 57, 2247-2257 (2014).

89. Guo, Y. et al. Crystal structure of the core region of Hantavirus nucleocapsid protein reveals the mechanism for ribonucleoprotein complex formation. J. Virol. 90, 1048-1061 (2016).

90. Dinesh, D. C. et al. Structural basis of RNA recognition by the SARS-CoV-2 nucleocapsid phosphoprotein. PLoS Pathog. 16, e1009100 (2020).

91. He, Y. X. et al. Mapping of antigenic sites on the nucleocapsid protein of the severe acute respiratory syndrome coronavirus. J. Clin. Microbiol. 42, 5309-5314 (2004).

92. Kang, S. et al. A SARS-CoV-2 antibody curbs viral nucleocapsid protein-induced complement hyperactivation. Nat. Commun. 12, 2697 (2021).

93. Matsuo, T. Viewing SARS-CoV-2 nucleocapsid protein in terms of molecular flexibility. Biology 10, 454 (2021).

94. Peng, Y. et al. Structures of the SARS-CoV-2 nucleocapsid and their perspectives for drug design. EMBO J. 39, e105938 (2020).

95. Verdiá-Báguena, C. et al. Coronavirus E protein forms ion channels with functionally and structurally-involved membrane lipids. Virology 432, 485-494 (2012).

96. Schoeman, D. \& Fielding, B. Coronavirus envelope protein: current knowledge. Virol. J. 16, 69 (2019).

97. Nieto-Torres, J. L. et al. Severe acute respiratory syndrome coronavirus E protein transports calcium ions and activates the NLRP3 inflammasome. Virology 485, 330-339 (2015).

98. Mandala, V. et al. Structure and drug binding of the SARS-CoV-2 envelope protein transmembrane domain in lipid bilayers. Nat. Struct. Mol. Biol. 27, 1202-1208 (2020).

99. Pervushin, K. et al. Structure and inhibition of the SARS coronavirus envelope protein ion channel. PLoS Pathog. 5, e1000511 (2009).

100. Li, Y. et al. Structure of Crumbs tail in complex with the PALS1 PDZ-SH3-GK tandem reveals a highly specific assembly mechanism for the apical Crumbs complex. Proc. Natl Acad. Sci. USA 111, 17444-17449 (2014).

101. Roh, M. et al. The Maguk protein, Pals1, functions as an adapter, linking mammalian homologues of Crumbs and Discs Lost. J. Cell Biol. 157, 161-172 (2002).

102. Wells, C. et al. A Rich1/Amot complex regulates the Cdc42 GTPase and apicalpolarity proteins in epithelial cells. Cell 125, 535-548 (2006).

103. Chai, J. et al. Structural basis for SARS-CoV-2 envelope protein recognition of human cell junction protein PALS1. Nat. Commun. 12, 3433 (2021).

104. Christensen, N. et al. PDZ domains as drug targets. Adv. Ther. 2, 1800143 (2019).

105. Javorsky, A., Humbert, P. O. \& Kvansakul, M. Structural basis of coronavirus E protein interactions with human PALS1 PDZ domain. Commun. Biol. 4, 724 (2021).

106. O'Neill, L. \& Netea, M. BCG-induced trained immunity: can it offer protection against COVID-19? Nat. Rev. Immunol. 20, 335-337 (2020).

107. Nuovo, G. et al. Strong homology between SARS-CoV-2 envelope protein and a Mycobacterium sp. antigen allows rapid diagnosis of Mycobacterial infections and may provide specific anti-SARS-CoV-2 immunity via the BCG vaccine. Ann. Diagn. Pathol. 48, 151600 (2020).
108. Koeken, V. Controlling inflammation in the elderly with BCG vaccination. Sci. Adv. 7, eabk1668 (2021).

109. de Haan, C. A. M., Smeets, M., Vernooij, F., Vennema, H. \& Rottier, P. J. M. Mapping of the coronavirus membrane protein domains involved in interaction with the spike protein. J. Virol. 73, 7441-7452 (1999).

110. Neuman, B. et al. A structural analysis of $M$ protein in coronavirus assembly and morphology. J. Struct. Biol. 174, 11-22 (2011).

111. Li, J. J. et al. Virus-host interactome and proteomic survey reveal potential virulence factors influencing SARS-CoV-2 pathogenesis. Med 2, 99-112 (2021).

112. Astuti, I. \& Ysrafil Severe acute respiratory syndrome coronavirus 2 (SARS-CoV-2): an overview of viral structure and host response. Diabetes Metab. Syndr. 14, 407-412 (2020).

113. Wong, N. \& Saier, M. The SARS-coronavirus infection cycle: a survey of viral membrane proteins, their functional interactions and pathogenesis. Int. J. Mol. Sci. 22, 1308 (2021).

114. Mahtarin, R. et al. Structure and dynamics of membrane protein in SARS-CoV-2. J. Biomol. Struct. Dyn. https://doi.org/10.1080/07391102.2020.1861983 (2020).

115. Thomas, S. The structure of the membrane protein of SARS-CoV-2 resembles the sugar transporter SemiSWEET. Pathog. Immun. 5, 342-363 (2020).

116. Perrier, A. et al. The C-terminal domain of the MERS coronavirus $M$ protein contains a trans-Golgi network localization signal. J. Biol. Chem. 294, 14406-14421 (2019).

117. Hemmati, S., Behzadipour, Y. \& Haddad, M. Decoding the proteome of severe acute respiratory syndrome coronavirus 2 (SARS-CoV-2) for cell-penetrating peptides involved in pathogenesis or applicable as drug delivery vectors. Infect. Genet. Evol. 85, 104474 (2020).

118. Tseng, Y., Chang, C., Wang, S., Huang, K. \& Wang, C. Identifying SARS-CoV membrane protein amino acid residues linked to virus-like particle assembly. PLoS ONE 8, e64013 (2013).

119. Pang, $\mathrm{H}$. et al. Protective humoral responses to severe acute respiratory syndrome-associated coronavirus: implications for the design of an effective protein-based vaccine. J. Gen. Virol. 85, 3109-3113 (2004).

120. Liu, J. et al. The membrane protein of severe acute respiratory syndrome coronavirus acts as a dominant immunogen revealed by a clustering region of novel functionally and structurally defined cytotoxic T-lymphocyte epitopes. J. Infect. Dis. 202, 1171-1180 (2010).

121. Khan, F., Kang, T., Ali, H. \& Lai, D. Remdesivir strongly binds to RNA-dependent RNA polymerase, membrane protein, and main protease of SARS-CoV-2: indication from molecular modeling and simulations. Front. Pharmacol. 12, 710778 (2021).

122. Hagemeijer, M. et al. Membrane rearrangements mediated by coronavirus nonstructural proteins 3 and 4. Virology 458, 125-135 (2014).

123. Angelini, M. M., Akhlaghpour, M., Neuman, B. W. \& Buchmeier, M. J. Severe acute respiratory syndrome coronavirus nonstructural proteins 3, 4, and 6 induce double-membrane vesicles. mBio 4, e00524-13 (2013).

124. Thomas, S. Mapping the nonstructural transmembrane proteins of severe acute respiratory syndrome coronavirus 2. J. Comput. Biol. 28, 909-921 (2021).

125. Rogstam, A. et al. Crystal structure of non-structural protein 10 from severe acute respiratory syndrome coronavirus-2. Int. J. Mol. Sci. 21, 7375 (2020).

126. Yuan, S. et al. Nonstructural protein 1 of SARS-CoV-2 is a potent pathogenicity factor redirecting host protein synthesis machinery toward viral RNA. Mol. Cell 80, 1055-1066 (2020).

127. Huang, C. et al. SARS coronavirus nsp1 protein induces template-dependent endonucleolytic cleavage of mRNAs: viral mRNAs are resistant to nsp1-induced RNA cleavage. PLoS Pathog. 7, e1002433 (2011)

128. Kamitani, W. et al. Severe acute respiratory syndrome coronavirus nsp1 protein suppresses host gene expression by promoting host mRNA degradation. Proc. Natl Acad. Sci. USA 103, 12885-12890 (2006).

129. Lokugamage, K., Narayanan, K., Huang, C. \& Makino, S. Severe acute respiratory syndrome coronavirus protein $\mathrm{nsp} 1$ is a novel eukaryotic translation inhibitor that represses multiple steps of translation initiation. J. Virol. 86, 13598-13608 (2012).

130. Narayanan, K. et al. Severe acute respiratory syndrome coronavirus nsp1 suppresses host gene expression, including that of type I interferon, in infected cells. J. Virol. 82, 4471-4479 (2008).

131. Lei, X. B. et al. Activation and evasion of type I interferon responses by SARSCoV-2. Nat. Commun. 11, 3810 (2020).

132. Züst, R. et al. Coronavirus non-structural protein 1 is a major pathogenicity factor: implications for the rational design of coronavirus vaccines. PLoS Pathog. 3, e109 (2007).

133. Wathelet, M., Orr, M., Frieman, M. \& Baric, R. Severe acute respiratory syndrome coronavirus evades antiviral signaling: role of nsp1 and rational design of an attenuated strain. J. Virol. 81, 11620-11633 (2007).

134. Clark, L., Green, T. \& Petit, C. Structure of nonstructural protein 1 from SARS-CoV2. J. Virol. 95, e02019-e02020 (2021). 
135. Thoms, M. et al. Structural basis for translational shutdown and immune evasion by the Nsp1 protein of SARS-CoV-2. Science 369, 1249-1255 (2020).

136. Tidu, A. et al. The viral protein Nsp1 acts as a ribosome gatekeeper for shutting down host translation and fostering SARS-CoV-2 translation. RNA 27, 253-264 (2020).

137. Schubert, K. et al. SARS-CoV-2 Nsp1 binds the ribosomal mRNA channel to inhibit translation. Nat. Struct. Mol. Biol. 27, 959-966 (2020).

138. Zhao, K. et al. Structural basis and function of the $\mathrm{N}$ terminus of SARS-CoV-2 nonstructural protein 1. Microbiol. Spectr. 9, e0016921 (2021).

139. Vankadari, N., Jeyasankar, N. \& Lopes, W. Structure of the SARS-CoV-2 Nsp1/5'untranslated region complex and implications for potential therapeutic targets, a vaccine, and virulence. J. Phys. Chem. Lett. 11, 9659-9668 (2020).

140. Zheng, Y. X. et al. Nsp2 has the potential to be a drug target revealed by global identification of SARS-CoV-2 Nsp2-interacting proteins. Acta Biochim. Biophys. Sin. 53, 1134-1141 (2021).

141. Verba, K. et al. CryoEM and Al reveal a structure of SARS-CoV-2 Nsp2, a multifunctional protein involved in key host processes. Res. Sq. 3, 515215 (2021).

142. Tollenaere, M. et al. GIGYF1/2-driven cooperation between ZNF598 and TTP in posttranscriptional regulation of inflammatory signaling. Cell Rep. 26, 3511-3521 (2019)

143. Cornillez-Ty, C., Liao, L., Yates, J., Kuhn, P. \& Buchmeier, M. Severe acute respiratory syndrome coronavirus nonstructural protein 2 interacts with a host protein complex involved in mitochondrial biogenesis and intracellular signaling. J. Virol. 83, 10314-10318 (2009).

144. von Brunn, A. et al. Analysis of intraviral protein-protein interactions of the SARS coronavirus ORFeome. PLOS ONE 2, e459 (2007).

145. Ma, J., Chen, Y. Y., Wu, W. \& Chen, Z. Z. Structure and function of N-terminal Zinc finger domain of SARS-CoV-2 Nsp2. Virol. Sin. 36, 1104-1112 (2021).

146. Maiti, S., Banerjee, A., Nazmeen, A., Kanwar, M. \& Das, S. Active-site molecular docking of Nigellidine with nucleocapsid- Nsp2-Mpro of COVID-19 and to human IL1R-IL6R and strong antioxidant role of Nigella-sativa in experimental rats. J. Drug Target. https://doi.org/10.1080/1061186X.2020.1817040 (2020).

147. Patro, L. P. P., Sathyaseelan, C., Uttamrao, P. P. \& Rathinavelan, T. Global variation in SARS-CoV-2 proteome and its implication in pre-lockdown emergence and dissemination of 5 dominant SARS-CoV-2 clades. Infect. Genet. Evol. 93, 104973 (2021).

148. Flores-Alanis, A. et al. Molecular epidemiology surveillance of SARS-CoV-2: mutations and genetic diversity one year after emerging. Pathogens 10, 184 (2021).

149. Wang, R. et al. Analysis of SARS-CoV-2 mutations in the United States suggests presence of four substrains and novel variants. Commun. Biol. 4, 228 (2021).

150. Han, Y. S. et al. Papain-like protease 2 (PLP2) from severe acute respiratory syndrome coronavirus (SARS-CoV): expression, purification, characterization, and inhibition. Biochemistry 44, 10349-10359 (2005).

151. Shin, D. et al. Papain-like protease regulates SARS-CoV-2 viral spread and innate immunity. Nature 587, 657-662 (2020).

152. Frieman, M., Ratia, K., Johnston, R. E., Mesecar, A. D. \& Baric, R. S. Severe acute respiratory syndrome coronavirus papain-like protease ubiquitin-like domain and catalytic domain regulate antagonism of IRF3 and NF-kappaB signaling. J. Virol. 83, 6689-6705 (2009).

153. Harcourt, B. H. et al. Identification of severe acute respiratory syndrome coronavirus replicase products and characterization of papain-like protease activity. J. Virol. 78, 13600-13612 (2004).

154. Clementz, M. A. et al. Deubiquitinating and interferon antagonism activities of coronavirus papain-like proteases. J. Virol. 84, 4619-4629 (2010).

155. Chen, X., Chou, C. Y. \& Chang, G. G. Thiopurine analogue inhibitors of severe acute respiratory syndrome-coronavirus papain-like protease, a deubiquitinating and delSGylating enzyme. Antivir. Chem. Chemother. 19, 151-156 (2009).

156. Devaraj, S. G. et al. Regulation of IRF-3-dependent innate immunity by the papain-like protease domain of the severe acute respiratory syndrome coronavirus. J. Biol. Chem. 282, 32208-32221 (2007).

157. Lei, J., Kusov, Y. \& Hilgenfeld, R. Nsp3 of coronaviruses: structures and functions of a large multi-domain protein. Antivir. Res. 149, 58-74 (2018).

158. Yang, H. \& Rao, Z. Structural biology of SARS-CoV-2 and implications for therapeutic development. Nat. Rev. Microbiol. 19, 685-700 (2021).

159. Lavigne, M. et al. SARS-CoV-2 Nsp3 unique domain SUD interacts with guanine quadruplexes and G4-ligands inhibit this interaction. Nucleic Acids Res. 49, 7695-7712 (2021).

160. Hochstrasser, M. Origin and function of ubiquitin-like proteins. Nature $\mathbf{4 5 8}$, 422-429 (2009).

161. Serrano, $\mathrm{P}$. et al. Nuclear magnetic resonance structure of the $\mathrm{N}$-terminal domain of nonstructural protein 3 from the severe acute respiratory syndrome coronavirus. J. Virol. 81, 12049-12060 (2007).

162. Keane, S. C. \& Giedroc, D. P. Solution structure of mouse hepatitis virus (MHV) nsp3a and determinants of the interaction with MHV nucleocapsid $(\mathrm{N})$ protein. $J$. Virol. 87, 3502-3515 (2013).
163. Khan, M. T. et al. SARS-CoV-2 nucleocapsid and Nsp3 binding: an in silico study. Arch. Microbiol. 203, 59-66 (2021).

164. Schuller, M. et al. Fragment binding to the Nsp3 macrodomain of SARS-CoV-2 identified through crystallographic screening and computational docking. Sci. Adv. 7, eabf8711 (2021).

165. Fehr, A. et al. The conserved coronavirus macrodomain promotes virulence and suppresses the innate immune response during severe acute respiratory syndrome coronavirus infection. mBio 7, e01721 (2016).

166. Fehr, A. et al. The nsp3 macrodomain promotes virulence in mice with coronavirus-induced encephalitis. J. Virol. 89, 1523-1536 (2015).

167. Michalska, K. et al. Crystal structures of SARS-CoV-2 ADP-ribose phosphatase: from the apo form to ligand complexes. IUCrJ 7, 814-824 (2020).

168. Frick, D., Virdi, R., Vuksanovic, N., Dahal, N. \& Silvaggi, N. Molecular basis for ADPribose binding to the Mac1 domain of SARS-CoV-2 nsp3. Biochemistry 59, 2608-2615 (2020).

169. Alhammad, Y. et al. The SARS-CoV-2 conserved Macrodomain is a mono-ADPribosylhydrolase. J. Virol. 95, e01969-20 (2021).

170. James, D. et al. First-in-class chemical probes against poly(ADP-ribose) Glycohydrolase (PARG) inhibit DNA repair with differential pharmacology to Olaparib. ACS Chem. Biol. 11, 3179-3190 (2016).

171. Brosey, C. et al. Targeting SARS-CoV-2 Nsp3 macrodomain structure with insights from human poly (ADP-ribose) glycohydrolase (PARG) structures with inhibitors. Prog. Biophys. Mol. Biol. 163, 171-186 (2021).

172. Virdi, R. S. et al. Discovery of drug-like ligands for the Mac1 domain of SARS-CoV2 Nsp3. SLAS Discov. 25, 1162-1170 (2020).

173. Rut, W. et al. Activity profiling and crystal structures of inhibitor-bound SARSCoV-2 papain-like protease: a framework for anti-COVID-19 drug design. Sci. Adv. 6, eabd4596 (2020)

174. Fu, Z. et al. The complex structure of GRL0617 and SARS-CoV-2 PLpro reveals a hot spot for antiviral drug discovery. Nat. Commun. 12, 488 (2021).

175. Zhao, Y. et al. High-throughput screening identifies established drugs as SARS CoV-2 PLpro inhibitors. Protein Cell 12, 877-888 (2021).

176. Wojdyla, J. et al. Papain-like protease 1 from transmissible gastroenteritis virus: crystal structure and enzymatic activity toward viral and cellular substrates. $J$. Virol. 84, 10063-10073 (2010).

177. Guedes, I. A. et al. Drug design and repurposing with DockThor-VS web server focusing on SARS-CoV-2 therapeutic targets and their non-synonym variants. Sci. Rep. 11, 5543 (2021).

178. Báez-Santos, Y. M., St John, S. E. \& Mesecar, A. D. The SARS-coronavirus papainlike protease: structure, function and inhibition by designed antiviral compounds. Antivir. Res. 115, 21-38 (2015)

179. Barretto, N. et al. The papain-like protease of severe acute respiratory syndrome coronavirus has deubiquitinating activity. J. Virol. 79, 15189-15198 (2005).

180. Ratia, K. et al. A noncovalent class of papain-like protease/deubiquitinase inhibitors blocks SARS virus replication. Proc. Natl Acad. Sci. USA 105, 16119-16124 (2008).

181. Nakahara, T. et al. YM155, a novel small-molecule survivin suppressant, induces regression of established human hormone-refractory prostate tumor xenografts. Cancer Res. 67, 8014-8021 (2007).

182. Santerre, M., Arjona, S. P., Allen, C. N. S., Shcherbik, N. \& Sawaya, B. E. Why do SARS-CoV-2 NSPs rush to the ER? J. Neurol. 268, 2013-2022 (2021).

183. Hackstadt, T. et al. Disruption of the golgi apparatus and contribution of the Endoplasmic Reticulum to the SARS-CoV-2 replication complex. Viruses 13, 1798 (2021).

184. Chakraborty, J., Maity, A. \& Sarkar, H. A systematic drug repurposing approach to identify promising inhibitors from FDA-approved drugs against Nsp4 protein of SARS-CoV-2. J. Biomol. Struct. Dyn. https://doi.org/10.1080/07391102. 2021.2009033 (2021).

185. Chen, C. C. et al. Overview of antiviral drug candidates targeting coronaviral 3Clike main proteases. FEBS J. 288, 5089-5121 (2021).

186. Yang, H. T. et al. Design of wide-spectrum inhibitors targeting coronavirus main proteases. PLoS Biol. 3, e324 (2005).

187. Zhang, L. L. et al. Crystal structure of SARS-CoV-2 main protease provides a basis for design of improved a-ketoamide inhibitors. Science 368, 409-412 (2020).

188. Jin, Z. M. et al. Structural basis for the inhibition of SARS-CoV-2 main protease by antineoplastic drug carmofur. Nat. Struct. Mol. Biol. 27, 529-532 (2020).

189. Xiong, M. Y. et al. What coronavirus 3C-like protease tells us: from structure substrate selectivity, to inhibitor design. Med. Res. Rev. 41, 1965-1998 (2021).

190. Jin, Z. M. et al. Structure of $M^{\text {pro }}$ from SARS-CoV-2 and discovery of its inhibitors. Nature 582, 289-293 (2020).

191. Sacco, M. et al. Structure and inhibition of the SARS-CoV-2 main protease reveal strategy for developing dual inhibitors against $\mathrm{M}$ and cathepsin L. Sci. Adv. 6 , eabe0751 (2020).

192. Zhang, L. L. et al. a-Ketoamides as broad-spectrum inhibitors of coronavirus and enterovirus replication: structure-based design, synthesis, and activity assessment. J. Med. Chem. 63, 4562-4578 (2020). 
193. Ledford, H. COVID antiviral pills: what scientists still want to know. Nature $\mathbf{5 9 9}$, 358-359 (2021).

194. Zhao, Y. et al. Crystal structure of SARS-CoV-2 main protease in complex with protease inhibitor PF-07321332. Protein Cell. https://doi.org/10.1007/s13238021-00883-2 (2021).

195. Kandeel, M. \& Al-Nazawi, M. Virtual screening and repurposing of FDA approved drugs against COVID-19 main protease. Life Sci. 251, 117627 (2020).

196. Kumar, S. et al. Mammalian hybrid pre-autophagosomal structure HyPAS generates autophagosomes. Cell 184, 5950-5969 (2021).

197. Shemesh, M. et al. SARS-CoV-2 suppresses IFN $\beta$ production mediated by NSP1, $5,6,15$, ORF6 and ORF7b but does not suppress the effects of added interferon. PLoS Pathog. 17, e1009800 (2021).

198. Xia, H. J. et al. Evasion of type I interferon by SARS-CoV-2. Cell Rep. 33, 108234 (2020).

199. Vela, J. M. Repurposing sigma-1 receptor ligands for COVID-19 therapy? Front. Pharmacol. 11, 582310 (2020).

200. Pandey, P., Prasad, K., Prakash, A. \& Kumar, V. Insights into the biased activity of dextromethorphan and haloperidol towards SARS-CoV-2 NSP6: in silico binding mechanistic analysis. J. Mol. Med. (Berl.) 98, 1659-1673 (2020).

201. te Velthuis, A., van den Worm, S. \& Snijder, E. The SARS-coronavirus nsp7+nsp8 complex is a unique multimeric RNA polymerase capable of both de novo initiation and primer extension. Nucleic Acids Res. 40, 1737-1747 (2012).

202. Imbert, I. et al. A second, non-canonical RNA-dependent RNA polymerase in SARS coronavirus. EMBO J. 25, 4933-4942 (2014).

203. Hillen, H. et al. Structure of replicating SARS-CoV-2 polymerase. Nature $\mathbf{5 8 4}$, 154-156 (2020).

204. Biswal, M. et al. Two conserved oligomer interfaces of Nsp7 and Nsp8 underpin the dynamic assembly of SARS-CoV-2 RdRp. Nucleic Acids Res. 49, 5956-5966 (2021).

205. Johnson, M., Jaudzems, K. \& Wüthrich, K. NMR structure of the SARS-CoV nonstructural protein 7 in solution at pH 6.5. J. Mol. Biol. 402, 619-628 (2010).

206. Zhang, C. H., Li, L., He, J., Chen, C. \& Su, D. Nonstructural protein 7 and 8 complexes of SARS-CoV-2. Protein Sci. 30, 873-881 (2021).

207. Chen, J. et al. Structural basis for helicase-polymerase coupling in the SARS-CoV2 replication-transcription complex. Cell 182, 1560-1573 (2020).

208. Yan, L. M. et al. Architecture of a SARS-CoV-2 mini replication and transcription complex. Nat. Commun. 11, 5874 (2020).

209. Wilamowski, M. et al. Transient and stabilized complexes of Nsp7, Nsp8, and Nsp12 in SARS-CoV-2 replication. Biophys. J. 120, 3152-3165 (2021).

210. Zhai, Y. J. et al. Insights into SARS-CoV transcription and replication from the structure of the nsp7-nsp8 hexadecamer. Nat. Struct. Mol. Biol. 12, 980-986 (2005).

211. Egloff, M. et al. The severe acute respiratory syndrome-coronavirus replicative protein nsp9 is a single-stranded RNA-binding subunit unique in the RNA virus world. Proc. Natl Acad. Sci. USA 101, 3792-3796 (2004).

212. Frieman, M. et al. Molecular determinants of severe acute respiratory syndrome coronavirus pathogenesis and virulence in young and aged mouse models of human disease. J. Virol. 86, 884-897 (2012).

213. Littler, D. R., Gully, B. S., Colson, R. N. \& Rossjohn, J. Crystal structure of the SARSCoV-2 non-structural protein 9, Nsp9. iScience 23, 101258 (2020).

214. Zhang, C. H. et al. Structural basis for the multimerization of nonstructural protein nsp9 from SARS-CoV-2. Mol. Biomed. 1, 5 (2020).

215. Sutton, G. et al. The nsp9 replicase protein of SARS-coronavirus, structure and functional insights. Structure 12, 341-353 (2004).

216. Littler, D. et al. Binding of a pyrimidine RNA base-mimic to SARS-CoV-2 nonstructural protein 9. J. Biol. Chem. 297, 101018 (2021).

217. $\mathrm{Hu}$, T. et al. Structural basis for dimerization and RNA binding of avian infectious bronchitis virus nsp9. Protein Sci. 26, 1037-1048 (2017).

218. Satheesh, D., Rajendran, A. \& Chithra, K. Protein-ligand binding interactions of imidazolium salts with SARS CoV-2. Heliyon 6, e05544 (2020).

219. Sadia, A., Azam, M. \& Basra, M. A. R. Teicoplanin is a potential inhibitor of SARS CoV-2 replication enzymes: a docking study. Asian Pac. J. Trop. Biomed. 10, 563-568 (2020).

220. Donaldson, E., Sims, A., Graham, R., Denison, M. \& Baric, R. Murine hepatitis virus replicase protein nsp10 is a critical regulator of viral RNA synthesis. J. Virol. 81, 6356-6368 (2007).

221. Decroly, E., Ferron, F., Lescar, J. \& Canard, B. Conventional and unconventional mechanisms for capping viral mRNA. Nat. Rev. Microbiol. 10, 51-65 (2011).

222. Yan, L. M. et al. Cryo-EM structure of an extended SARS-CoV-2 replication and transcription complex reveals an intermediate state in cap synthesis. Cell 184, 184-193 (2021).

223. Bouvet, M. et al. In vitro reconstitution of SARS-coronavirus mRNA cap methylation. PLoS Pathog. 6, e1000863 (2010).

224. Chen, Y. et al. Functional screen reveals SARS coronavirus nonstructural protein nsp14 as a novel cap N7 methyltransferase. Proc. Natl Acad. Sci. USA 106, 3484-3489 (2009).
225. Romano, M., Ruggiero, A., Squeglia, F., Maga, G. \& Berisio, R. A structural view of SARS-CoV-2 RNA replication machinery: RNA synthesis, proofreading and final capping. Cells 9, 1267 (2020)

226. Ke, M. et al. Short peptides derived from the interaction domain of SARS coronavirus nonstructural protein nsp10 can suppress the 2'-O-methyltransferase activity of nsp10/nsp16 complex. Virus Res. 167, 322-328 (2012).

227. Wang, Y. et al. Coronavirus nsp10/nsp16 methyltransferase can be targeted by nsp10-derived peptide in vitro and in vivo to reduce replication and pathogenesis. J. Virol. 89, 8416-8427 (2015).

228. Su, D. et al. Dodecamer structure of severe acute respiratory syndrome coronavirus nonstructural protein nsp10. J. Virol. 80, 7902-7908 (2006).

229. Kirchdoerfer, R. \& Ward, A. Structure of the SARS-CoV nsp12 polymerase bound to nsp7 and nsp8 co-factors. Nat. Commun. 10, 2342 (2019).

230. Wang, Q. et al. Structural basis for RNA replication by the SARS-CoV-2 polymerase. Cell 182, 417-428 (2020).

231. Yan, L. M. et al. Coupling of N7-methyltransferase and $3^{\prime}-5$ ' exoribonuclease with SARS-CoV-2 polymerase reveals mechanisms for capping and proofreading. Cell 184, 3474-3485 (2021).

232. Lehmann, K. et al. Discovery of an essential nucleotidylating activity associated with a newly delineated conserved domain in the RNA polymerase-containing protein of all nidoviruses. Nucleic Acids Res. 43, 8416-8434 (2015).

233. Gao, Y. et al. Structure of the RNA-dependent RNA polymerase from COVID-19 virus. Science 368, 779-782 (2020).

234. Yin, W. C. et al. Structural basis for inhibition of the RNA-dependent RNA polymerase from SARS-CoV-2 by remdesivir. Science 368, 1499-1504 (2020).

235. Al-Tawfiq, J. A., Momattin, H., Dib, J. \& Memish, Z. A. Ribavirin and interferon therapy in patients infected with the Middle East respiratory syndrome coronavirus: an observational study. Int. J. Infec Dis. 20, 42-46 (2014).

236. De Clercq, E. New nucleoside analogues for the treatment of hemorrhagic fever virus infections. Chem. Asian J. 14, 3962-3968 (2019).

237. Gane, E. et al. Nucleotide polymerase inhibitor sofosbuvir plus ribavirin for hepatitis C. N. Engl. J. Med. 368, 34-44 (2013).

238. Sheahan, T. et al. An orally bioavailable broad-spectrum antiviral inhibits SARSCoV-2 in human airway epithelial cell cultures and multiple coronaviruses in mice. Sci. Transl. Med. 12, eabb5883 (2020).

239. Elfiky, A. Ribavirin, Remdesivir, Sofosbuvir, Galidesivir, and Tenofovir against SARS-CoV-2 RNA dependent RNA polymerase (RdRp): a molecular docking study. Life Sci. 253, 117592 (2020).

240. Wang, M. L. et al. Remdesivir and chloroquine effectively inhibit the recently emerged novel coronavirus (2019-nCoV) in vitro. Cell Res. 30, 269-271 (2020).

241. Tchesnokov, E., Feng, J., Porter, D. \& Götte, M. Mechanism of inhibition of ebola virus RNA-dependent RNA polymerase by remdesivir. Viruses 11, 326 (2019).

242. Sheahan, T. et al. Broad-spectrum antiviral GS-5734 inhibits both epidemic and zoonotic coronaviruses. Sci. Transl. Med. 9, eaal3653 (2017).

243. Bravo, J., Dangerfield, T., Taylor, D. \& Johnson, K. Remdesivir is a delayed translocation inhibitor of SARS-CoV-2 replication. Mol. Cell 81, 1548-1552 (2021).

244. Kokic, G. et al. Mechanism of SARS-CoV-2 polymerase stalling by remdesivir. Nat. Commun. 12, 279 (2021).

245. Tchesnokov, E. P. et al. Template-dependent inhibition of coronavirus RNAdependent RNA polymerase by remdesivir reveals a second mechanism of action. J. Biol. Chem. 295, 16156-16165 (2020).

246. Kabinger, F. et al. Mechanism of molnupiravir-induced SARS-CoV-2 mutagenesis. Nat. Struct. Mol. Biol. 28, 740-746 (2021).

247. Zoltner, M. et al. Suramin exposure alters cellular metabolism and mitochondrial energy production in African trypanosomes. J. Biol. Chem. 295, 8331-8347 (2020).

248. Yin, W. C. et al. Structural basis for inhibition of the SARS-CoV-2 RNA polymerase by suramin. Nat. Struct. Mol. Biol. 28, 319-325 (2021).

249. Tanner, J. A., Watt, R. M., Chai, Y. B., Lu, L. Y. \& Huang, J. D. The severe acute respiratory syndrome (SARS) coronavirus NTPase/helicase belongs to a distinct class of $5^{\prime}$ to $3^{\prime}$ viral helicases. J. Biol. Chem. 278, 39578-39582 (2003).

250. Lee, N. et al. Cooperative translocation enhances the unwinding of duplex DNA by SARS coronavirus helicase nsp13. Nucleic Acids Res. 38, 7626-7636 (2010).

251. Ivanov, K. et al. Multiple enzymatic activities associated with severe acute respiratory syndrome coronavirus helicase. J. Virol. 78, 5619-5632 (2004).

252. Ivanov, K. \& Ziebuhr, J. Human coronavirus $229 \mathrm{E}$ nonstructural protein 13: characterization of duplex-unwinding, nucleoside triphosphatase, and RNA $5^{\prime}-$ triphosphatase activities. J. Virol. 78, 7833-7838 (2004).

253. Lehmann, K. C., Snijder, E. J., Posthuma, C. C. \& Gorbalenya, A. E. What we know but do not understand about nidovirus helicases. Virus Res. 202, 12-32 (2015).

254. Hao, W. et al. Crystal structure of Middle East respiratory syndrome coronavirus helicase. PLoS Pathog. 13, e1006474 (2017).

255. Singleton, M., Dillingham, M. \& Wigley, D. Structure and mechanism of helicases and nucleic acid translocases. Annu. Rev. Biochem. 76, 23-50 (2007).

256. Adedeji, A. et al. Mechanism of nucleic acid unwinding by SARS-CoV helicase. PLOS ONE 7, e36521 (2012). 
257. Malone, B. et al. Structural basis for backtracking by the SARS-CoV-2 replicationtranscription complex. Proc. Natl Acad. Sci. USA 118, e2102516118 (2021).

258. Snijder, E., Decroly, E. \& Ziebuhr, J. The nonstructural proteins directing coronavirus RNA synthesis and processing. Adv. Virus Res. 96, 59-126 (2016).

259. Nudler, E. RNA polymerase backtracking in gene regulation and genome instability. Cell 149, 1438-1445 (2012).

260. Cheung, A. \& Cramer, P. Structural basis of RNA polymerase II backtracking, arrest and reactivation. Nature 471, 249-253 (2011).

261. Denison, M., Graham, R., Donaldson, E., Eckerle, L. \& Baric, R. Coronaviruses: an RNA proofreading machine regulates replication fidelity and diversity. RNA Biol. 8, 270-279 (2011)

262. Smith, E., Blanc, H., Surdel, M., Vignuzzi, M. \& Denison, M. Coronaviruses lacking exoribonuclease activity are susceptible to lethal mutagenesis: evidence for proofreading and potential therapeutics. PLoS Pathog. 9, e1003565 (2013).

263. Shannon, A. et al. Rapid incorporation of Favipiravir by the fast and permissive viral RNA polymerase complex results in SARS-CoV-2 lethal mutagenesis. Nat. Commun. 11, 4682 (2020).

264. Ma, Y. Y. et al. Structural basis and functional analysis of the SARS coronavirus nsp14-nsp10 complex. Proc. Natl Acad. Sci. USA 112, 9436-9441 (2015).

265. Becares, M. et al. Mutagenesis of coronavirus nsp14 reveals its potential role in modulation of the innate immune response. J. Virol. 90, 5399-5414 (2016)

266. Hastie, K., Kimberlin, C., Zandonatti, M., MacRae, I. \& Saphire, E. Structure of the Lassa virus nucleoprotein reveals a dsRNA-specific $3^{\prime}$ to $5^{\prime}$ exonuclease activity essential for immune suppression. Proc. Natl Acad. Sci. USA 108, 2396-2401 (2011).

267. Hsu, J., Laurent-Rolle, M., Pawlak, J., Wilen, C. \& Cresswell, P. Translational shutdown and evasion of the innate immune response by SARS-CoV-2 Nsp14 protein. Proc. Natl Acad. Sci. USA 118, e2101161118 (2021).

268. Corman, V., Muth, D., Niemeyer, D. \& Drosten, C. Hosts and sources of endemic human coronaviruses. Adv. Virus Res. 100, 163-188 (2018).

269. Bouvet, M. et al. RNA 3 '-end mismatch excision by the severe acute respiratory syndrome coronavirus nonstructural protein nsp10/nsp14 exoribonuclease complex. Proc. Natl Acad. Sci. USA 109, 9372-9377 (2012).

270. Lin, S. et al. Crystal structure of SARS-CoV-2 nsp10 bound to nsp14-ExoN domain reveals an exoribonuclease with both structural and functional integrity. Nucleic Acids Res. 49, 5382-5392 (2021).

271. Tahir, M. Coronavirus genomic nsp14-ExoN, structure, role, mechanism, and potential application as a drug target. J. Med. Virol. 93, 4258-4264 (2021).

272. Decroly, E. et al. Crystal structure and functional analysis of the SARS coronavirus RNA cap 2'-O-methyltransferase nsp10/nsp16 complex. PLoS Pathog. 7, e1002059 (2011).

273. Ahmed-Belkacem, R. et al. Synthesis of adenine dinucleosides SAM analogs as specific inhibitors of SARS-CoV nsp14 RNA cap guanine-N7-methyltransferase. Eur. J. Med. Chem. 201, 112557 (2020).

274. Chen, Y. et al. Structure-function analysis of severe acute respiratory syndrome coronavirus RNA cap guanine-N7-methyltransferase. J. Virol. 87, 6296-6305 (2013).

275. Liu, C. et al. Structural basis of mismatch recognition by a SARS-CoV-2 proofreading enzyme. Science 373, 1142-1146 (2021).

276. Ferron, F. et al. Structural and molecular basis of mismatch correction and ribavirin excision from coronavirus RNA. Proc. Natl Acad. Sci. USA 115, 162-171 (2018).

277. Robson, F. et al. Coronavirus RNA proofreading: molecular basis and therapeutic targeting. Mol. Cell 79, 710-727 (2020).

278. Eastman, R. et al. Remdesivir: a review of its discovery and development leading to emergency use authorization for treatment of COVID-19. ACS Cent. Sci. 6, 672-683 (2020)

279. Jockusch, S. et al. Sofosbuvir terminated RNA is more resistant to SARS-CoV-2 proofreader than RNA terminated by Remdesivir. Sci. Rep. 10, 16577 (2020).

280. Khater, S. et al. Combining SARS-CoV-2 proofreading exonuclease and RNAdependent RNA polymerase inhibitors as a strategy to combat COVID-19: a high-throughput in silico screening. Front. Microbiol. 12, 647693 (2021).

281. Sargsyan, K., Lin, C. C., Chen, T., Grauffel, C. \& Lim, C. Multi-targeting of functional cysteines in multiple conserved SARS-CoV-2 domains by clinically safe $\mathrm{Zn}$ ejectors. Chem. Sci. 11, 9904-9909 (2020).

282. Chen, T. et al. Synergistic inhibition of SARS-CoV-2 replication using disulfiram/ ebselen and remdesivir. ACS Pharmacol. Transl. Sci. 4, 898-907 (2021).

283. He, R. T. et al. Potent and selective inhibition of SARS coronavirus replication by aurintricarboxylic acid. Biochem. Biophys. Res. Commun. 320, 1199-1203 (2004).

284. Devkota, K. et al. Probing the SAM binding site of SARS-CoV-2 Nsp14 in vitro using SAM competitive inhibitors guides developing selective bisubstrate inhibitors. SLAS Discov. 26, 1200-1211 (2021).

285. Graham, R. L. et al. A live, impaired-fidelity coronavirus vaccine protects in an aged, immunocompromised mouse model of lethal disease. Nat. Med. 18, 1820-1826 (2012).
286. Menachery, V. et al. Attenuation and restoration of severe acute respiratory syndrome coronavirus mutant lacking 2'-O-methyltransferase activity. J. Virol. 88, 4251-4264 (2014).

287. Lu, Y. J. et al. Porcine epidemic diarrhea virus deficient in RNA cap Guanine-N-7 methylation is attenuated and induces higher type I and III interferon responses. J. Virol. 94, e00447-20 (2020).

288. Zhang, L. Q. et al. Structural and biochemical characterization of endoribonuclease Nsp15 encoded by middle east respiratory syndrome coronavirus. J. Virol. 92, e00893-18 (2018).

289. Kim, Y. et al. Tipiracil binds to uridine site and inhibits Nsp15 endoribonuclease NendoU from SARS-CoV-2. Commun. Biol. 4, 193 (2021).

290. Bhardwaj, K., Guarino, L. \& Kao, C. The severe acute respiratory syndrome coronavirus Nsp15 protein is an endoribonuclease that prefers manganese as a cofactor. J. Virol. 78, 12218-12224 (2004)

291. Deng, X. F. et al. Coronavirus nonstructural protein 15 mediates evasion of dsRNA sensors and limits apoptosis in macrophages. Proc. Natl Acad. Sci. USA 114, 4251-4260 (2017).

292. Hackbart, M., Deng, X. \& Baker, S. Coronavirus endoribonuclease targets viral polyuridine sequences to evade activating host sensors. Proc. Natl Acad. Sci. USA 117, 8094-8103 (2020).

293. Deng, X. F. et al. Coronavirus endoribonuclease activity in porcine epidemic diarrhea virus suppresses type I and Type III interferon responses. J. Virol. 93 e02000-e02018 (2019).

294. Deng, X. F. \& Baker, S. An "old" protein with a new story: coronavirus endor ibonuclease is important for evading host antiviral defenses. Virology 517, 157-163 (2018).

295. Pillon, M. et al. Cryo-EM structures of the SARS-CoV-2 endoribonuclease Nsp15 reveal insight into nuclease specificity and dynamics. Nat. Commun. 12, 636 (2021).

296. Kim, Y. et al. Crystal structure of Nsp15 endoribonuclease NendoU from SARSCoV-2. Protein Sci. 29, 1596-1605 (2020).

297. Frazier, M. et al. Characterization of SARS2 Nsp15 nuclease activity reveals it's mad about U. Nucleic Acids Res. 49, 10136-10419 (2021).

298. Frazier, M. N. et al. Characterization of SARS2 Nsp15 nuclease activity reveals it's mad about U. Nucleic Acids Res. 49, 10136-10149 (2021).

299. Joseph, J. et al. Crystal structure of a monomeric form of severe acute respiratory syndrome coronavirus endonuclease nsp 15 suggests a role for hexamerization as an allosteric switch. J. Virol. 81, 6700-6708 (2007).

300. Bhardwaj, K. et al. Structural and functional analyses of the severe acute respiratory syndrome coronavirus endoribonuclease Nsp15. J. Biol. Chem. 283, 3655-3664 (2008)

301. Guo, F., Li, Q. \& Zhou, C. Synthesis and biological applications of fluoro-modified nucleic acids. Org. Biomol. Chem. 15, 9552-9565 (2017).

302. Kish, T. \& Uppal, P. Trifluridine/Tipiracil (Lonsurf) for the treatment of metastatic colorectal cancer. P T 41, 314-325 (2016).

303. Li, Y. et al. Nsp9 and Nsp10 contribute to the fatal virulence of highly pathogenic porcine reproductive and respiratory syndrome virus emerging in China. PLoS Pathog. 10, e1004216 (2014).

304. Aouadi, W. et al. Binding of the methyl donor S-Adenosyl-L-methionine to middle east respiratory syndrome coronavirus 2'-O-methyltransferase nsp16 promotes recruitment of the allosteric activator nsp10. J. Virol. 91, e02217-16 (2017).

305. Ramanathan, A., Robb, G. \& Chan, S. H. mRNA capping: biological functions and applications. Nucleic Acids Res. 44, 7511-7526 (2016).

306. Chen, Y. et al. Biochemical and structural insights into the mechanisms of SARS coronavirus RNA ribose 2'-O-methylation by nsp $16 / \mathrm{nsp} 10$ protein complex. PLoS Pathog. 7, e1002294 (2011).

307. Viswanathan, T. et al. Structural basis of RNA cap modification by SARS-CoV-2 Nat. Commun. 11, 3718 (2020).

308. Rosas-Lemus, M. et al. High-resolution structures of the SARS-CoV-2 2'-Omethyltransferase reveal strategies for structure-based inhibitor design. Sci. Signal. 13, eabe1202 (2020).

309. Wilamowski, M. et al. 2'-O methylation of RNA cap in SARS-CoV-2 captured by serial crystallography. Proc. Natl Acad. Sci. USA 118, e2100170118 (2021).

310. Bollati, M. et al. Recognition of RNA cap in the Wesselsbron virus NS5 methyltransferase domain: implications for RNA-capping mechanisms in Flavivirus. J. Mol. Biol. 385, 140-152 (2009).

311. Bujnicki, J. \& Rychlewski, L. In silico identification, structure prediction and phylogenetic analysis of the 2'-O-ribose (cap 1) methyltransferase domain in the large structural protein of ssRNA negative-strand viruses. Protein Eng. 15 101-108 (2002)

312. Chang, L. J. \& Chen, T. H. NSP16 2'-O-MTase in coronavirus pathogenesis: possible prevention and treatments strategies. Viruses 13, 538 (2021).

313. Redondo, N., Zaldívar-López, S., Garrido, J. \& Montoya, M. SARS-CoV-2 accessory proteins in viral pathogenesis: knowns and unknowns. Front. Immunol. 12, 708264 (2021). 
314. Rohaim, M., El Naggar, R., Clayton, E. \& Munir, M. Structural and functional insights into non-structural proteins of coronaviruses. Micro. Pathog. 150, 104641 (2021).

315. Liu, D., Fung, T., Chong, K., Shukla, A. \& Hilgenfeld, R. Accessory proteins of SARSCoV and other coronaviruses. Antivir. Res. 109, 97-109 (2014).

316. McBride, R. \& Fielding, B. The role of severe acute respiratory syndrome (SARS)coronavirus accessory proteins in virus pathogenesis. Viruses 4, 2902-2923 (2012).

317. Tan, Y., Lim, S. \& Hong, W. Understanding the accessory viral proteins unique to the severe acute respiratory syndrome (SARS) coronavirus. Antivir. Res. 72, 78-88 (2006).

318. Freundt, E. et al. The open reading frame 3a protein of severe acute respiratory syndrome-associated coronavirus promotes membrane rearrangement and cell death. J. Virol. 84, 1097-1109 (2010).

319. Issa, E., Merhi, G., Panossian, B., Salloum, T. \& Tokajian, S. SARS-CoV-2 and ORF3a: nonsynonymous mutations, functional domains, and viral pathogenesis. $m S y s-$ tems 5, e00266-20 (2020).

320. Andersen, K., Rambaut, A., Lipkin, W., Holmes, E. \& Garry, R. The proximal origin of SARS-CoV-2. Nat. Med. 26, 450-452 (2020).

321. Siu, K. et al. Severe acute respiratory syndrome coronavirus ORF3a protein activates the NLRP3 inflammasome by promoting TRAF3-dependent ubiquitination of ASC. FASEB J. 33, 8865-8877 (2019).

322. Ren, Y. J. et al. The ORF3a protein of SARS-CoV-2 induces apoptosis in cells. Cell Mol. Immunol. 17, 881-883 (2020).

323. Miao, G. et al. ORF3a of the COVID-19 virus SARS-CoV-2 blocks HOPS complexmediated assembly of the SNARE complex required for autolysosome formation. Dev. Cell 56, 427-442 (2021).

324. McClenaghan, C., Hanson, A., Lee, S. \& Nichols, C. Coronavirus proteins as ion channels: current and potential research. Front. Immunol. 11, 573339 (2020).

325. Azad, G. \& Khan, P. Variations in Orf3a protein of SARS-CoV-2 alter its structure and function. Biochem. Biophys. Rep. 26, 100933 (2021).

326. Kern, D. M. et al. Cryo-EM structure of the SARS-CoV-2 ORF3a in lipid nanodiscs. Nat. Struct. Mol. Biol. 28, 573-582 (2021).

327. Lu, W. et al. Severe acute respiratory syndrome-associated coronavirus 3a protein forms an ion channel and modulates virus release. Proc. Natl Acad. Sci. USA 103, 12540-12545 (2006).

328. Minakshi, R. \& Padhan, K. The YXXФ motif within the severe acute respiratory syndrome coronavirus (SARS-CoV) 3a protein is crucial for its intracellular transport. Virol. J. 11, 75 (2014).

329. Gordon, D. et al. A SARS-CoV-2 protein interaction map reveals targets for drug repurposing. Nature 583, 459-468 (2020).

330. Lee, T. S. \& Chau, L. Y. Heme oxygenase-1 mediates the anti-inflammatory effect of interleukin-10 in mice. Nat. Med. 8, 240-246 (2002).

331. Lv, J. et al. Heme oxygenase-1 protects airway epithelium against apoptosis by targeting the proinflammatory NLRP3-RXR axis in asthma. J. Biol. Chem. 293, 18454-18465 (2018).

332. Origassa, C. \& Câmara, N. Cytoprotective role of heme oxygenase- 1 and heme degradation derived end products in liver injury. World J. Hepatol. 5, 541-549 (2013).

333. Grifoni, A. et al. Targets of T cell responses to SARS-CoV-2 coronavirus in humans with COVID-19 disease and unexposed individuals. Cell 181, 1489-1501 (2020).

334. Oja, A. E. et al. Divergent SARS-CoV-2-specific T and B cell responses in severe but not mild COVID-19 patients. Eur. J. Immunol. 50, 1998-2012 (2020).

335. Zhou, Z. L. et al. Structural insight reveals SARS-CoV-2 ORF7a as an immunomodulating factor for human CD14 monocytes. iScience 24, 102187 (2021).

336. Nelson, C., Pekosz, A., Lee, C., Diamond, M. \& Fremont, D. Structure and intracellular targeting of the SARS-coronavirus Orf7a accessory protein. Structure 13, 75-85 (2005).

337. Hachim, A. et al. ORF8 and ORF3b antibodies are accurate serological markers of early and late SARS-CoV-2 infection. Nat. Immunol. 21, 1293-1301 (2020).

338. Su, C. M., Wang, L. Y. \& Yoo, D. W. Activation of NF-KB and induction of proinflammatory cytokine expressions mediated by ORF7a protein of SARS-CoV-2. Sci. Rep. 11, 13464 (2021).

339. Li, J. Y. et al. The ORF6, ORF8 and nucleocapsid proteins of SARS-CoV-2 inhibit type I interferon signaling pathway. Virus Res. 286, 198074 (2020).

340. Cao, Z. G. et al. Ubiquitination of SARS-CoV-2 ORF7a promotes antagonism of interferon response. Cell Mol. Immunol. 18, 746-748 (2021).

341. Nemudryi, A. et al. SARS-CoV-2 genomic surveillance identifies naturally occurring truncation of ORF7a that limits immune suppression. Cell Rep. 35, 109197 (2021)

342. Chen, S. Y. et al. Extended ORF8 gene region is valuable in the epidemiological investigation of severe acute respiratory syndrome-similar coronavirus. J. Infect. Dis. 222, 223-233 (2020).

343. Cui, J., Li, F. \& Shi, Z. L. Origin and evolution of pathogenic coronaviruses. Nat. Rev. Microbiol. 17, 181-192 (2019).
344. Pereira, F. Evolutionary dynamics of the SARS-CoV-2 ORF8 accessory gene. Infect. Genet. Evol. 85, 104525 (2020).

345. Chen, $C$. Y. et al. Open reading frame 8 a of the human severe acute respiratory syndrome coronavirus not only promotes viral replication but also induces apoptosis. J. Infect. Dis. 196, 405-415 (2007).

346. Zhang, Y. W. et al. The ORF8 protein of SARS-CoV-2 mediates immune evasion through down-regulating MHC-I. Proc. Natl Acad. Sci. USA 118, e2024202118 (2021).

347. Wong, $\mathrm{H}$. H. et al. Accessory proteins $8 \mathrm{~b}$ and $8 \mathrm{ab}$ of severe acute respiratory syndrome coronavirus suppress the interferon signaling pathway by mediating ubiquitin-dependent rapid degradation of interferon regulatory factor 3. Virology 515, 165-175 (2018).

348. Yuen, C. K. et al. SARS-CoV-2 nsp13, nsp14, nsp15 and orf6 function as potent interferon antagonists. Emerg. Microbes Infect. 9, 1418-1428 (2020).

349. Zinzula, L. Lost in deletion: The enigmatic ORF8 protein of SARS-CoV-2. Biochem. Biophys. Res. Commun. 538, 116-124 (2021).

350. Gordon, D. et al. Comparative host-coronavirus protein interaction networks reveal pan-viral disease mechanisms. Science 370, 1181-1206 (2020).

351. Flower, T. et al. Structure of SARS-CoV-2 ORF8, a rapidly evolving immune evasion protein. Proc. Natl Acad. Sci. USA 118, e2021785118 (2021).

352. Kopecky-Bromberg, S., Martínez-Sobrido, L., Frieman, M., Baric, R. \& Palese, P. Severe acute respiratory syndrome coronavirus open reading frame (ORF) $3 \mathrm{~b}$, ORF 6, and nucleocapsid proteins function as interferon antagonists. J. Virol. 81, 548-557 (2007).

353. Gao, X. P. et al. Crystal structure of SARS-CoV-2 Orf9b in complex with human TOM70 suggests unusual virus-host interactions. Nat. Commun. 12, 2843 (2021).

354. Meier, $C$. et al. The crystal structure of ORF-9b, a lipid binding protein from the SARS coronavirus. Structure 14, 1157-1165 (2006).

355. Young, J., Hoogenraad, N. \& Hartl, F. Molecular chaperones Hsp90 and Hsp70 deliver preproteins to the mitochondrial import receptor Tom70. Cell 112, 41-50 (2003).

356. Liu, X. Y., Wei, B., Shi, H. X., Shan, Y. F. \& Wang, C. Tom70 mediates activation of interferon regulatory factor 3 on mitochondria. Cell Res. 20, 994-1011 (2010).

357. Kreimendahl, S. \& Rassow, J. The mitochondrial outer membrane protein Tom70-mediator in protein traffic, membrane contact sites and innate immunity. Int. J. Mol. Sci. 21, 7262 (2020).

358. Jiang, H. W. et al. SARS-CoV-2 proteome microarray for global profiling of COVID-19 specific IgG and IgM responses. Nat. Commun. 11, 3581 (2020).

359. Wang, M. F. et al. SARS-CoV-2 RdRp is a versatile enzyme with proofreading activity and ability to incorporate NHC into RNA by using diphosphate form molnupiravir as a substrate. bioRxiv https://doi.org/10.1101/2021.11.15.468737 (2021).

360. Salleh, M. Z., Derrick, J. P. \& Deris, Z. Z. Structural evaluation of the Spike glycoprotein variants on SARS-CoV-2 transmission and immune evasion. Int. J. Mol. Sci. 22, 7425 (2021).

361. Kim, Y. J., Jang, U. S., Soh, S. M., Lee, J. Y. \& Lee, H. R. The impact on infectivity and neutralization efficiency of SARS-CoV-2 lineage B.1.351 pseudovirus. Viruses 13, 633 (2021)

362. Ullrich, S., Ekanayake, K. B., Otting, G. \& Nitsche, C. Main protease mutants of SARS-CoV-2 variants remain susceptible to PF-07321332. bioRxiv https://doi.org/ 10.1101/2021.11.28.470226 (2021)

363. Hoffmann, M. et al. SARS-CoV-2 variants B.1.351 and P.1 escape from neutralizing antibodies. Cell 184, 2384-2393 (2021).

364. Arora, P. et al. Delta variant (B.1.617.2) sublineages do not show increased neutralization resistance. Cell Mol. Immunol. 18, 2557-2559 (2021).

365. ACTIV-3/Therapeutics for inpatients with COVID-19 (TICO) study Group. Efficacy and safety of two neutralising monoclonal antibody therapies, sotrovimab and BRII-196 plus BRII-198, for adults hospitalised with COVID-19 (TICO): a randomised controlled trial. Lancet Infect. Dis. https://doi.org/10.1016/s1473-3099(21) 00751-9 (2021).

366. Jayk Bernal, A. et al. Molnupiravir for oral treatment of COVID-19 in nonhospitalized patients. N. Engl. J. Med. https://doi.org/10.1056/NEJMoa2116044 (2021).

367. Bennett, R. P. et al. Sangivamycin is highly effective against SARS-CoV-2 in vitro and has favorable drug properties. $J \mathrm{Cl}$ Insight 7, e153165 (2021).

368. Gil, C. et al. COVID-19: Drug targets and potential treatments. J. Med. Chem. 63, 12359-12386 (2020).

369. Barton, C. et al. Activity of and effect of subcutaneous treatment with the broadspectrum antiviral lectin griffithsin in two laboratory rodent models. Antimicrob. Agents Chemother. 58, 120-127 (2014).

370. Hickson, S. E., Margineantu, D., Hockenbery, D. M., Simon, J. A. \& Geballe, A. P. Inhibition of vaccinia virus replication by nitazoxanide. Virology $\mathbf{5 1 8}, \mathbf{3 9 8}-405$ (2018).

371. Mycroft-West, C. J. et al. Heparin inhibits cellular invasion by SARS-CoV-2: structural dependence of the interaction of the Spike S1 receptor-binding domain with heparin. Thromb. Haemost. 120, 1700-1715 (2020). 
372. Straughn, A. R. \& Kakar, S. S. Withaferin A: a potential therapeutic agent against COVID-19 infection. J. Ovarian Res. 13, 79 (2020).

373. Zhao, T. Y. \& Patankar, N. A. Tetracycline as an inhibitor to the SARS-CoV-2. J. Cell Biochem. 122, 752-759 (2021).

374. Zhang, Y. \& Tang, L. V. Overview of targets and potential drugs of SARS-CoV-2 according to the viral replication. J. Proteome Res. 20, 49-59 (2021).

375. Wu, C. R. et al. Analysis of therapeutic targets for SARS-CoV-2 and discovery of potential drugs by computational methods. Acta Pharm. Sin. B 10, 766-788 (2020).

376. Choudhury, S., Moulick, D., Borah, A., Saikia, P. \& Mazumder, M. K. In search of drugs to alleviate suppression of the host's innate immune responses against SARS-CoV-2 using a molecular modeling approach. In Silico Pharmacol. 9, 26 (2021).

377. Amin, S. A., Banerjee, S., Ghosh, K., Gayen, S. \& Jha, T. Protease targeted COVID19 drug discovery and its challenges: Insight into viral main protease (Mpro) and papain-like protease (PLpro) inhibitors. Bioorg. Med. Chem. 29, 115860-115860 (2021).

378. Enmozhi, S. K., Raja, K., Sebastine, I. \& Joseph, J. Andrographolide as a potential inhibitor of SARS-CoV-2 main protease: an in silico approach. J. Biomol. Struct. Dyn. 39, 3092-3098 (2021)

379. Khanna, K. et al. Herbal immune-boosters: substantial warriors of pandemic COVID-19 battle. Phytomedicine 85, 153361 (2021).

380. Dong, X. Q., Tian, Z. X., Shen, C. W. \& Zhao, C. R. An overview of potential therapeutic agents to treat COVID-19. Biosci. Trends 14, 318-327 (2020).

381. Kim, D. J. et al. Vitamin B12 and folic acid alleviate symptoms of nutritional deficiency by antagonizing aryl hydrocarbon receptor. Proc. Natl Acad. Sci. USA 117, 15837-15845 (2020).

382. Wang, X. Q. \& Guan, Y. F. COVID-19 drug repurposing: a review of computational screening methods, clinical trials, and protein interaction assays. Med. Res. Rev. 41, 5-28 (2021).

383. Molavi, Z. et al. Identification of FDA approved drugs against SARS-CoV-2 RNA dependent RNA polymerase (RdRp) and 3-chymotrypsin-like protease (3CLpro), drug repurposing approach. Biomed. Pharmacother. 138, 111544 (2021).

384. Mohamed, K., Yazdanpanah, N., Saghazadeh, A. \& Rezaei, N. Computational drug discovery and repurposing for the treatment of COVID-19: a systematic review. Bioorg. Chem. 106, 104490 (2021).

385. Nukoolkarn, V., Lee, V. S., Malaisree, M., Aruksakulwong, O. \& Hannongbua, S. Molecular dynamic simulations analysis of ritronavir and lopinavir as SARS-CoV 3CLpro inhibitors. J. Theor. Biol. 254, 861-867 (2008).

386. Law, W. Y., Asaruddin, M. R., Bhawani, S. A. \& Mohamad, S. Pharmacophore modelling of vanillin derivatives, favipiravir, chloroquine, hydroxychloroquine, monolaurin and tetrodotoxin as $\mathrm{M}(\mathrm{Pro})$ inhibitors of severe acute respiratory syndrome coronavirus-2 (SARS-CoV-2). BMC Res. Notes 13, 527 (2020).

387. Sundar, S. et al. Screening of FDA-approved compound library identifies potential small-molecule inhibitors of SARS-CoV-2 non-structural proteins NSP1 NSP4, NSP6 and NSP13: molecular modeling and molecular dynamics studies. $J$ Proteins Proteom. https://doi.org/10.1007/s42485-021-00067-w (2021).

388. Vicenti, I., Zazzi, M. \& Saladini, F. SARS-CoV-2 RNA-dependent RNA polymerase as a therapeutic target for COVID-19. Expert. Opin. Ther. Pat. 31, 325-337 (2021).

389. Hecel, A. et al. Zinc(II)-the overlooked Éminence Grise of Chloroquine's fight against COVID-19? Pharmaceuticals 13, 228 (2020).

390. Narayanan, N. \& Nair, D. T. Vitamin B12 may inhibit RNA-dependent-RNA polymerase activity of nsp12 from the SARS-CoV-2 virus. IUBMB Life 72, 2112-2120 (2020)

391. Elfiky, A. A. SARS-CoV-2 RNA dependent RNA polymerase (RdRp) targeting: an in silico perspective. J. Biomol. Struct. Dyn. 39, 3204-3212 (2021)

392. Durojaiye, A. B., Clarke, J.-R. D., Stamatiades, G. A. \& Wang, C. Repurposing cefuroxime for treatment of COVID-19: a scoping review of in silico studies. J. Biomol. Struct. Dyn. 39, 4547-4554 (2021).

393. Lo, M. K. et al. Broad-spectrum in vitro antiviral activity of ODBG-P-RVn: an orally-available, lipid-modified monophosphate prodrug of remdesivir parent nucleoside (GS-441524). Microbiol. Spectr. 9, e0153721 (2021).

394. Cox, R. M. et al. Oral prodrug of remdesivir parent GS-441524 is efficacious against SARS-CoV-2 in ferrets. Nat. Commun. 12, 6415 (2021).

395. Spratt, A. N. et al. Coronavirus helicases: attractive and unique targets of antiviral drug-development and therapeutic patents. Expert. Opin. Ther. Pat. 31, 339-350 (2021).

396. Illian, D. N. et al. Potential compounds from several Indonesian plants to prevent SARS-CoV-2 infection: a mini-review of SARS-CoV-2 therapeutic targets. Heliyon 7, e06001 (2021).

397. Canal, B. et al. Identifying SARS-CoV-2 antiviral compounds by screening for small molecule inhibitors of nsp15 eSome essential mutations mentioned abovendoribonuclease. Biochem. J. 478, 2465-2479 (2021).

398. Ortiz-Alcantara, J., Bhardwaj, K., Palaninathan, S. \& Et, A. Small molecule inhibitors of the SARS-CoV Nsp15 endoribonuclease. Virus Adapt Treat. 2, 125-133 (2010).
399. Malik, A. et al. In silico screening of phytochemical compounds and FDA drugs as potential inhibitors for NSP16/10 $5^{\prime}$ methyl transferase activity. J. Biomol. Struct. Dyn. https://doi.org/10.1080/07391102.2021.2005680 (2021).

400. Khan, R. J. et al. Targeting SARS-CoV-2: a systematic drug repurposing approach to identify promising inhibitors against 3C-like proteinase and 2'-O-ribose methyltransferase. J. Biomol. Struct. Dyn. 39, 2679-2692 (2021).

401. Sukumaran, V. et al. Telmisartan acts through the modulation of ACE-2/ANG $1-7 /$ mas receptor in rats with dilated cardiomyopathy induced by experimental autoimmune myocarditis. Life Sci. 90, 289-300 (2012).

402. Wang, N. et al. Chloroquine and hydroxychloroquine as ACE2 blockers to inhibit viropexis of 2019-nCoV Spike pseudotyped virus. Phytomedicine 79, 153333 (2020).

403. Valenzuela, R. et al. Interactions between ibuprofen, ACE2, renin-angiotensin system, and spike protein in the lung. Implications for COVID-19. Clin. TransI. Med. 11, e371 (2021).

404. Ciulla, M. M. SARS-CoV-2 downregulation of ACE2 and pleiotropic effects of ACEIs/ARBs. Hypertens. Res. 43, 985-986 (2020).

405. Khan, A. et al. A pilot clinical trial of recombinant human angiotensin-converting enzyme 2 in acute respiratory distress syndrome. Crit. Care 21, 234 (2017).

406. Ou, X. Y. et al. Characterization of spike glycoprotein of SARS-CoV-2 on virus entry and its immune cross-reactivity with SARS-CoV. Nat. Commun. 11, 1620 (2020).

407. Shah, P. P. et al. A small-molecule oxocarbazate inhibitor of human cathepsin L blocks severe acute respiratory syndrome and ebola pseudotype virus infection into human embryonic kidney 293 T cells. Mol. Pharmacol. 78, 319-324 (2010)

408. Cheng, Y. W. et al. Furin inhibitors block SARS-CoV-2 spike protein cleavage to suppress virus production and cytopathic effects. Cell Rep. 33, 108254 (2020).

409. Bahbouhi, B., Bendjennat, M., Guétard, D., Seidah, N. G. \& Bahraoui, E. Effect of alpha-1 antitrypsin Portland variant (alpha 1-PDX) on HIV-1 replication. Biochem. J. 352, 91-98 (2000)

410. Li, K., Meyerholz, D. K., Bartlett, J. A. \& McCray, P. B. Jr The TMPRSS2 inhibitor nafamostat reduces SARS-CoV-2 pulmonary infection in mouse models of COVID-19. mBio 12, e0097021 (2021).

411. Hoffmann, M. et al. Camostat mesylate inhibits SARS-CoV-2 activation by TMPRSS2-related proteases and its metabolite GBPA exerts antiviral activity. EBioMedicine 65, 103255 (2021).

412. Ansarin, K. et al. Effect of bromhexine on clinical outcomes and mortality in COVID-19 patients: a randomized clinical trial. Biolmpacts 10, 209-215 (2020).

413. Shen, L. W. et al. Inhibition of influenza A virus propagation by benzoselenoxanthenes stabilizing TMPRSS2 gene G-quadruplex and hence down-regulating TMPRSS2 expression. Sci. Rep. 10, 7635-7635 (2020).

414. Meyer, D. et al. Identification of the first synthetic inhibitors of the type II transmembrane serine protease TMPRSS2 suitable for inhibition of influenza virus activation. Biochem. J. 452, 331-343 (2013).

415. Elmezayen, A. D., Al-Obaidi, A., Sahin, A. T. \& Yelekçi, K. Drug repurposing for coronavirus (COVID-19): in silico screening of known drugs against coronavirus 3CL hydrolase and protease enzymes. J. Biomol. Struct. Dyn. 39, 2980-2992 (2021).

416. Lemes, R. M. R. et al. 17ß-estradiol reduces SARS-CoV-2 infection in vitro. Physiol. Rep. 9, e14707 (2021).

417. Leach, D. A. et al. The antiandrogen enzalutamide downregulates TMPRSS2 and reduces cellular entry of SARS-CoV-2 in human lung cells. Nat. Commun. 12, 4068 (2021).

418. Drożdżal, S. et al. FDA approved drugs with pharmacotherapeutic potential for SARS-CoV-2 (COVID-19) therapy. Drug Resist. Updat 53, 100719 (2020).

419. Ulrich, H. \& Pillat, M. M. CD147 as a target for COVID-19 treatment: suggested effects of azithromycin and stem cell engagement. Stem Cell Rev. Rep. 16, 434-440 (2020)

420. Yates, P. A. et al. Doxycycline treatment of high-risk COVID-19-positive patients with comorbid pulmonary disease. Ther. Adv. Respir. Dis. 14, 1753466620951053 (2020).

421. Gelman, R., Bayatra, A., Kessler, A., Schwartz, A. \& Ilan, Y. Targeting SARS-CoV-2 receptors as a means for reducing infectivity and improving antiviral and immune response: an algorithm-based method for overcoming resistance to antiviral agents. Emerg. Microbes Infect. 9, 1397-1406 (2020).

422. Mantlo, E., Bukreyeva, N., Maruyama, J., Paessler, S. \& Huang, C. Antiviral activities of type I interferons to SARS-CoV-2 infection. Antivir. Res. 179, 104811 (2020).

423. Chiscano-Camón, L., Ruiz-Rodriguez, J. C., Ruiz-Sanmartin, A., Roca, O. \& Ferrer, R. Vitamin $C$ levels in patients with SARS-CoV-2-associated acute respiratory distress syndrome. Crit. Care 24, 522 (2020).

424. Pelaia, C. et al. Therapeutic role of Tocilizumab in SARS-CoV-2-induced cytokine storm: rationale and current evidence. Int. J. Mol. Sci. 22, 3059 (2021). 
Structural biology of SARS-CoV-2: open the door for novel therapies Yan et al.

(c) Open Access This article is licensed under a Creative Commons Attribution 4.0 International License, which permits use, sharing, adaptation, distribution and reproduction in any medium or format, as long as you give appropriate credit to the original author(s) and the source, provide a link to the Creative Commons license, and indicate if changes were made. The images or other third party material in this article are included in the article's Creative Commons license, unless indicated otherwise in a credit line to the material. If material is not included in the article's Creative Commons license and your intended use is not permitted by statutory regulation or exceeds the permitted use, you will need to obtain permission directly from the copyright holder. To view a copy of this license, visit http://creativecommons. org/licenses/by/4.0/.

(c) The Author(s) 2022 